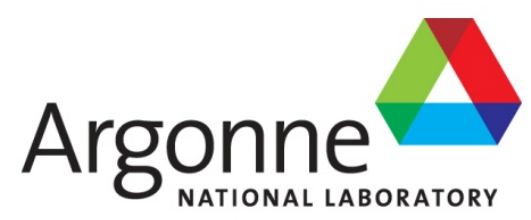

ANL/DIS-10-15

NATIONAL LABORATORY

\title{
Analysis of Combined Hydrogen, Heat, and Power as a Bridge to a Hydrogen Transition
}

Decision and Information Sciences Division 


\section{About Argonne National Laboratory}

Argonne is a U.S. Department of Energy laboratory managed by UChicago Argonne, LLC under contract DE-AC02-06CH11357. The Laboratory's main facility is outside Chicago,

at 9700 South Cass Avenue, Argonne, Illinois 60439. For information about Argonne

and its pioneering science and technology programs, see www.anl.gov.

\section{Availability of This Report}

This report is available, at no cost, at http://www.osti.gov/bridge. It is also available on paper to the U.S. Department of Energy and its contractors, for a processing fee, from:

U.S. Department of Energy

Office of Scientific and Technical Information

P.O. Box 62

Oak Ridge, TN 37831-0062

phone (865) 576-8401

fax (865) 576-5728

reports@adonis.osti.gov

\section{Disclaimer}

This report was prepared as an account of work sponsored by an agency of the United States Government. Neither the United States Government nor any agency thereof, nor UChicago Argonne, LLC, nor any of their employees or officers, makes any warranty, express or implied, or assumes any legal liability or responsibility for the accuracy, completeness, or usefulness of any information, apparatus, product, or process disclosed, or represents that its use would not infringe privately owned rights. Reference herein to any specific commercial product, process, or service by trade name, trademark, manufacturer, or otherwise, does not necessarily constitute or imply its endorsement, recommendation, or favoring by the United States Government or any agency thereof. The views and opinions of document authors expressed herein do not necessarily state or reflect those of the United States Government or any agency thereof, Argonne National Laboratory, or UChicago Argonne, LLC. 


\section{Analysis of Combined Hydrogen, Heat, and Power as a Bridge to a Hydrogen Transition}

M.R. Mahalik and C.H. Stephan

Decision and Information Sciences Division, Argonne National Laboratory

December 2010 
Analysis of CHHP as a Bridge to a Hydrogen Transition

This page intentionally blank 


\section{Contents}

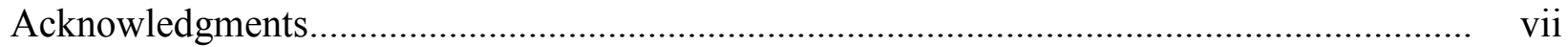

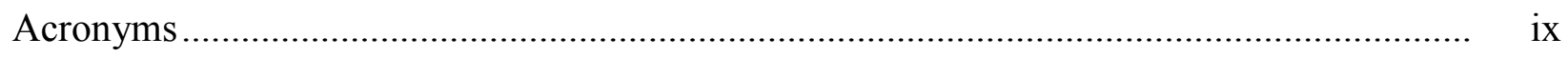

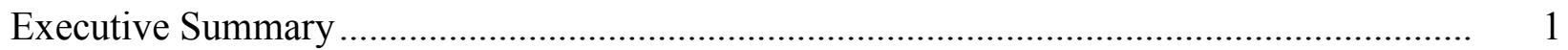

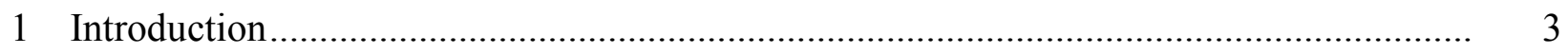

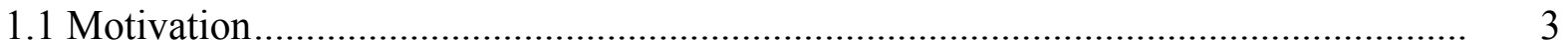

1.2 Assumptions/Scope..........................................................................................

2 Background of the Model .............................................................................................. 5

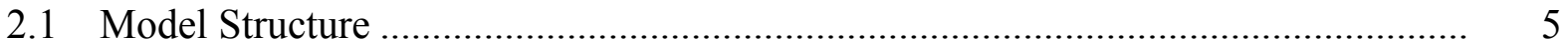

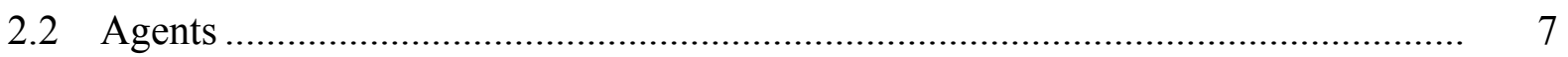

2.2.1 Driver Agents .......................................................................................... 7

2.2.2 Investor Agents...................................................................................... 9

2.3 Hydrogen Fueling Stations ................................................................................. 11

3 Improvements to the Base Model ............................................................................ 13

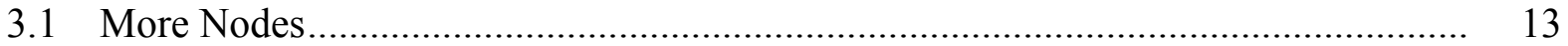

3.2 Calibration of Driver Agent Trip Lengths to NHTS Traffic Data ............................ 14

3.3 Driver Agent "Multiple Personalities"................................................................... 14

3.4 Driver Agent "Green" Attributes ............................................................................. 15

3.5 Recalibration of the Algorithm to Predict Hydrogen Sales at an Unoccupied Site... 15

3.6 Improved Investor Algorithm .............................................................................. 16

4 Modifications to the Model to Incorporate HDFs.................................................................. 17

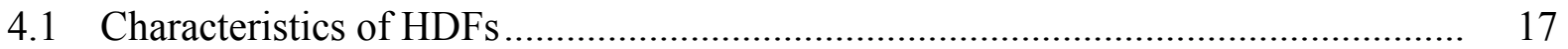

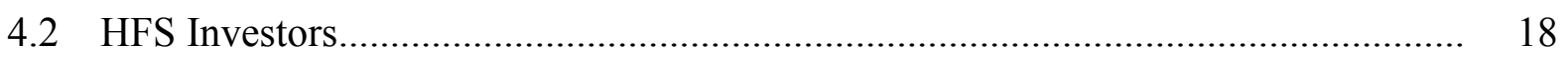

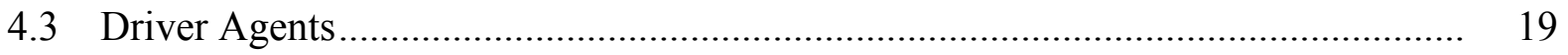

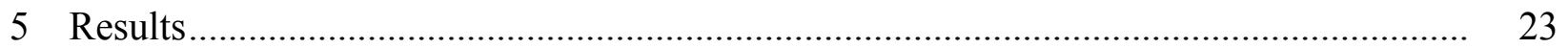

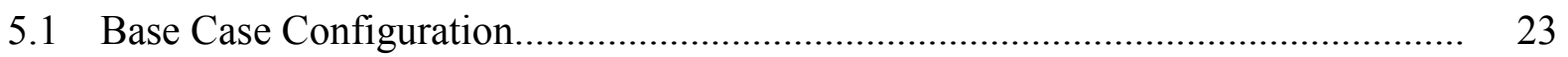

5.2 Base Case Results ......................................................................................... 24

5.2.1 Investor Variations ............................................................................ 26

5.2.2 Sensitivity to Agent Interactions ............................................................. 28

5.3 Introduction of HDFs ....................................................................................... 29

5.3.1 HDF Fuel Price.................................................................................... 30

5.3.2 "Intelligent" Placement of Fewer HDFs ...................................................... 32 
5.3.3 Increased Capacity of HDFs........................................................................... 34

5.3.4 Early Shutdown of HDFs ……………………...................................... 35

5.3.5 Effect of Drivers' Weighting of Worry and Inconvenience........................... 36

5.3.6 Vehicle Range ....................................................................................... 37

5.3.7 Driver Discounting of Past Experience .......................................................... 37

5.3.8 HFS Investor Returns ………………………....................................... 38

5.3.9 Geographic Results............................................................................... 40

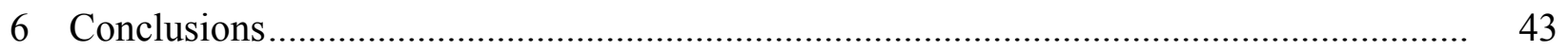

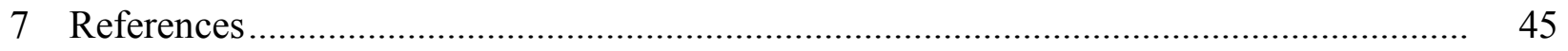

Appendix A: Fit of Driver Agent Trip Length Distribution to NHTS Data .......................... 47

Appendix B: Facilities Identified as Candidate Locations for CHHP Plants .......................... 55

\section{Figures}

1 Los Angeles, California, Metropolitan Area Showing Modeling Grid Structure and Major Expressways, as Represented in the Grid Format.................................................... 6

2 Population Densities in the Model Area ……................................................................ 7

3 Reaction of Different "Personalities" to the Percentage of HPVs Owned by their Neighbors, Coworkers, and the General Public ................................................................. $\quad 10$

4 Respondent Willingness to Pay Extra for a "Green” Technology Vehicle ....................... 15

5 Current Topology, Including Roadway Nodes and Candidate HDF Sites ......................... 18

6 HPV Fleet Penetration, Drivers Preferring HPVs, and Aggregate HFS Capacity in the Base Case ....................................................................................................... 25

7 Geographic Distribution of HPVs in the Base Case ……................................................ 26

8 and 9 HPV Fleet Penetration and Average HFS Capacity Factor When HFS Rollout Is under the Control of the Investor Algorithm and a Simple Heuristic ............................ 27

10 Fleet Penetration of HPVs under Various Hypothetical Scenarios ................................... 29

11 HPV Fleet Penetration under the Base Case and with HDFs Selling Hydroegn at Various Prices …………………………................................................................... 31

12 Sales for 74 HDFs Randomly Picked from the Total Population of 121.......................... 33 


\section{Figures (Cont.)}

13 Penetration of HPVs into the Fleet for (a) All 121 HDFs Brought On-line in the First Year and (b) Smaller Numbers of HDFs Brought On-line in Accordance with the Protocols

14 Effect of Increasing the Capacity of HDFs from 73 to $150 \mathrm{~kg} /$ day

15 Effect of Increasing the Capacity of HDFs from 73 to $150 \mathrm{~kg}$ /day with Expanded Scales for Early Years

16 Effect on HPV Penetration of Removing All HDFs at the End of Years 3 and 5

17 HPV Adoption Rate When Drivers Discount Past Experience More Heavily

18 Investor IRRs and NPVs under Scenarios of No HDFs and "Protocol 20" Rollout of HDFs

19 Number of Investor-built HFSs under the Two Scenarios in Figure 18

20 Difference in Household Hydrogen Fuel Consumption between a Case with and without HDFs

\section{Table}

1 Selected Base Case Parameter Values 
Analysis of CHHP as a Bridge to a Hydrogen Transition

This page intentionally blank 


\section{Acknowledgments}

This study was supported by the Fuel Cell Technologies Program (U.S. Department of Energy, Assistant Secretary for Energy Efficiency and Renewable Energy) under Contract Number DEAC02-06CH11357. We would like to thank Fred Joseck of the Fuel Cell Technologies Program for his support of this study. From Argonne National Laboratory, we thank Marianne Mintz and Guenter Conzelmann for valuable discussions, Luke VanLandegen for GIS support, and Mary Fitzpatrick for editorial support. Lastly we thank Darlene Steward and Michael Penev of the National Renewable Energy Laboratory for discussions of the Fuel Cell Power Model and Stephen Popiel and Scott Miller of Synovate Motoresearch for providing insights into consumer decision making. 
Analysis of CHHP as a Bridge to a Hydrogen Transition

This page intentionally blank 


\section{Acronyms}

\begin{tabular}{|c|c|}
\hline ABMS & agent- based modeling and simulation \\
\hline CHHP & combined hydrogen, heat, and power \\
\hline DOE & U.S. Department of Energy \\
\hline GPS & global positioning system \\
\hline $\begin{array}{l}\mathrm{H} 2 \mathrm{CAS} \\
\mathrm{HDF}^{1}\end{array}$ & $\begin{array}{l}\text { Hydrogen Complex Adaptive System (simulation model) } \\
\text { hydrogen dispensing facility (limited-service small-scale hydrogen dispenser } \\
\text { associated with a CHHP plant) }\end{array}$ \\
\hline $\begin{array}{l}\mathrm{HFS}^{1} \\
\mathrm{HPV}^{1}\end{array}$ & $\begin{array}{l}\text { hydrogen fueling station (full-service, large-scale hydrogen refueling facility) } \\
\text { hydrogen-powered vehicle }\end{array}$ \\
\hline IRR & internal rate of return \\
\hline MY & model year \\
\hline $\begin{array}{l}\text { NHTS } \\
\text { NPV } \\
\text { NREL }\end{array}$ & $\begin{array}{l}\text { National Household Travel Survey } \\
\text { net present value } \\
\text { National Renewable Energy Laboratory }\end{array}$ \\
\hline $\begin{array}{l}\text { OEM } \\
\text { ORNL }\end{array}$ & $\begin{array}{l}\text { original equipment manufacturer } \\
\text { Oak Ridge National Laboratory }\end{array}$ \\
\hline $\begin{array}{l}\text { RCF } \\
\text { ROI }\end{array}$ & $\begin{array}{l}\text { RCF Economic and Financial Consulting, Inc. } \\
\text { return on investment }\end{array}$ \\
\hline
\end{tabular}

1 These terms have been adopted for clarity in this report and are not necessarily in common usage. 
Analysis of CHHP as a Bridge to a Hydrogen Transition

This page intentionally blank 


\section{Executive Summary}

Combined hydrogen, heat, and power (CHHP) technology is envisioned as a means to providing heat and electricity, generated on-site, to large end users, such as hospitals, hotels, and distribution centers, while simultaneously producing hydrogen as a by-product. The hydrogen can be stored for later conversion to electricity, used on-site (e.g., in forklifts), or dispensed to hydrogen-powered vehicles. Argonne has developed a complex-adaptive-system model, H2CAS, to simulate how vehicles and infrastructure can evolve in a transition to hydrogen. This study applies the H2CAS model to examine how CHHP technology can be used to aid the transition to hydrogen. It does not attempt to predict the future or provide one forecast of system development. Rather, the purpose of the model is to understand how the system works.

The model uses a 50- by 100-mile rectangular grid of 1-square-mile cells centered on the Los Angeles metropolitan area. The major expressways are incorporated into the model, and local streets are considered to be ubiquitous, except where there are natural barriers. The model has two types of agents. Driver agents are characterized by a number of parameters: home and job locations, income, various types of "personalities" reflective of marketing distinctions (e.g., innovators, early adopters), willingness to spend extra money on "green" vehicles, etc. At the beginning of the simulations, almost all driver agents own conventional vehicles. They drive around the metropolitan area, commuting to and from work and traveling to various other destinations. As they do so, they observe the presence or absence of facilities selling hydrogen. If they find such facilities conveniently located along their routes, they are motivated to purchase a hydrogen-powered vehicle when it becomes time to replace their present vehicle. Conversely, if they find that they would be inconvenienced by having to purchase hydrogen earlier than necessary or if they become worried that they would run out of fuel before encountering a facility, their motivation to purchase a hydrogen-powered vehicle decreases. At vehicle purchase time, they weigh this experience, as well as other factors such as social influence by their peers, fuel cost, and capital cost of a hydrogen vehicle.

Investor agents build full-service hydrogen fueling stations (HFSs) at different locations along the highway network. They base their decision to build or not build a station on their (imperfect) estimates of the sales the station would immediately generate (based on hydrogen-powered vehicle traffic past the location and other factors), as well as the growth in hydrogen sales they could expect throughout their investment horizon. The interaction between driver and investor agents provides the basis for growth in both the number of hydrogen vehicles and number of hydrogen stations.

For the present report, we have added to this mix smaller, "bare-bones" hydrogen dispensing facilities (HDFs) of the type that owners of CHHP facilities could provide to the public. The locations of these stations were chosen to match existing facilities that might reasonably incorporate CHHP plants in the future. Unlike the larger commercial stations, these facilities are built according to exogenously supplied timetables, and no attempt has been made to model the financial basis for the facilities. Rather, our objective is to understand how the presence of these additional stations might facilitate the petroleum-to-hydrogen transition. 
We discuss a base case in which the HDFs are not present, and then investigate the effects of introducing HDFs in various numbers; according to different timetables; with various production capacities; and with hydrogen selling at prices above, equal to, and below the commercial stations' selling price. We conclude that HDFs can indeed be helpful in accelerating a petroleumto-hydrogen transition. Placed in areas where investors might not be willing to install large forprofit HFSs, HDFs can serve as a bridge until demand for hydrogen increases to the point where larger stations are viable. For drivers to use them, however, HDFs must sell hydrogen at a price competitive with that of the larger HFSs. Because the marginal cost of producing hydrogen is expected to be higher for small capacities, HDFs may be uncompetitive without (possibly substantial) subsidies or other means to allow them to sell hydrogen at a price below the actual cost to produce and distribute it. Fortunately, HDFs do not need to be subsidized for very long. In our model, installing HDFs only as the demand for hydrogen increased was effective; then closing down all HDFs after five years into the transition made virtually no difference to the resultant accelerated outcome. Even installing much smaller numbers of HDFs only in the second and third years of the simulation and then closing them at the end of the fifth year was helpful. Another important conclusion is that HDFs, selling hydrogen at a subsidized price if necessary to be competitive with HFSs, do not "steal" sales from these large-scale stations, at least over the long term. By allowing drivers to purchase hydrogen at places where HFSs are not available, HDFs increase the rate of adoption of hydrogen-powered vehicles so that private investors actually make more money over their investment horizon. 


\section{Introduction}

Combined hydrogen, heat, and power (CHHP) technology is envisioned as a means to providing heat and electricity, generated on-site, to large end users, such as hospitals, hotels, and distribution centers, while simultaneously producing hydrogen as a by-product. The hydrogen can be stored for later conversion to electricity, used on-site (e.g., in forklifts), or dispensed to hydrogen-powered vehicles (HPVs). ${ }^{2}$ Argonne has developed a complex-adaptive-system model, $\mathrm{H} 2 \mathrm{CAS}$, to simulate how vehicles and infrastructure can evolve in a transition to hydrogen. (See RCF Economic and Financial Consulting, Inc., and Argonne National Laboratory 2010 [1] and related publications $[2,3,4]$.) This study applies the H2CAS model to examine how CHHP technology can be used to aid the transition to hydrogen.

\subsection{Motivation}

Depending on how much hydrogen can be produced from the CHHP plant and how much is needed for internal use, it may be desirable to sell the excess hydrogen to the public to fuel HPVs. Legislation aimed at accelerating the use of HPVs may also require CHHP facilities to provide hydrogen to motorists. This possibility raises a number of important issues, such as:

- How might the presence of CHHP facilities affect the overall transition to hydrogen?

- Could the presence of CHHP facilities early in the transition help spur demand for HPVs and, in turn, private investment in dedicated HFSs?

- How can consumer demand for hydrogen at a candidate CHHP location be estimated?

- Assuming the fuel-dispensing portion of the CHHP facility will compete with dedicated HFSs placed in perhaps more convenient locations, what discounts or other motivators may be needed to induce HPV drivers to patronize CHHP facilities?

- Some locations may be good candidates for CHHP, but less than ideal for HPV drivers. What incentives will be necessary to induce drivers to make a detour in order to patronize a CHHP facility? Conversely, would some locations be particularly attractive to drivers and, thus, better candidates for CHHP?

- How will carbon taxes/credits and infrastructure subsidies affect the selling price of the hydrogen?

${ }^{2}$ While the leading candidate for converting hydrogen to motive power in vehicles is the fuel cell, we use the more general term "hydrogen-powered vehicle" to emphasize that the model is applicable to personal highway vehicles using hydrogen of any type, including ones using hydrogen internal combustion engines. 
- A small CHHP facility may run the risk of running out of hydrogen, with consequent inconvenience and worry to drivers. How successful might these facilities be depending on the drivers' levels of aversion to such uncertainty?

- How could CHHPs fit into a competitive marketplace, in which stations focusing only on selling hydrogen fuel to consumers also exist?

- How does the importance of CHHPs as a bridge depend upon the refueling range of the available HPVs?

- If CHHPs do aid in the transition and a network of dedicated refueling stations does emerge, is there some point at which selling of hydrogen from CHHPs is no longer necessary to ensure the continued success of the transition?

An agent-based model, incorporating a diverse set of agents and operating in a well-specified geographical environment, is well-suited to address such questions.

\subsection{Assumptions/Scope}

It is not our objective in this study to perform an economic analysis of the operation of CHHP plants or determine whether the construction of a CHHP plant at a particular facility, or even in general, is warranted. Indeed, the decision as to whether a CHHP plant should sell hydrogen to motorists may be driven by factors other than financial ones, such as legislative requirements. The National Renewable Energy Laboratory (NREL) has already developed models to analyze the financial implications of CHHP systems using molten carbonate and phosphoric acid fuel cells [5]. The strength of our model, and thus the focus of our analysis, is to examine how the presence of such facilities could affect the transition, assuming that the proper conditions (in terms of technology, economy, and/or policy) exist to make the deployment of CHHPs - and their selling of hydrogen as an ancillary service - feasible. We assume that drivers will access the fuel via a small hydrogen dispensing facility (HDF), which may be physically separate and/or fenced off from the CHHP plant and the larger facility that it serves, and that the proper safety regulations, zoning requirements, etc. will be in place to allow such an arrangement.

It is important to note that this analysis does not aim to predict the future. Rather than to provide one forecast of system development, the purpose of our model is to understand how the system works. Our model has a large number of parameters ranging from the basic attributes of technology (e.g., tank capacity and fuel efficiency of the vehicles, production capacity of fueling stations) to the behavior and attitudes of the drivers (e.g., how to choose a refueling station, valuation of fuel savings relative to the sticker price of a vehicle), to strategies employed by the infrastructure investors (technique of growth anticipation, method of capital financing, minimum acceptable return on investment). Tuning each parameter to match actual future conditions, as well as accurately characterizing each consumer, infrastructure investor, and vehicle manufacturer is clearly an impossible task. The utility of the model lies in the ability it gives the user to change these parameters over a range of possible values and examine the tradeoffs that one scenario has with others with regard to the success of the transition. 


\section{Background of the Model}

The H2CAS model was developed during 2005-2009 for the Analysis of the Hydrogen Production and Delivery Infrastructure as a Complex Adaptive System, a project conducted for the Hydrogen, Fuel Cells and Infrastructure Technologies Program of the U.S. Department of Energy's (DOE's) Office of Energy Efficiency and Renewable Energy. The project built on work begun at Ford Motor Company $[6,7]$ and was a collaborative effort consisting of members from Argonne National Laboratory, RCF Economic and Financial Consulting, Inc., Ford Motor Company, BP, and others.

The purpose of the project was to analyze market and policy influences on the construction of infrastructure necessary to support a hydrogen transportation system. The goal of the model was not to provide a single forecast of how the hydrogen transportation system will develop but to explore (1) how different policies could affect the future of the hydrogen economy, (2) the sensitivity of the growth of the hydrogen economy to circumstances not controlled by policy, and (3) the sensitivity to differences in the behavioral responses of the actors compared with those in a benchmark scenario. A report on the development of this base model was released in 2010 [1]. Other related publications include papers focusing on driver logic [2, 3] and investor logic [4].

The methodology used in the project was agent-based modeling and simulation (ABMS). From its beginning in $1971[8,9]$, agent-based modeling has become a common simulation tool and today encompasses a wide variety of applications. ABMS can be especially helpful for a complex problem like the one addressed in our study that involves a diversity of players. Such modeling uses many "agents" (typically thousands) that are heterogeneous, autonomous actors and decision makers. Agents possess various and differing characteristics and interact with one another according to rules specified in a computer simulation. Agents have varying degrees of foresight, are generally designed to be imperfect, and can learn from their mistakes. Other agentbased models of a transition from petroleum to hydrogen transportation have been developed by Schwoon [10] and Keles [11], both of whom model a transition in Germany.

\subsection{Model Structure}

In our simulation, agents are of three types: potential buyers of hydrogen vehicles (drivers), potential investors in hydrogen fueling infrastructure, and vehicle original equipment manufacturers (OEMs). Drivers and investors interact with each other and with one another over a series of simulated time steps spanning a number of years, typically 20 . The characteristics of the individual drivers, along with their experiences on the road with the existing and growing hydrogen infrastructure, lead them to purchase either hydrogen or conventional vehicles and the corresponding fuel. Investor agents observe the changing demand for hydrogen; develop an expectation of future consumer demand and future competitive supply expansion; and, on the basis of this information, build the fueling infrastructure required to achieve individual financial goals. The OEM agent may limit the supply of HPVs in the early years as a result of manufacturing constraints. 
A number of improvements have been made to the model since the previous report. They are mentioned in their appropriate contexts in this section and described more fully in Section 3.

Our model region is a 100- by 50-mile rectangular area centered on the Los Angeles, California, metropolitan area and divided into 5,000 square cells (Figure 1). The roads on which agents travel include major highways and arterials (shown as green line segments in Figure 1) and "ubiquitous" local roads passing through every cell except where there are natural barriers. This approach is similar to one being used by Daimler AG in real-world tests to help place future HFSs in the most efficient locations [12]. Potential locations for hydrogen stations are located at the intersections of major roads and at other points between intersections. For this analysis, the number of such nodes is substantially increased (from 168 to 557) to provide more realism. The original 168 nodes are indicated by the yellow cells in Figure 1 (another figure which follows later shows the new configuration). In addition, there is a 25-mile-wide buffer zone surrounding this region; agents do not live in this zone but may travel into it. The buffer zone addresses the border condition of our environment: without the buffer zone, drivers would traverse border areas only as destinations rather than also as a means to reach areas beyond, so observed demand for fuel in those areas would be artificially low.

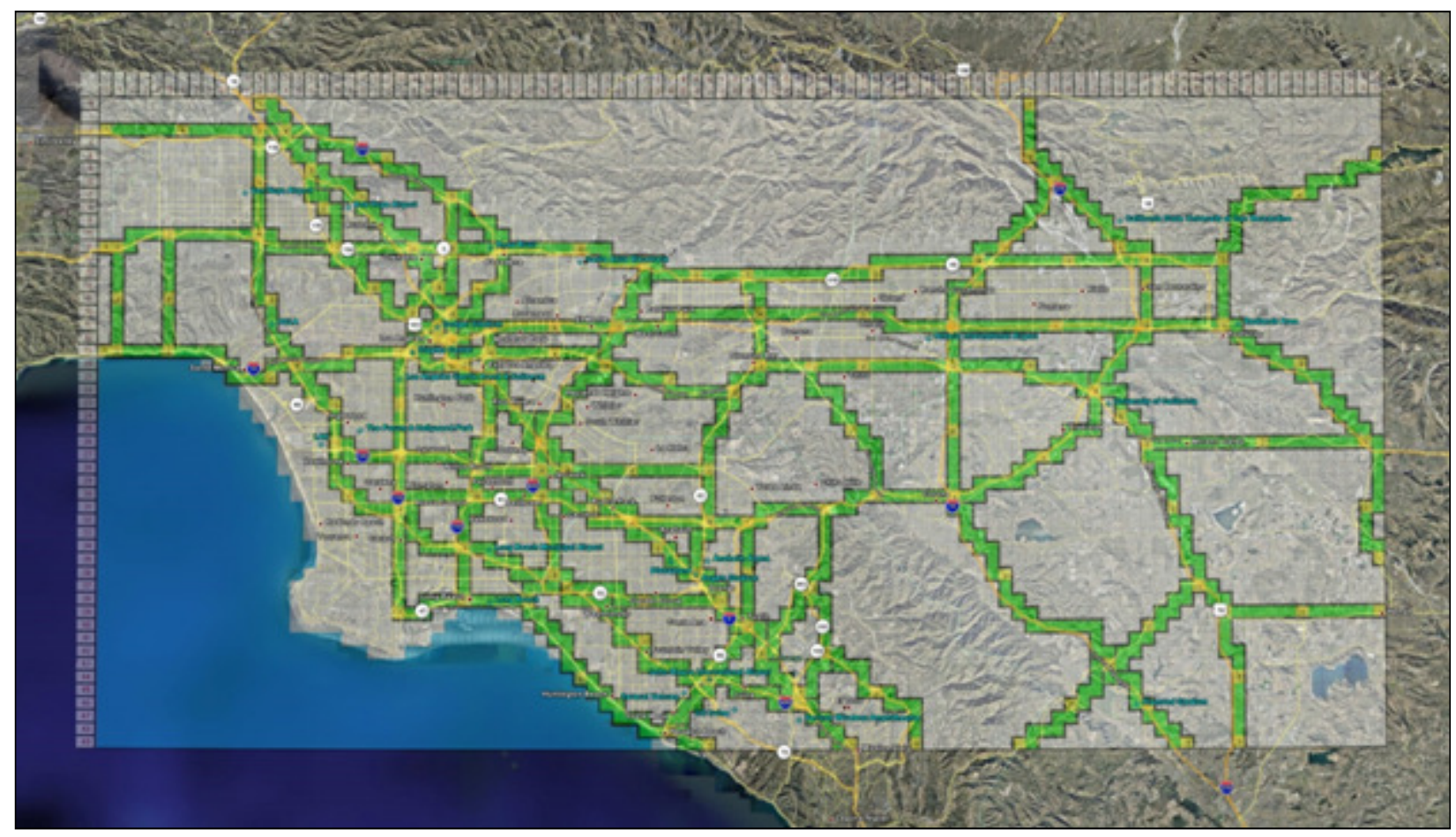

Figure 1: Los Angeles, California, Metropolitan Area Showing Modeling Grid Structure (not including buffer zone) and Major Expressways, as Represented in the Grid Format 


\subsection{Agents}

The model incorporates two types of agents: drivers and investors. Vehicle OEMs are limited to determining the supply of HPVs in the early years, and are controlled exogenously. The actions of the other two types are determined fully endogenously, as discussed briefly below. The model is quite complex, and the descriptions provided here are sufficient only to provide a general understanding of how it works. For more detailed descriptions, the reader should consult the 2010 RCF Economic and Financial Consulting, Inc., and Argonne National Laboratory report [1].

\subsubsection{Driver Agents}

Driver agents are assigned income levels on the basis of available demographic data for the Los Angeles area. High-, middle-, and low-income driver agents are randomly located predominantly, but not exclusively, in neighborhoods of corresponding income level and are distributed such that the overall agent population density scales to the region's actual population density (Figure 2). Similarly, agents are randomly, but preferentially, assigned to "jobs" in locations relatively near their homes and with average salary levels that preferentially match their incomes.

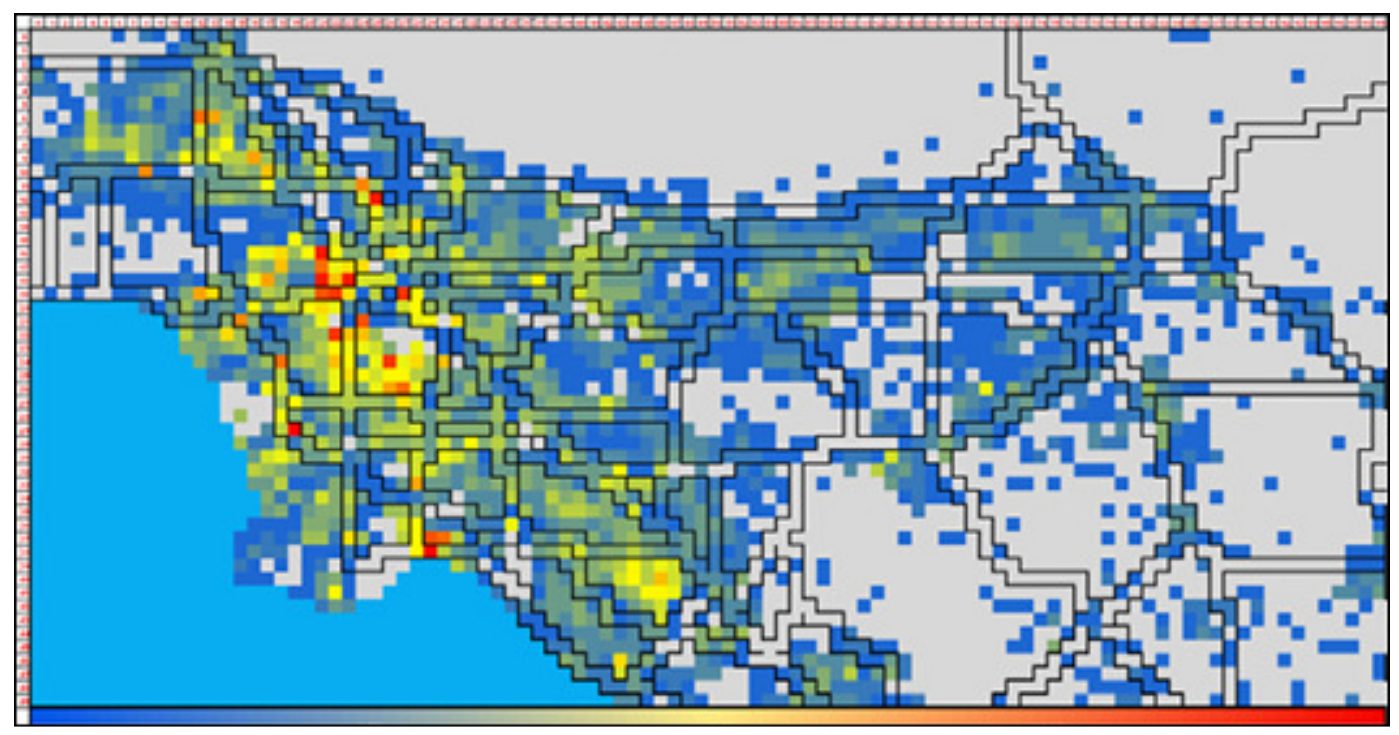

Figure 2: Population Densities in the Model Area (households per square mile, blue=low, red=high)

At the beginning of the simulation, almost all driver agents own conventional, petroleumpowered vehicles and only a few "seed" HFSs exist. In the course of a simulation, driver agents drive to and from work and to various destinations in the model region and buffer zone and note their exposure to the hydrogen fueling infrastructure regardless of what type of vehicle they own. 
The distances to these destinations have been recalibrated to provide better agreement with real driver behavior, as described in Section 3.

In this simulation, hydrogen stations (HFSs and HDFs) are represented on a one-to-one basis that is, each "real" station has a corresponding station in the simulation. Ideally, driver agents and vehicles would be represented on a one-to-one basis as well. However, with a population of roughly 7.6 million drivers in the Los Angeles area, one-to-one representation becomes impossible in terms of both computer resources and run times. To capture the diversity of this population with a practical number of driver agents (7,600, or roughly a 1000:1 ratio), we introduced the concept of "millicars," wherein each agent owns not one, but a fleet of 1000 millicars of varying ages and types (conventional or hydrogen powered). The millicars have different age distributions depending upon agent income level, calculated such that the aggregate distribution matches that of real car ages [13]. A real driver replaces a vehicle on average only once every 14 years, but with the millicar concept each agent replaces some portion of his fleet, depending on his income level, every year. In this way all agents can react quickly to changing conditions.

Thus, when it comes time each year to purchase new millicars, the agent weighs the pluses and minuses he sees of owning hydrogen versus conventional technology. ${ }^{3} \mathrm{New}$ car buyers have a choice of whether to purchase HPVs or conventional vehicles, while used car buyers are restricted in their choice to what is available on the market. Each driver agent calculates his personal HPV "utility function," which incorporates the incentives and disincentives he encountered in his driving experience, his attitudes toward new technology, and the social influence of the purchasing behavior of other agents. If it is positive, he buys HPVs if they are available; if negative, he buys conventional vehicles if they are available. The factors included in the utility function include the following:

1. Sticker Price: The fixed difference in purchase price between an HPV and a comparable conventional vehicle, which may include tax credits or other policy incentives. Sticker price is an exogenous parameter that can change during the course of the simulation.

2. Price Drop: A decrease in the purchase price of an HPV as a result of economies of scale in the manufacturing process. This value changes endogenously based on the number of hydrogen vehicles being sold.

3. Operational Savings: The difference in price, per mile traveled, of operating an HPV compared to a conventional one. The average number of miles traveled per year per agent is 12,000 , although individual agents may drive more or less depending upon the age distribution of their vehicles and random factors. (Newer vehicles are driven more miles per year than older ones [13]).

\footnotetext{
3 Because our agents do not have gender attributes, they should more properly be referred to as "it." However for convenience, we refer to them in this report as "he (him/his)."
} 
4. Inconvenience: A disincentive representing the lack of convenient fueling stations. There are four types of inconvenience events that the driver of an HPV can experience: (1) the inability to make a desired trip at all using an HPV, (2) having to undertake a special trip solely for the purpose of refueling, (3) the need to purchase fuel earlier than desired, and (4) having to wait in line to refuel at a crowded station.

5. Worry: A disincentive representing concern about running out of fuel, which is triggered when a driver is unable to buy fuel before his fuel gauge falls below what he considers to be a comfortable threshold.

6. Personality/Influence: The effect of the individual driver agent's attitude toward new technology, along with the social influence resulting from interaction with others. "Personalities" describe how agents react to the ownership of new technology by their acquaintances and the general public. As shown in Figure 3, personalities range from "innovators", who want to be among the first to own the new technology and are actually demotivated to buy it if many others already have it; to "Luddites," who want to be assured that a new technology is acceptable by others having bought and tried it before they do. Percentages of each personality type in the general population were estimated in consultation with Synovate Motoresearch [14], a market research company specializing in the automotive market. Based on these percentages, individual drivers are randomly assigned different personality types. We expect innovators and early adopters to be more prevalent in the upper-income agents, if only because they are more able to afford the latest technology.

All of the above factors are cast in terms of present-value dollars to make them easily additive. Intangible factors, for example, the cost associated with the inconvenience of having to make a special hydrogen refueling trip based in part on the distance traveled, are assigned a dollar value as an input parameter. The agent sums up all such trips he made (or would have had to make if he had an HPV) over his driving experience, but weights recent experience more heavily. Clearly, it is a rare driver who does this math in the real world, but most drivers have some experience-based intuitive understanding of the inconvenience they can expect to suffer as a result of a scarcity of HFSs. It is this intuitive weighting of many different factors that we seek to simulate with a utility function.

\subsubsection{Investor Agents}

Investor agents attempt to assess the potential of each candidate location on the basis of measurements of traffic passing the location, historical sales records of existing stations at the same or nearby locations, and the effect of a new station in "cannibalizing" sales of existing stations that they own (although not, of course, those owned by competitors). Sales cannibalization is not necessarily bad, because HFSs (unlike HDFs) are supplied with expensive trucked-in hydrogen if necessary to avoid turning drivers away. Thus, an over-capacity station selling additional hydrogen at a loss will become more profitable if some of its sales are taken away by a new station. 


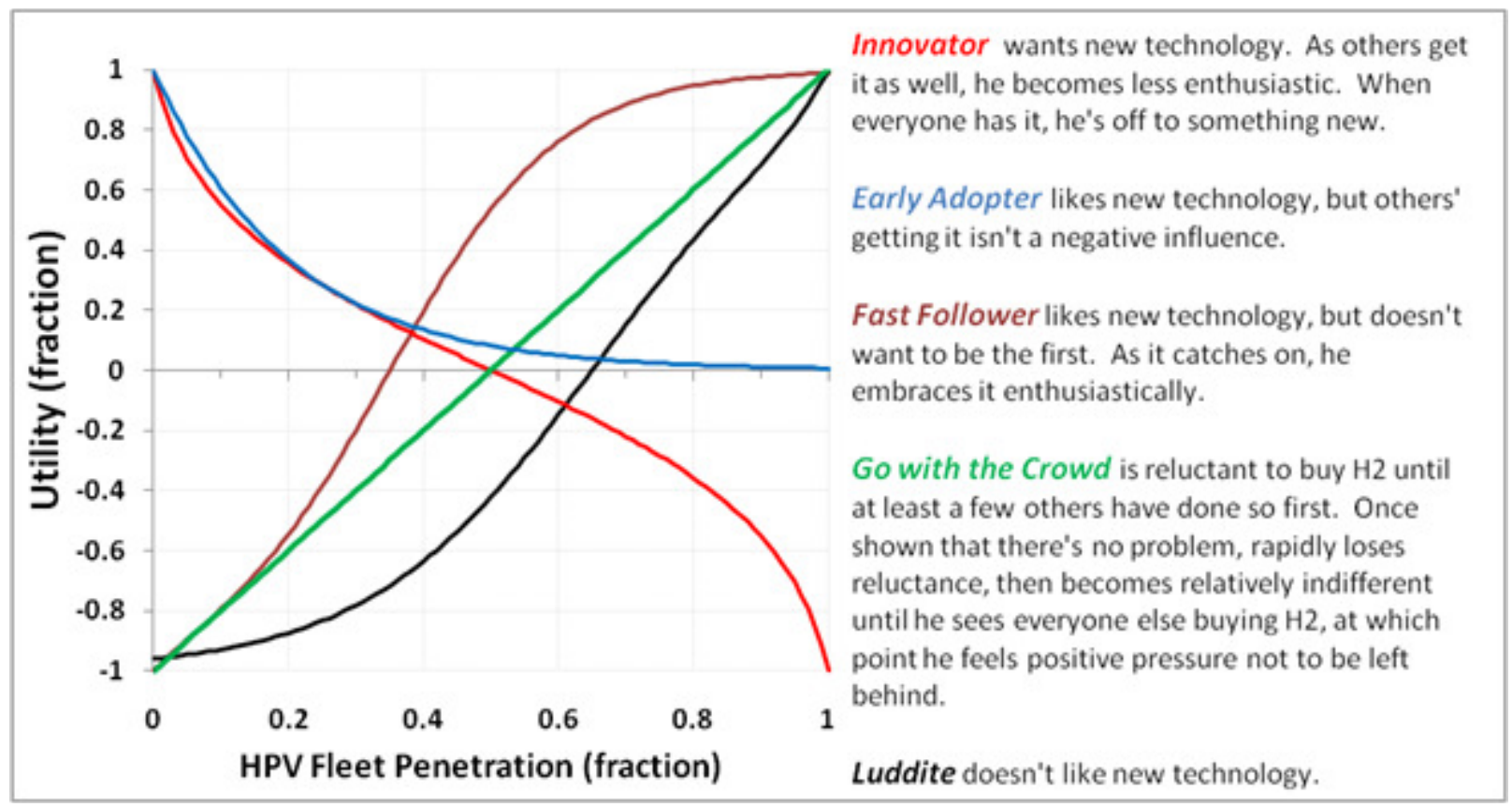

Figure 3: Reaction of Different "Personalities" to the Percentage of HPVs Owned by their Neighbors, Coworkers, and the General Public (The utility ranges from -1 [strongly against the new technology] to +1 [strongly in favor of it]. The utility is converted into dollars by an exogenously supplied multiplier. For example, a $\$ 1,000$ multiplier would mean that an agent with a -1 utility would buy the new technology only if it were $\$ 1,000$ cheaper than the conventional technology, all other things being equal. The multiplier can vary from one personality type to the next.)

Each investor has a time horizon (typically 10 years) over which he attempts to make a minimum return on his investment. Thus, he must attempt to predict sales not only in the first year of operation, but throughout this time period. In doing so, he must consider and anticipate a number of factors:

- As the popularity of HPVs increases, global demand for hydrogen will grow, and a new station can be expected to share in that demand and thus look forward to increased sales in future years. (Conversely, of course, popularity of hydrogen could decline, perhaps as a result of government policies or competition from alternative fuels.)

- While the siting of a station at a given location may temporarily discourage investors from building additional stations nearby, at some point increased hydrogen demand may be expected to lead competitors or the investor himself to site another station nearby, thereby cutting into sales from the first station.

The method by which an investor estimates the sales potential has been improved for this report and is described in Section 3. 
In addition to estimating the current sales potential at a given site, the investor must attempt to predict the course of sales over his investment horizon. Again, the method by which this is done has been improved and is described in Section 3.

A distinction is made between free-market and monopolistic investors. The free-market investor has no control over the actions of his competitors and can only try to anticipate those actions. A station justified only on the basis of anticipated growth in sales over the years can be expected to see such growth continue only until a competitor finds it profitable to locate another station, either in the same cell or a nearby one, and share in the sales. Contrary to this, a monopolistic investor's best strategy is to refrain from building nearby stations until the established station's sales reach capacity or even slightly exceed capacity. (Offsetting this strategy may be his expectation that placing a station in a nearby - not the same - cell may encourage overall growth in hydrogen sales, as mentioned above.) Finally, it should be noted that the fractional growth in hydrogen sales necessary to justify the establishment of an additional station at any given location decreases roughly as $1 / N$, where $N$ is the total number of stations in the region.

Having estimated expected sales over his investment time horizon, the investor computes the net present value of his cash flow over time according to a discount rate reflecting his desired return on investment (ROI). Components of the cash flow include equity financing, interest on debt, annual maintenance costs, hydrogen production (or purchase) costs, selling price, and the salvage value of the station (set to zero here in accordance with the assumption used in DOE's Hydrogen Analysis [H2A] Project that salvage value offsets decommissioning expense). If the value is positive, he does a further calculation to determine whether the value would be even greater if he waited a year before building in anticipation of further growth in hydrogen demand. If the second value is higher, he waits; if it is lower, his action depends on whether he is the sole investor or one of several competitors.

In the first case, he immediately builds the station. In the second case, each investor realizes that his competitors may also choose to build a station at the same location, with the result that there will be greater capacity than is justified by anticipated demand, and all will lose. To simulate what might happen in the "real world," we give each investor a $1 / \mathrm{N}$ chance of actually building the station, where $N$ is the number of competitors. The result of this process is the possibility of building 0 through $N$ stations at the site, with a single station having the highest probability. For example, in the case of four competitors, each assessing a given location as justifying the building of a single station, the random draw process may result in zero (31.6 percent probability), one (42.2 percent), two (21.1 percent), three (4.7 percent), or four ( 0.4 percent) stations actually built. The resulting potential inefficiency (too many or too few stations actually built) approximates what may happen in a real environment.

\subsection{Hydrogen Fueling Stations}

Primarily to reduce computation time, potential HFS locations are limited to roadway nodes along the major roadway network, although more than one station can be located at each node. The stations may produce hydrogen locally (through natural gas reforming) or purchase it from a centralized production facility. At this point, we have investigated only local production. Capital 
cost, production cost, selling price, production capacity, etc., are assigned exogenously and are the same for all stations. If the demand for hydrogen exceeds the capacity of the station or stations located at a given node, we assume that station owners will be forced to supplement their locally produced hydrogen with hydrogen purchased from a distributor and trucked to the location. Such hydrogen is expected to cost more than the locally produced variety, and the station owner may be forced to sell it at a loss. Trucked-in sales have a negative impact on driver agents as well, who suffer inconvenience from the expected additional waiting time to refuel, as discussed above. 


\section{Improvements to the Base Model}

In addition to changes in the model made specifically to incorporate HDFs, described later, a number of improvements were made to the base model to improve its performance and realism.

\subsection{More Nodes}

As described earlier, the topology over which the driver agents move consists of local roads that are ubiquitous, and major roads that cover just a portion of the model area. Driver agents choose their route between points using a shortest-path algorithm similar to that used by global positioning system (GPS) units, in which travel over the major roadways is faster than over the local roadways, and thus preferred by the drivers. ${ }^{4}$ Driver agents must enter, exit, and transfer between roads of the major roadway network via roadway nodes. Roadway nodes also serve as the candidate locations for hydrogen refueling stations.

The topology used in the previous version of the model was produced manually by overlaying a 1-square-mile grid on a map of the model area and then identifying which cells in the grid contained major roadways and the intersections of those roadways. Additional nodes were later added at the midpoints between most intersections, and each connecting segment had to be identified and measured so that the network could be accurately represented in the model. This process involved a considerable amount of manual work, discouraging any modification to the topology.

The more nodes in the topology, the greater its realism, allowing driver agents to more effectively use the major roadway network and giving investor agents a more diverse set of candidate locations to build refueling stations. To this end, we developed a new tool that makes the process of building an original topology or modifying an existing topology much easier. Using the mouse, a user of the tool draws the desired major roadways on a grid. Nodes can be added by clicking on particular cells, or the tool can automatically assign nodes according to several criteria (e.g., intersections, midpoints, every mile, every other mile). Once the user is finished drawing, the tool automatically identifies and measures each of the roadway segments and produces the data input files required to represent the topology in the model.

Using this tool, we modified the previous topology so that nodes are now present at least every other mile along each of the major roadways, a 3.6-fold increase over the previous configuration. The new configuration strikes a good balance between realism of the topology and the performance of the simulation; as the number of roadway nodes increase, so does the memory and computation time required to carry out a simulation.

\footnotetext{
4 Actually, to reduce computer running time, each driver agent is assigned a portfolio of 20 destinations (of which one is his job) during a pre-run, and the route calculations are made at that time. During each time step of the simulation, the agent draws from this portfolio to assemble a series of trips.
} 


\subsection{Calibration of Driver Agent Trip Lengths to NHTS Traffic Data}

For this study, we improved the calibration of agent driving behavior to real driver behavior as reflected in the National Household Travel Survey (NHTS) [15]. It is important not only that agent daily driving distances be realistic, but that the resulting maximum distances from their homes reflect actual behavior, since these distances determine how important local conditions are in influencing driver agent purchases. A driver who rarely ventures more than 15 miles from his house will not be affected by the presence or absence of HFSs 50 miles away. Fitting agent trips to actual data is complicated by the fact that driver agents make only two-segment trips (one out, one back), whereas NHTS data show daily trips ranging from one (presumably to an overnight stop) to 22 segments. Two-segment-only trips tend to underestimate the maximum distance from home of a one-segment trip, and overestimate the maximum distance for multisegment trips. The details of the fitting procedure are described in Appendix A.

\subsection{Driver Agent "Multiple Personalities"}

For this report, we introduce a new concept, similar in motivation to that of the millicars described in Section 2. This is the concept of driver agent "multiple personalities." Before, when agents purchased new millicars for their fleets, they bought either all HPVs or all conventional vehicles based on their utility functions. An agent's utility function includes terms reflecting his individual driving experience, universal factors such as fuel costs, and social influences from the agent's neighbors, coworkers, and the general population. The social influences are based on the fraction of late-model HPVs owned by the three cohorts and can be positive or negative, depending upon the agent's personality. Thus, the Innovator and Early Adopter personalities are influenced to buy HPVs to the extent that their social network influencers do not own them, while the Go with the Crowd and Luddite personalities are negatively influenced in such a situation and become positively influenced only when their influencers have at least 50 percent HPVs in their late-model fleets. Thus, even if an upper-income agent's utility function were only slightly in favor of hydrogen, all the 111 millicars bought new in a given year by the agent would be hydrogen-powered. Because each driver agent represents about 1,000 real drivers, the purchase represents 111 "real" HPVs. This is a large sale, at least in the beginning when HPV penetration is low, and could distort the local market. As a fix, we first tried a scheme in which driver agents purchased HPVs versus conventional vehicles in proportion to their utility. We encountered two problems with this approach. First, it was unclear how to calculate the range that is, at what dollar value of utility would an agent buy 100 percent of one type of vehicle or the other? Second, it meant that an agent who was, for example, 90 percent "against" hydrogen would still buy 10 percent HPVs, which did not seem reasonable.

With the introduction of "multiple personalities," each driver agent has all five personalities in proportions selected on the basis of discussions with Synovate Motoresearch [14]. Each subpersonality evaluates its own utility function. Thus, an upper-income agent's Early Adopter subpersonality, with a weight of 5 percent, may decide to purchase 5 percent $* 111=6$ hydrogen-powered millicars, whereas the agent's Luddite subpersonality, with a weight of 3 
percent, may buy 3 percent $* 111=3$ conventional millicars. In this way Early Adopters are able to buy HPVs without forcing the purchase of either 111 hydrogen vehicles or none.

\subsection{Driver Agent “Green” Attributes}

In addition to social-influence personalities, driver agents in the present simulations have been given "green" attributes based on market research studies carried out by Synovate. These studies asked respondents how much extra money they would be willing to pay to purchase a vehicle with environment-friendly attributes. On the basis of discussions with Synovate, we discounted these amounts by a factor of three to account for the discrepancy in what people say they will pay and what they are actually willing to pay. The adjusted data were fitted to an exponential curve (Figure 4), and each driver agent assigned a Green attribute based on a random draw from this distribution. (Interestingly, Synovate found that the stated willingness to pay depended mainly on the respondent's age, not on his or her income, so no attempt was made to correlate an agent's Green attribute with his income level.)

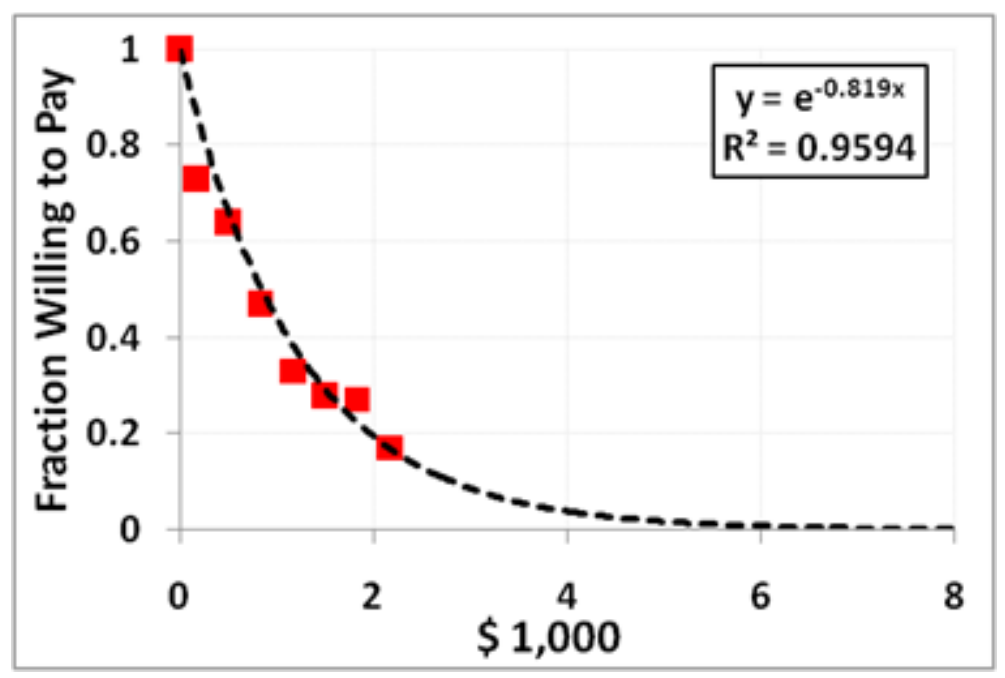

Figure 4: Respondent Willingness to Pay Extra for a "Green" Technology Vehicle (Source: Synovate Motoresearch [14])

\subsection{Recalibration of the Algorithm to Predict Hydrogen Sales at an Unoccupied Site}

An investor agent, deciding whether and when to build an HFS at an unoccupied site, must estimate the sales that such a station would generate. This is, of course, a complex decision based on many factors and one that real investors undoubtedly often do not make optimally. In the present simulation, many of the factors entering into a real investor's decision simply cannot be taken into account. Instead, investor agents estimate sales potential by counting the HPVs that drive by the location and, recognizing that the presence of nearby stations will reduce sales, use a relatively simple algorithm to correct the raw count data. This algorithm is based on sales of 
existing stations owned by the investor and specifically is a multiple linear regression of sales per traffic count as a function of the number of existing stations (investor or competitor owned) in each of up to three concentric rings around each station's location. This procedure is an attempt to give investor agents some insight into station viability beyond a simple traffic count, without providing them information that a real investor could not obtain. The free parameters were optimized by finding the values that produce the best $R^{2}$ correlation value in a number of preparatory simulations. Three rings with radii of 3, 6, and 15 miles were chosen as providing best estimates. Even so, correlation is not especially good (as might be expected with a simple algorithm), with $R^{2}$ coefficients ranging from the order of 0.4 and even lower up to 0.7 . Nevertheless, this may be as good as a "real" investor could do in such a situation.

\subsection{Improved Investor Algorithm}

In addition to estimating the first-year sales to be expected at a new location, the investor must attempt to predict the growth in sales over his investment horizon, typically 10 years. In the previous report, investor agents planning to build HFSs at new locations used an algorithm based on fitting previous sales at a given site (either real or estimated) to an S-shaped growth curve. This approach had the advantages that it was responsive to local conditions at the candidate site and that, theoretically, sales growth should follow such a curve (a Verhulst curve). However, the approach suffered from a paucity of sales data at local sites, especially in the early years, and hence the possibility of large random fluctuations in sales data and large errors in the fitted curves. Also, while overall hydrogen sales did indeed exhibit an S-shape behavior over the course of the simulations, sales at individual sites did not, because as sales increased, new stations were built, siphoning off sales from existing stations.

In the current simulations, predicted growth in sales at any site is based instead on a linear fit to the average growth in hydrogen sales per site over the previous time periods. While this method is not site-specific (i.e., a universal growth curve is obtained), it has the advantage that, especially in the critical early years of the transition, much more data are available on which to base the fit. Although only a linear fit, it was found to have better predictive capability than the Verhulst fit, again especially in the early years. (Higher power fits were also tried, but the results were usually less accurate in prediction than those of the linear fits.) Finally, sales data are taken on a quarterly rather than a yearly basis, again providing better predictive capability in the early years. Importantly, such data allow the investor agents to estimate growth endogenously, based on four quarters' experience, beginning in the fifth quarter when they first begin to build stations. Previously a first-year growth estimate had to be provided exogenously. 


\section{Modifications to the Model to Incorporate HDFs}

In addition to the improvements to the general model discussed in Section 3, many changes were made specifically to incorporate HDFs into the simulation.

\subsection{Characteristics of HDFs}

For the purpose of this discussion, an HDF has the following properties that distinguish it from a HFS:

- Normally smaller capacity (less than 100 to a few hundred $\mathrm{kg} /$ day of hydrogen production versus $1,500 \mathrm{~kg} /$ day for an HFS).

- Possibility of running out of hydrogen. Unlike HFSs, where (extra-cost) hydrogen is trucked in to supplement on-site production as necessary, a HDF sells only what it can produce. Consequently, drivers cannot count on its having fuel when they go there to refuel.

- Hydrogen selling price that may differ from that of HFSs (either higher or lower). All HFSs sell at a common price, and all HDFs sell at a common price.

Although the process is by no means simple, the decision of whether or not to build an HFS is concerned primarily with making a profit by matching hydrogen supply to a projected quarterly demand at a particular location. By contrast, the decision of whether or not it makes good economic sense to build a CHHP plant requires detailed knowledge of an individual facility's hourly energy loads and involves a complex set of factors and projections concerned not only with the characteristics of hydrogen but also with those of heat and electricity. The development of an algorithm to make such a decision is beyond the scope of this project. Therefore, HDFs are placed according to an exogenous schedule, rather than endogenously by an investment algorithm as HFSs are.

Facilities at which CHHP plants might be employed include large hospitals, hotels, shopping centers, universities, and distribution centers. The locations of such facilities in the Los Angeles area were identified using publicly available data and plotted onto the model topology. A list of the facilities identified is given in Appendix B.

As explained earlier, the structure of the model requires that refueling locations be positioned on the nodes of the major roadway network. The locations of some of the candidate CHHP sites identified did correspond with nodes in the existing topology, but others did not. For those that did not, a manual adjustment was made for each so that it would be associated with the roadway node closest to its actual location. Drivers wishing to use such an HDF would be required to make a detour of 1-5 miles from the associated node (the average detour length is 2 miles). The ability for drivers to detour for fuel is a new feature of the model and is described in Section 4.3. 
Figure 5 shows the current topology with candidate HDF sites. Green cells are major roadways, yellow cells are roadway nodes, and red cells are roadway nodes from which one or more candidate HDFs may be accessed.

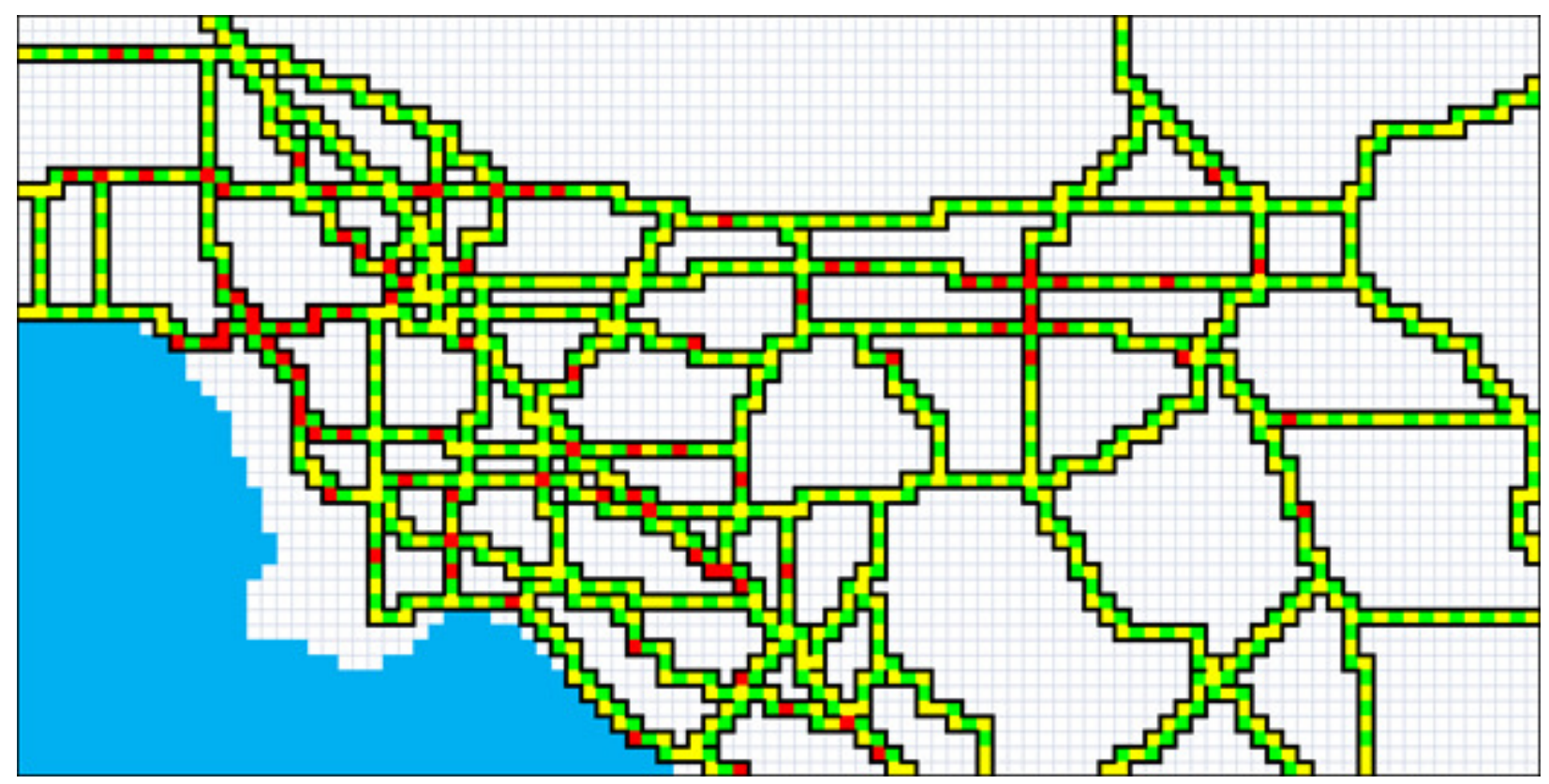

Figure 5: Current Topology, Including Roadway Nodes and Candidate HDF Sites

\subsection{HFS Investors}

Investors building HFSs do not explicitly take the presence of HDFs into account in the way that they do other HFSs, because the sales by an individual HDF are small ( $\sim-10$ percent) compared with those of an HFS, and its local effect on a nearby HFS is correspondingly small. In the aggregate, though, their existence does affect the investor agents' decisions. Their investment algorithms evaluate the sales at existing investor-owned HFSs to estimate sales potential at a new site, and these sales are reduced to the extent that driver agents buy from HDFs. Another factor in the decision algorithm is the growth rate of average sales per site, used to project the future earnings of a candidate HFS. HDFs influence this parameter depending upon how rapidly they are introduced. As a result of these factors, investors may build fewer HFSs and/or delay the building of HFSs. On the other hand, the presence of HDFs may spur more driver agents to buy hydrogen vehicles, thus increasing demand for hydrogen and encouraging the HFS investors to build more stations, in a positive feedback ("virtuous") cycle. 


\subsection{Driver Agents}

Four major changes in driver agent characteristics were necessary to have them respond appropriately to the new mixture of both HFSs and HDFs. First, HDFs are expected to be "barebones" facilities without amenities such as convenience stores and car washes and will not appeal to everyone. Consequently, before the simulation is run, driver agents are randomly binned into one of three categories: (1) those who will not go to an HDF even if the hydrogen selling price is less than that of HFSs, (2) those who are concerned predominantly with price and will buy fuel from whatever facility costs them the least, and (3) a middle group who will patronize HDFs, but only if they save a sufficient amount to make up for the lack of amenities.

The second change accommodates a key difference between HFSs and HDFs. HFS owners, to avoid inconveniencing and likely alienating their customers by not having fuel available at all times, will resort when necessary to trucking in extra hydrogen, which they sell at a reduced profit or even a loss. HDF operators, whose business case considers the production of hydrogen as only one of three activities (i.e., hydrogen, heat, and power), may not feel this obligation. Consequently, driver agents, when planning their trips, cannot count on any given HDF having fuel when they stop there. Thus, if a driver agent must purchase hydrogen in order to make a particular trip and the only appropriately located stations are HDFs, he will not make the trip in a hydrogen vehicle (and will incur inconvenience as a result). However once on the trip, if he can save money by refueling at a HDF - without running the risk of being stranded if it turns out to not have fuel - he will do so if he is of an agent type that is willing to patronize HDFs.

The third change results from another characteristic that distinguishes HDFs from HFSs, namely that they may not be located at spots most convenient for motorists. Driver agents have been given the ability to detour from their planned route to refuel at HDFs. This behavior is described in more detail later in this section.

The fourth change is a consequence of the expected low capacity of HDFs and the fact that any given HDF can run out of fuel. As discussed earlier in connection with the "millicar" and "multiple personality" concepts, modeling a diverse scenario with each driver agent representing many $(1,000)$ real drivers can pose difficulties. A new problem of this type appears with the introduction of HDFs. To illustrate this problem, assume that an HDF has a hydrogen production capacity of $73 \mathrm{~kg} /$ day, or $6,660 \mathrm{~kg} /$ quarter. Because a single driver agent represents some 1,000 real drivers, he must buy enough fuel to account for the individual purchases of those 1,000 drivers. An agent who owns 100 percent HPVs and is almost at the worst-case "desperate to buy" level (10 percent fuel remaining) upon reaching an HDF will require close to $1,000 * 0.9 *$ $6=5,400 \mathrm{~kg}$ of hydrogen. To make matters worse, to reduce computation time, the normal procedure in the past was to bundle together a suite of 39 trips (19 commuter trips and 20 "random" trips) into a "megatrip" and then simply multiply the results of this single megatrip by a factor greater than 1 chosen to make the global quarterly mileage average for all driver agents of a given income level equal to the specified average. For upper-income agents (who drive more than other agents), the factor is about 3.5, so such an agent's purchase could be as much as $3.5 *$ $5,400=18,900 \mathrm{~kg}$, or 2.8 times the entire quarterly production of the HDF! 
Of course, this is a worst case, one unlikely to occur in our simulations. Even an upper-income agent who purchased nothing but HPVs every year would not have turned over his entire fleet until 9 years had passed, using the standard input parameters. Nevertheless, owning even a significant fraction of HPVs could cause a driver agent to purchase a large fraction of an HDF's output, leaving little or no fuel for other agents for the rest of the quarter, or even for himself on a later trip.

Mitigating this problem by reducing the maximum possible purchase to, say, 10 percent of the HDF's production would require either increasing the number of agents from 1,000 to 1,000* $2.8 / 0.1=28,000$ or increasing the number of trips per megatrip from 39 to $39 * 2.8 / 0.1=$ 1,090. Either change would greatly increase the run time of the simulation. To get around this problem, we use an optional dynamic megatrip assignment procedure. Before each driver agent begins his series of trips for the current time step, the program calculates the current fraction of HPVs in his millicar fleet and divides that number by a threshold input parameter. For example, if the fraction is 0.25 and the input threshold parameter is 0.1 , the result is 2.5 . The result is rounded to the next higher integer (3) and is used to multiply the base number of trips (normally 19 , with resultant value of 57). In this way, the simulation can proceed quickly in the early years and for driver agents with low HPV fractions in general, yet still prevent any single purchase from exceeding a desired fraction of HPV quarterly output.

With these four changes, a summary of a driver agent's trips is as follows. Each quarter, a driver agent's current HPV fraction is calculated and used to determine the number of trips in his "megatrip." The trips are chosen from a portfolio of 20 "random" destinations and 1 assigned job location destination. (The "random" destinations are chosen from cells according to their population density, as well as from a number of "attractors" such as airports and shopping centers.) The resulting round trips to each destination are strung together to form a megatrip of the required number of trips and the specified random-to-commuter trip ratio.

Upon starting each trip in the megatrip, the driver agent instructs his GPS system to calculate his route and identify the optimum place(s) along the route to refuel if necessary, based on his expected fuel level and considering only HFSs. These locations are chosen to strike an optimal balance between Worry (fear of running short of fuel) and Inconvenience (stopping earlier than necessary to refuel). If necessary, the agent will make a special trip to the HFS closest to his home to refuel before commencing the journey. If the HFS lies outside his neighborhood, defined as a radius of 3 miles around his home, such a trip will cost the driver both money (in per-mile operating costs) and Inconvenience. Of course in many cases, for short trips the agent will not need to refuel. On the other hand, if he needs to refuel in order to make the trip and it is impossible to do so before reaching his "desperate-to-buy" fuel level even with a special refueling trip, the agent will not make the trip by HPV, and he will incur inconvenience as a result. Similarly, he will not make the trip if he cannot return home again with at least enough fuel to reach the nearest HFS.

The agent now starts on his trip, assured that he will be able to find any needed hydrogen along the way. However, if HDFs exist along his route or close to it, he will stop at them or even detour out of his way in preference to his planned HFS stops if by doing so his "fully accounted" cost is less. The full accounting includes the following: 
- Extra fuel and operating cost and inconvenience incurred in making a detour.

- Savings in fuel cost, referenced to the amount that he would spend if he purchased fuel at the closest HFS (can be negative).

- Reduction in worry or inconvenience.

- Perceived value of amenities foregone (e.g., convenience store). This is zero for some drivers, "infinite" for a second group who will not patronize HDFs, and a specified value for the third group.

Even if the agent does not need to refuel on his trip, he will stop at (or detour to) an HDF if either (1) the above fully accounted HDF fuel cost (including "early buy" inconvenience) is less than the cost of purchasing the same amount of fuel from an HFS; or (2) the driver is already beginning to worry and the fully accounted cost is less than or even up to a slight amount (\$3) more than same amount of fuel from an HFS (because worry will only increase if he does not buy immediately).

It is perhaps useful at this point to recognize again that these involved calculations are something that very few human drivers would actually perform. Nevertheless, they are intended to mimic in the computer world - the conscious or unconscious qualitative calculations that any driver must perform to some degree in reaching a decision as to whether to stop.

Throughout his trips, the driver agent keeps a tally of his worry (from having to buy with a fuel level substantially below his comfort zone) and his inconvenience stemming from both having to buy before he is ready to and buying from an HFS that is selling hydrogen beyond its production capacity (using trucked-in hydrogen), which could result in refueling delays. He also tallies cost savings resulting from purchases at HDFs rather than HFSs. All these factors will figure into his decision (or rather his decisions, since he has multiple personalities) for or against HPVs when the time comes each year to purchase more millicars. 
Analysis of CHHP as a Bridge to a Hydrogen Transition

This page intentionally blank 


\section{Results}

\subsection{Base Case Configuration}

Our model has a large number of parameters that may be tuned to examine their effects on the outcome of the transition to hydrogen. Before using the model to investigate the role that HDFs might play in the transition, we first establish a reasonable configuration for a case without HDFs; this case will serve as a basis for comparison. It is important to recognize that the benchmark case is not a prediction of the most likely outcome and also that it is not entirely databased. Most tangible benchmark parameter values (such as the capital cost of a hydrogen station or the variable cost to produce $1 \mathrm{~kg}$ of hydrogen) originate from DOE's H2A Project [16,17]. Table 1 provides a summary of the values used for such parameters.

Table 1: Selected Base Case Parameter Values

\begin{tabular}{llll}
\hline \multicolumn{1}{c}{ Parameter } & \multicolumn{1}{c}{ Value } & Units & \multicolumn{1}{c}{ Source } \\
\hline HFS Capital Cost & & & \\
HFS Fixed O\&M Costs & $4,375,383$ & $\$$ & {$[16] /$ Refueling Station, B162 } \\
HFS Salvage Value & 215,383 & $\$ / \mathrm{yr}$ & {$[16] /$ Refueling Station, B184 } \\
HFS Variable Production Cost & 0 & $\$$ & {$[17]$} \\
HFS Selling Price & 1.308 & $\$ / \mathrm{kg}$ & {$[16] /$ Results } \\
HFS Price to Truck-in Hydrogen & 5.47 & $\$ / \mathrm{kg}$ & {$[16] /$ Results, E17 } \\
HFS Maximum Capacity & 1500 & $\$ / \mathrm{kg}$ & Internal \\
HFS Effective Capacity & 1278 & $\mathrm{~kg} / \mathrm{day}$ & {$[16] /$ Input Sheet Template /C22 } \\
After-tax Discount Rate & 10 & $\mathrm{~kg} / \mathrm{day}$ & {$[16] /$ Input Sheet Template /C21 } \\
Tax Rate & 38.9 & $\%$ & {$[17]$} \\
Investment Time Horizon & 10 & $\%$ & {$[17]$} \\
Capital Equity Financing & 100 & $\mathrm{yr}$ & Internal \\
Investment Capital Funding & Unlimited & $\$$ & {$[17]$} \\
Neighborhood Radius & 5 & $\mathrm{mi}$ & Internal \\
HDF Capacity & 73 & $\mathrm{~kg} / \mathrm{day}$ & {$[5]$} \\
HDF Selling Price & 8.57 & $\$ / \mathrm{kg}$ & {$[5]$} \\
\hline
\end{tabular}

Addressing intangible parameters, such as the value a driver places on the convenience of not needing to detour from the desired route to buy fuel or the premium a driver places on owning a "green" vehicle, presents more of a challenge. To the extent possible, these values were calibrated to the results of consumer surveys conducted by Synovate Motoresearch. The remaining values were chosen first as reasonable estimates and then adjusted so that the overall progression of the simulation approximated a successful transition scenario described by the NRC [18]. A validation of the model's basic behavior is discussed in the RCF and Argonne report [1]. 
Each driver agent is randomly assigned several attributes. One such attribute is the quarter of the year during which the vehicle purchase decision will be made, with an equal number of driver agents assigned to each quarter. Another attribute is the driver agent's level of aversion to running out of fuel, which determines the level of fuel tank depletion at which the driver most wishes to fill up. Again, these values are equally distributed among driver agents. The amenity preference discussed in Section 4.3 is another randomly assigned attribute, with 20 percent of driver agents unconcerned with the lack of station amenities, 20 percent unwilling to use HDFs, and 60 percent weighting an inconvenience penalty in the decision of whether to use an HDF. Driver agent personalities, as discussed in Section 2.2.1, are randomly assigned on an agent-byagent basis, but the distribution of personality types approximates the findings of Synovate and other surveys in the literature [19]. We assign values such that the common "Go with the Crowd" mentality carries a lower weight in the decision-making process relative to the stronger "Innovator" or "Luddite" mentalities. In tests, we have found that, while using different random assignments for these attributes does vary the progression of each simulation, the ultimate outcomes do not significantly deviate from one another.

The base case utilizes a single investor. As described in the Mahalik et al. paper [4], the use of multiple competing investors can introduce additional randomness and some inefficiencies in investor siting of HFSs, and that conclusion still holds for the current version of the model. As such, we continue with the single investor agent in an attempt to isolate as much as possible the effects of introducing HDFs and reduce the need to question simulation outcomes as the effect of random events.

Because some HFSs and some HPVs are already operating today within the study area, our simulation likewise originates with some stations and vehicles in place. A group of four publicly accessible, currently operating stations serves as our "seed" stations, one each near Burbank, Diamond Bar, Irvine, and West Los Angeles; each is represented in the base case by an HFS [20]. Although the stations in operation today do not have the same capabilities as the HFS objects in our model, it is not unreasonable to expect that, as time passes and the community of early adopters supporting these stations grows (during the years occurring before the modeled start of our simulations), the capacity available at or near each location could be incrementally expanded by the addition of new equipment. In order to identify seed driver agents, first the simulation is executed for 1 year with the seed stations in place and no seed driver agents. Afterwards, we examine the agents to find those high- and medium-income agents who have the greatest desire to own HPVs. We choose a group of agents at the top of that ranking to subsequently start the simulation already owning some hydrogen millicars, such that the number of seed HPVs on the road is approximately 4,000, or 0.05 percent of the fleet. While seed driver agents start the simulation with some hydrogen millicars, each is unbiased in subsequent vehicle purchase decisions.

\subsection{Base Case Results}

Figure 6 shows the results of the base case. The adoption of HPVs starts slowly but accelerates at a smooth pace, ending with a fleet penetration level of 31 percent after 20 years. If this simulation were to continue, the curve would reach an inflection point and take on the S-curve 
shape typical of new technology adoption. The S-curve shape can already be seen in the rate at which drivers prefer HPVs, because this is a reporting of instantaneous tastes and is not subject to the many-year delay required to replace a durable technology. We see that the investor agent smoothly adds refueling capacity in a pattern similar to the penetration of vehicles into the fleet, although with a stepping effect since HFSs are added annually while driver agents purchase vehicles quarterly.

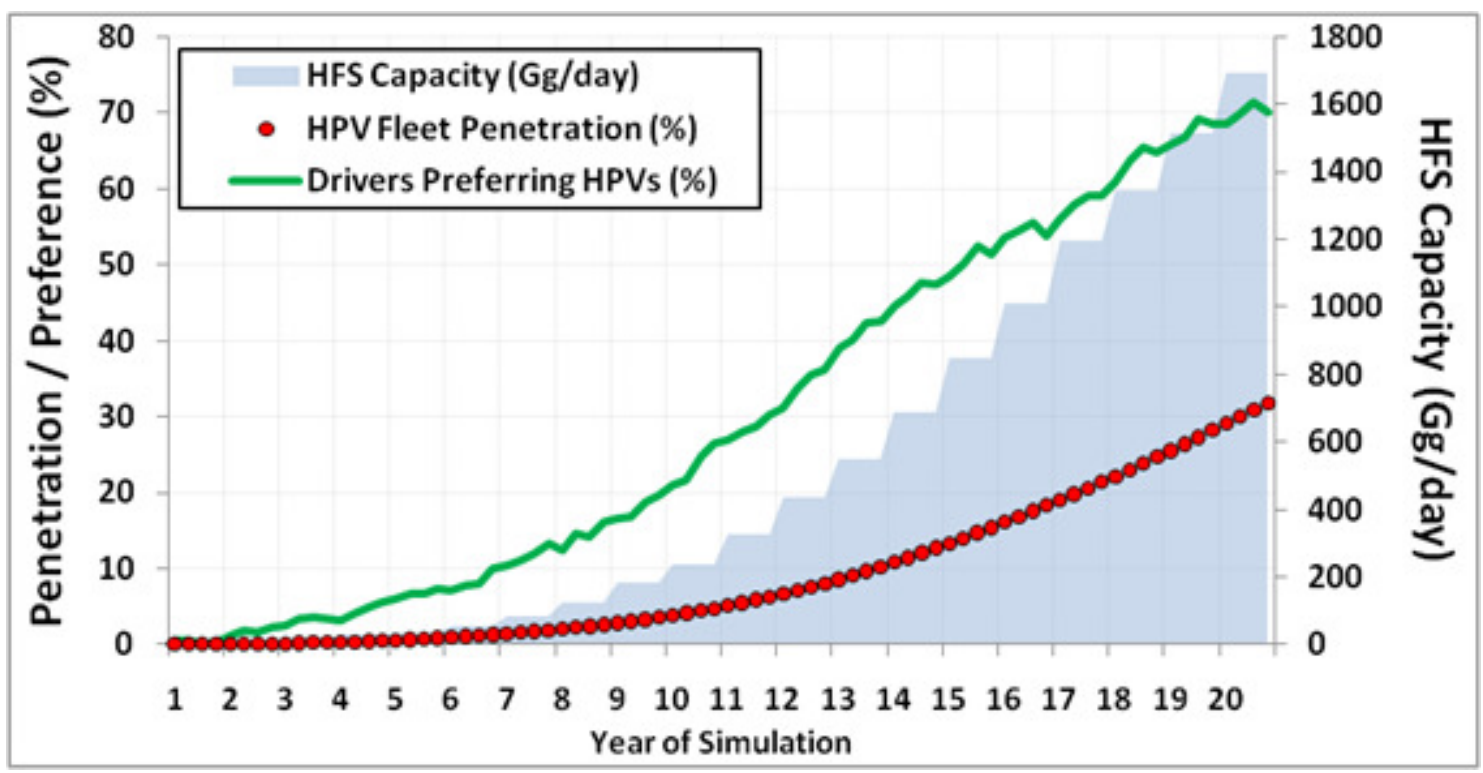

Figure 6: HPV Fleet Penetration, Drivers Preferring HPVs, and Aggregate HFS Capacity in the Base Case

Several general factors contribute to this particular progression of the simulation. Each factor makes HPVs more attractive to driver agents as time goes on and accelerates the rate of adoption. First, there is an exogenously defined decrease in the distance-independent cost disadvantage of HPVs over the first 2 years. In the first year, we assign a value of $\$ 4,000$, which drops to $\$ 2,000$ in the second year and then to $\$ 1,000$ for all subsequent years. This early drop is meant to reflect both the consumers' declining reluctance to invest in a new durable technology as they wait for the technology's viability to be proven, as well as real dollar declines in the sale prices of the new vehicles early on. The remaining standard price disadvantage corresponds to the difference in sticker price relative to the price of a comparable conventional vehicle after rebates and/or subsidies have been applied. Second, there is also an endogenous vehicle price drop that is a function of the number of HPVs sold, reflecting economies of scale in the manufacturing process. Third, as the investor agent builds more HFSs, driver agents experience a decrease in the frequency and intensity of worry and inconvenience events. Fourth, as individual HPV drivers interact with neighbors, coworkers, and society at large, acceptance of and enthusiasm for the new technology grow (this is a positive effect for the majority of our driver agents, but not all; the Innovator personality is most enthusiastic about a technology before it becomes mainstream). 
Figure 7 shows the geographic distribution of HPVs at 5, 10, 15, and 20 years. Each cell is colored according to the total number of HPVs owned by the driver agents living in each cell. Grey cells are those in which no driver agents have converted to hydrogen. HPV adoption starts strongly in the highly populated western area and spreads to the more sparsely populated eastern area over time.

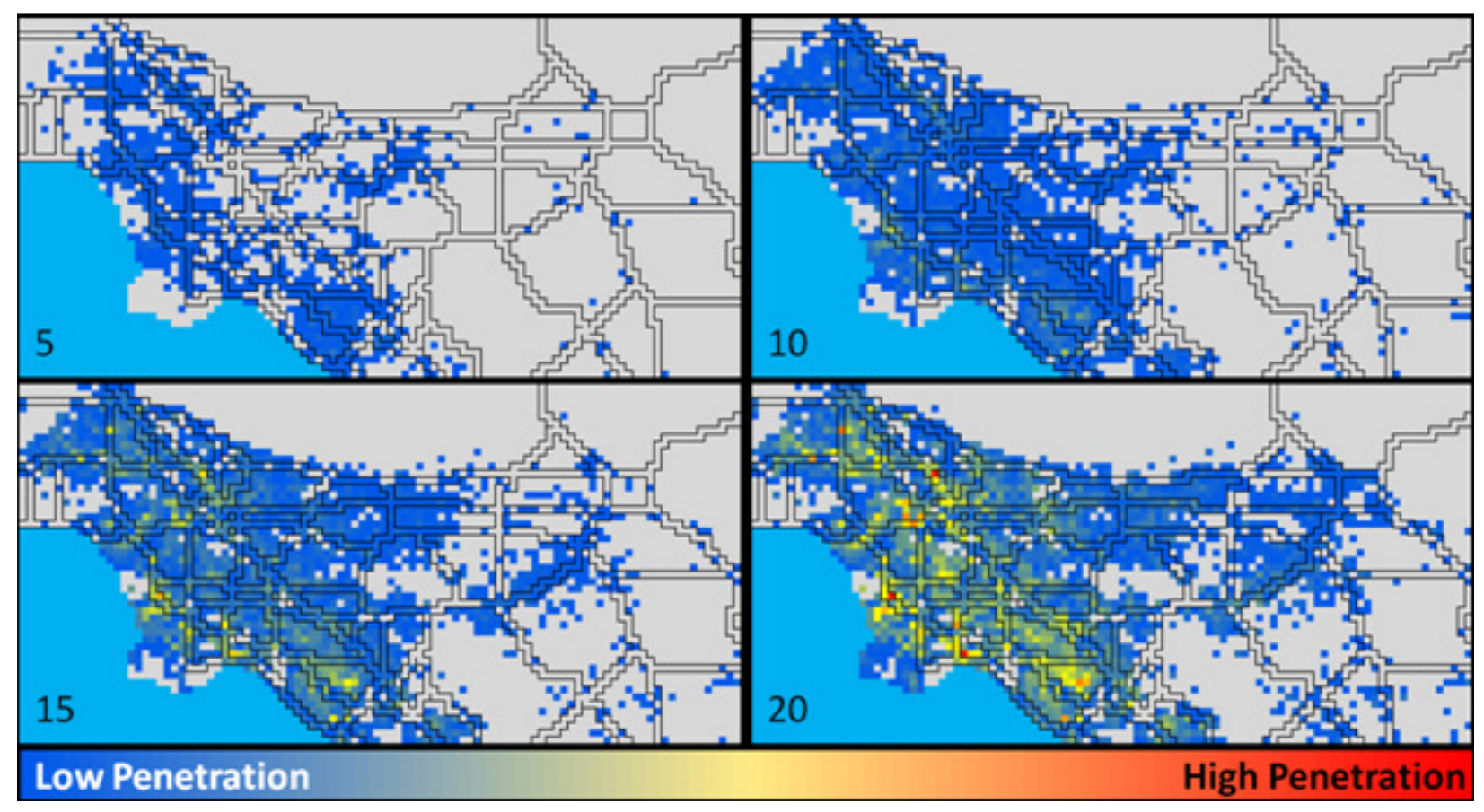

Figure 7: Geographic Distribution of HPVs in the Base Case

\subsubsection{Investor Variations}

A curious observation made in the base case is that the investor agent seems to concentrate large amounts of supply into relatively few locations. By the end of the simulation, only 80 of the 553 candidate locations have an HFS, and most of those selected locations each have more than one HFS. This macro-level result can be interpreted on the micro level as meaning that more than one station exists within the same square-mile area, or that the investment serves to expand the daily capacity of an existing station and/or build higher capacity stations. While certainly some locations will have more demand for hydrogen than others, it is also an intuitive expectation that by spreading the supply of fuel to cover a wider area, the appeal and success of HPVs should increase. To test this expectation, we conduct a run in which we take manual control of the HFS placement. We place the same number of stations as the investor agent and follow the same temporal construction schedule of those stations, but we make the supply available over many more locations. We take a simple, yet intuitive, approach to selecting the station locations: we rank the candidate locations according to the amount of traffic at each and work down the list, starting with the most heavily traveled location. The 30 percent of locations ranked lowest are 
excluded. Once we reach the bottom of the list, we start again from the top. Figures 8 and 9 show the results.
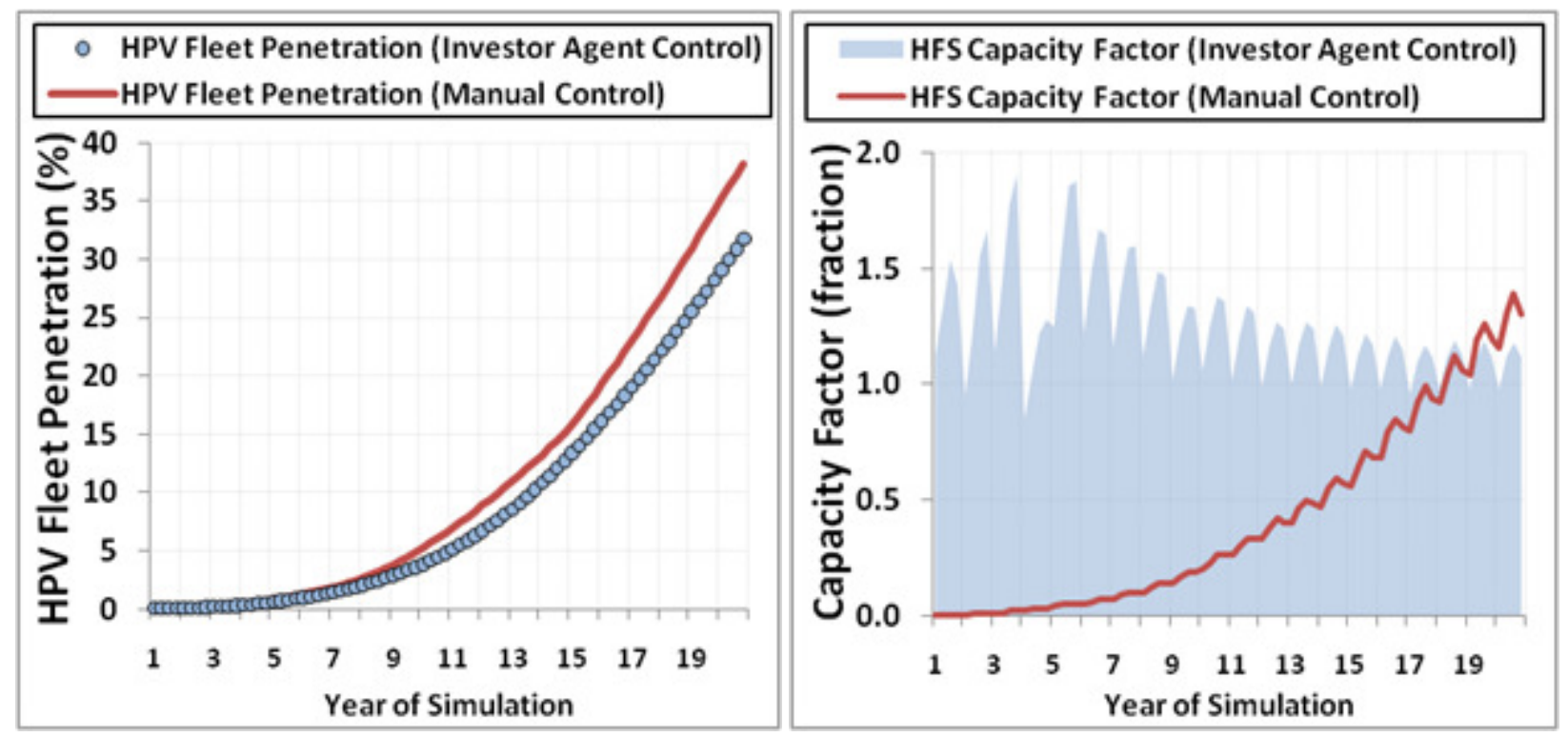

Figures 8 and 9: HPV Fleet Penetration and Average HFS Capacity Factor When HFS Rollout Is under the Control of the Investor Algorithm (8) and a Simple Heuristic (9)

Not surprisingly, driver agents are consistently happier with this arrangement, and by the end of the simulation, an additional 6 percent of the fleet has been converted. Although the most popular locations are consistently overcrowded and driver agents choosing to use those stations suffer in the form of a high inconvenience penalty, all other types of worry and inconvenience events are considerably lower for the average driver agent. However, from the perspective of the investor agent, this arrangement would be an unsustainable financial disaster. The average capacity utilization of the HFSs is very low throughout most of the simulation - far too low for most of the stations to break even, let alone turn a profit. The investor algorithm does a much better job of selecting when and where supply is needed, keeping stations well-utilized and in the threshold of profitability by neither selling too much trucked-in hydrogen nor building new stations before they would be able to survive on their own. On an individual basis in the alternative case, some stations are selling an unrealistic quantity of trucked-in supplies, while others are being virtually ignored. Such under-utilized stations are the cause of the higher fleet penetration in this case; fuel is now accessible to driver agents who otherwise would not purchase HPVs, but in these instances, full-scale HFSs are not practical. It is for such drivers that less expensive, smaller scale solutions such as HDFs hold the biggest promise. Toward the end of the simulation, the average utilization exceeds 100 percent and continues an upward trend; at this point, the average station is losing an excessive amount of revenue by selling trucked-in supplies, and more stations should be built. 
While the behavior of the investor agents may seem odd at first glance, upon further inspection, it becomes clear that the algorithm is doing just as intended: placing HFSs at just the right time and location to sustainably serve growing demand and make a profit doing so.

\subsubsection{Sensitivity to Agent Interactions}

The exhibition of reasonable behavior by our agents is by no means guaranteed for every conceivable case. By manipulating a few input parameters, it is possible to produce a case in which, for example, exactly 20 percent of driver agents choose to purchase HPVs regardless of the price and availability of hydrogen fuel, or in which an investor agent builds 45 new stations each year regardless of how much demand exists. Indeed, the nature of the model (or virtually any model for that matter) is such that we could obtain any result desired through the careful setting of input parameters. However, in our base case, neither driver nor investor agents are "locked in" to following any particular course of action. The agents in our base case each respond to the actions taken by the others in a reasonable manner, consistent with the rules of the simulation that we have described. To demonstrate, we simulate a series of hypothetical cases in which we manually take control of the infrastructure development and observe how the driver agents react.

Figure 10 shows the results in terms of fleet penetration of HPVs. As shown earlier, the base case achieves roughly 30 percent penetration by the end of the simulation. In the case One HFS per Candidate, we start the simulation with one HFS in each of the 553 candidate locations and do not allow the construction of any additional HFSs. The transition gets off to an excellent start because of the wide availability of fuel, but soon the supply becomes taxed and drivers must deal with overcrowded stations. The general appeal of HPVs reaches a peak after 3 years (not shown in the figure) and declines from there, so the rate of penetration steadily decelerates to a plateau. By the end of the simulation, this case is less successful than the base case.

In the case Seed Stations Only, the four seed HFSs are the only ones that exist throughout the simulation. While some drivers do purchase HPVs, market penetration is never able to reach beyond "niche" status. In the case Ubiquitous Hydrogen, unlimited HFS capacity is available at each candidate location from the start of the simulation. The penetration accelerates quickly as in the One HFS per Candidate case, but here driver agents do not experience the negative feedback of overtaxed supply, and so the transition is much more successful. In All Drivers Prefer Hydrogen, every driver agent is set to always opt for HPVs and purchase them as quickly as possible. This case serves mainly to illustrate the rate at which the fleet turns over. It is interesting to compare the last two cases; simply because hydrogen fuel is readily available does not mean that all of our driver agents will prefer to switch to the newer technology. For some drivers who do not travel many annual miles, the per-mile savings on fuel is not enough to make up for the higher cost of the vehicle. Other drivers might have a strong personality that either eschews or embraces the currently dominant technology. These exercises demonstrate that our driver agents are indeed quite sensitive to availability of hydrogen fuel, and so the ability to alter the progression of the transition via the introduction or withholding of refueling infrastructure does exist. 


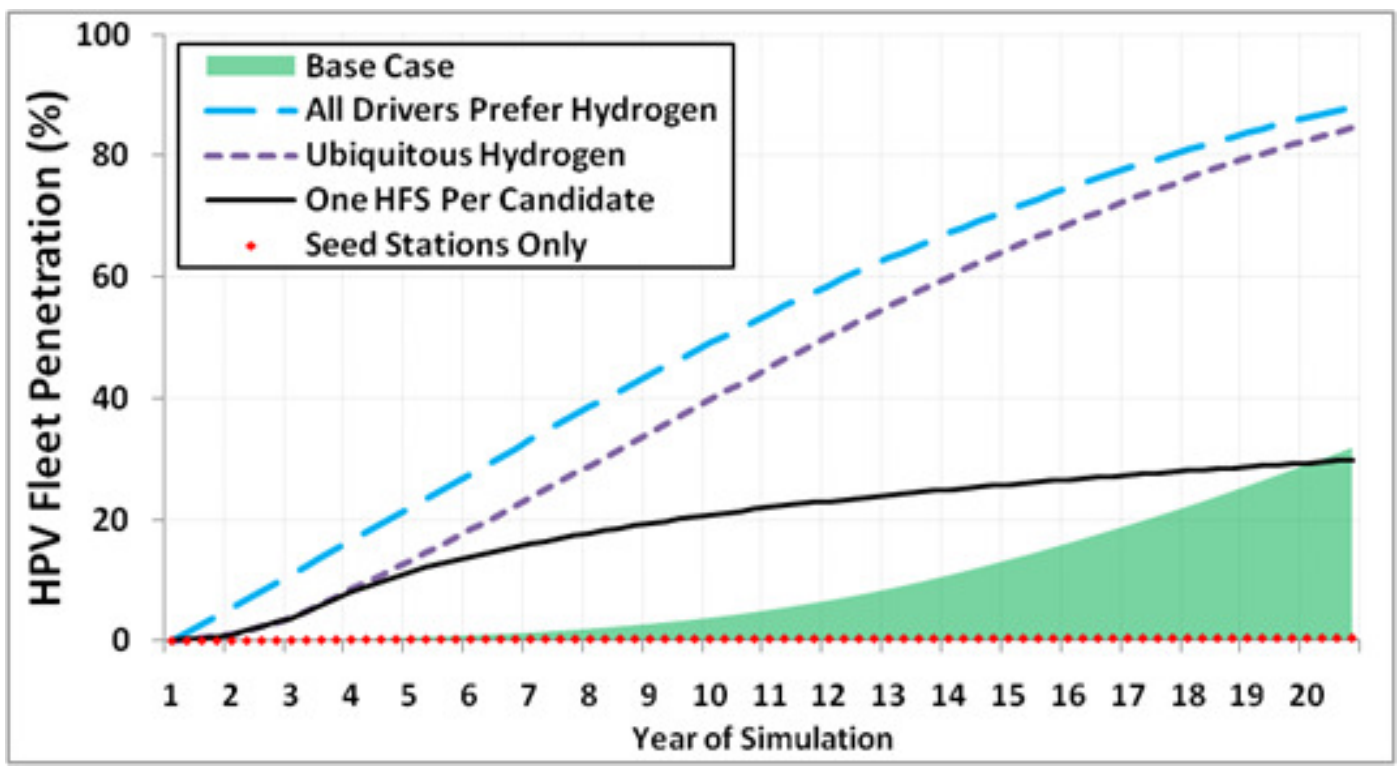

Figure 10: Fleet Penetration of HPVs under Various Hypothetical Scenarios

\subsection{Introduction of HDFs}

To investigate the role that HDFs might play in the transition, we simulated and analyzed a large number of different cases. In each scenario, we varied one or more parameters and tested a range of possible values for those parameters to determine their effect on agent behavior and the overall progression of the transition. Because the focus of this study is on the role of HDFs, we have not examined every parameter of the model. For instance, each driver agent is exogenously assigned one quarter of the year during which that agent makes a vehicle purchase decision. While varying this parameter for each driver would certainly affect to some extent the outcome of the simulation, doing so would not illuminate the potential effects of having HDFs present. By contrast, varying the fuel storage capacity of HPVs (and thus their effective range) would likely make the geographic dispersion of refueling sites more or less important to the success of the transition, and so the availability of HDFs likewise becomes more or less important.

It is not feasible nor would it be compelling to detail every variation simulated during the study. Instead, we present a collection of important trends and findings that we observed to be consistent over many variations and highlight specific cases to illustrate each.

The default parameters characterizing HDFs are based on the report and accompanying spreadsheets produced by NREL to investigate the economic basis for CHHPs [5]. The report details a case study of a $200-\mathrm{kW}$ molten carbonate fuel cell that is used to produce $29,700 \mathrm{~kg} / \mathrm{yr}$ (81 kg/day) of hydrogen, at a cost of $\$ 8.57 / \mathrm{kg}$ as dispensed [5]. We choose HDFs of the same capacity for the first example and assume that 10 percent of the hydrogen produced will be used on site, leaving $73 \mathrm{~kg} /$ day for sale to the public.

To find the upper effects that such HDFs could have on the transition, we simulate a hypothetical case in which all candidate HDFs are in place from the start of the simulation. We find that our 
agents choose not to use the HDFs, and the transition is unaffected. The selling price relative to the $\$ 3.30 / \mathrm{kg}$ charged for fuel from an HFS is too expensive. Driver agents living far from HFSs, for whom the HDFs would be their primary source of fuel, choose not to purchase hydrogen vehicles because of the expected expense. Drivers who live near HFSs choose to accept the inconvenience of going out of the way to refuel rather than pay the higher HDF price. In the following exercises, we identify the circumstances necessary for driver acceptance of HDFs.

\subsubsection{HDF Fuel Price}

In terms of HDF availability, no rollout scenario can rate higher than the hypothetical case just described (all candidate sites operate an HDF from the start of the simulation). In the subsequent cases, we examine more realistic rollout scenarios in which not every candidate site makes an HDF available, and in which the number of HDFs grows over time. The number of HDFs built, the timeframe in which they become operational, and the locations at which they are sited are all important factors that affect their overall impact on the outcome of the transition. While specific examples cited throughout this section may be the product of only one particular HDF rollout scenario, the general conclusions drawn have been found to be true (to varying extents) over a variety of rollouts.

Not surprisingly, the price of fuel is a critical factor in consumer acceptance of HDFs. We find that the transition represented in our base case can indeed benefit from the introduction of HDFs, as long as the price of fuel is acceptable to consumers. The price of HDF fuel must be below $\$ 5 / \mathrm{kg}$ before our drivers begin to rely on HDFs for anything other than "emergency" fuel purchases (i.e., when patronizing an HDF can allow the driver agent to either avoid the greater inconvenience of having to make a special trip to buy fuel from an HFS or otherwise forgo taking a particular trip because of the lack of convenient HFSs). For any given rollout scenario, lowering the fuel price further entices more and more driver agents to view HDFs as a viable method for obtaining fuel. Those agents may then choose to purchase HPVs when they otherwise would not have, with the end result being that HPVs attain some higher degree of fleet penetration. Depending on the HDF price, the ultimate acceleration of the base case transition is positive but not dramatic, ending with anywhere from 1 to 8 percent additional fleet penetration. Lower prices (down to $\$ 1.00 / \mathrm{kg}$ ) naturally net the highest gains, but the incremental return from each price drop is lower and lower; at prices below those of HFSs, the HDFs are so popular that they increasingly sell out of fuel, so the benefit of their presence to the consumer is likewise diminished.

Although there is significant potential for improvement in our base case transition (as demonstrated earlier), it is also is fairly successful in its own right - even without the benefit of having HDFs present. Obviously, every driver agent who prefers HPVs, even in the absence of HDFs, is one fewer agent for whom the presence of HDFs might act as the deciding factor when purchasing a vehicle. In general, we observe that the less successful the transition is in the absence of HDFs, the greater is the potential for the transition to accelerate via their introduction. To demonstrate, we start by making a simple modification to the base case assumptions: increase the fixed price of HPVs such that their fleet penetration drops to about 10 percent when HDFs are not present. Under these new circumstances, HDFs have a greater potential to positively 
affect the transition, increasing the fleet penetration by up to 16 percent in some cases. However, even under this alternative scenario, drivers choose not to use HDFs if they sell at the price of $\$ 8.57 / \mathrm{kg}$. Figure 11 summarizes one such set of results.

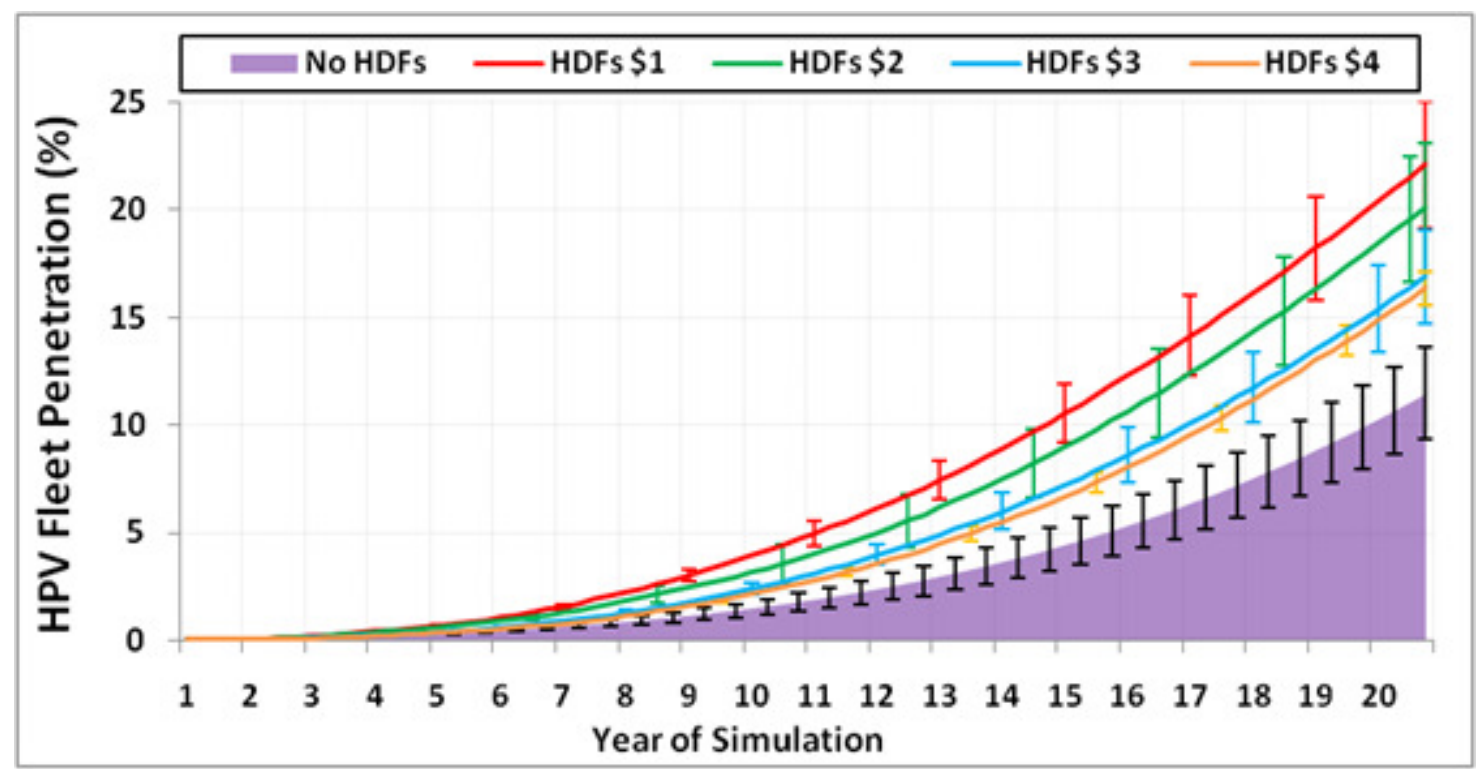

Figure 11: HPV Fleet Penetration under the Base Case and with HDFs Selling Hydrogen at Various Prices

It must be emphasized that that the difference in the end state of any of these scenarios is not wholly attributable to the difference in fuel price. Because our simulation is a complex adaptive system, many factors contribute to the final outcome. Perhaps the most significant of these is the course of action taken by the HFS investor agent. Differences in the price of HDF fuel can cause different driver agents to embrace or reject HPVs. Even small variations in which individual driver agents adopt HPVs can lead the investor agent to build an HFS in one given location over another, or in both locations, or in neither location. Once the first deviation in HFS siting occurs, more deviations naturally follow as driver agents react to the presence or absence of new HFSs, investor agents react to the demand generated by those driver agents, and so on. Additionally, random numbers are used to choose driver agents' trip sequences and certain other factors. The curves shown are the average of four runs, each using a different "seed" that determines the sequence of random numbers generated. (Repeating a run using the same seed always produces exactly the same output, of course.) The error bars shown on each series represent the standard deviations from the mean of the sample. ${ }^{5}$ The growth in the error bars as penetration increases illustrates the tendency of a small difference in the early stages to increase - both absolutely and fractionally - as a result of the positive feedback between driver agent HPV purchases and investor agent siting of HFSs. Thus, in the early years, the differences between the curves are

5 S.D. $=\sqrt{\frac{\sum_{N}(y-\bar{y})^{2}}{N-1}}$ 
statistically significant, but after a number of years the random error grows to where this is no longer the case. This is a general tendency of any simulation that is subject to random fluctuations and positive feedback.

\subsection{2 "Intelligent" Placement of Fewer HDFs}

Clearly, the more HDFs brought on-line early in the transition period, the happier driver agents will be, because they will be able to refuel more conveniently, and if HDF prices are lower than HFS prices, they will save money as well. While a surfeit of HDFs may be desirable from the driver agents' point of view, an HDF that sells little or no hydrogen can hardly be justified economically. Because (in contrast to HFSs) HDFs are located and brought on-line exogenously, an "intelligent" approach to siting such stations is desirable. As an example of how this might be done, we ran a simulation in which all 121 HDFs were brought on-line in the first year, selling hydrogen at the same price $(\$ 3.30 / \mathrm{kg})$ as HFSs and with the default daily production capacity of $73 \mathrm{~kg}$ /day of hydrogen. As shown in Figure 12, sales vary widely among the HDFs: some begin to sell out in the first year of the simulation; others never sell any hydrogen at all. Over time, as more drivers purchase HPVs, there is a general tendency for HDFs to sell more hydrogen, but in many cases sales fluctuate, likely as a result of a new HFS being located nearby. Sales figures for all HDFs were collected and analyzed to determine in what year their sales first exceeded 20, 40, or $60 \mathrm{~kg}$ /day. ${ }^{6}$ In subsequent runs, HDFs were brought on-line in the years in which they met the various benchmarks (referred to hereafter as Protocols 20,40, and 60). While such an experiment cannot be done in the real world, it nevertheless seems a plausible representation of the results that might be obtained from detailed forecasting. The results are shown in Figure 13. Note that at the end of 20 years, the three protocols placed 102, 94, and 81 HDFs, respectively. While bringing all HDFs on-line in the first year results in the highest penetration rate, adding HDFs more slowly and judiciously does almost as well.

${ }^{6}$ In cases where $N \geq 2 \mathrm{HDF}$ are associated with a single node, the threshold for the first HDF is $20 / N \mathrm{~kg} / \mathrm{day}$; for the second it is $20 /(N-1) \mathrm{kg} / \mathrm{day}$, and so on. (A similar procedure is used for the $40-$ and $60-\mathrm{kg} / \mathrm{day}$ benchmarks.) 


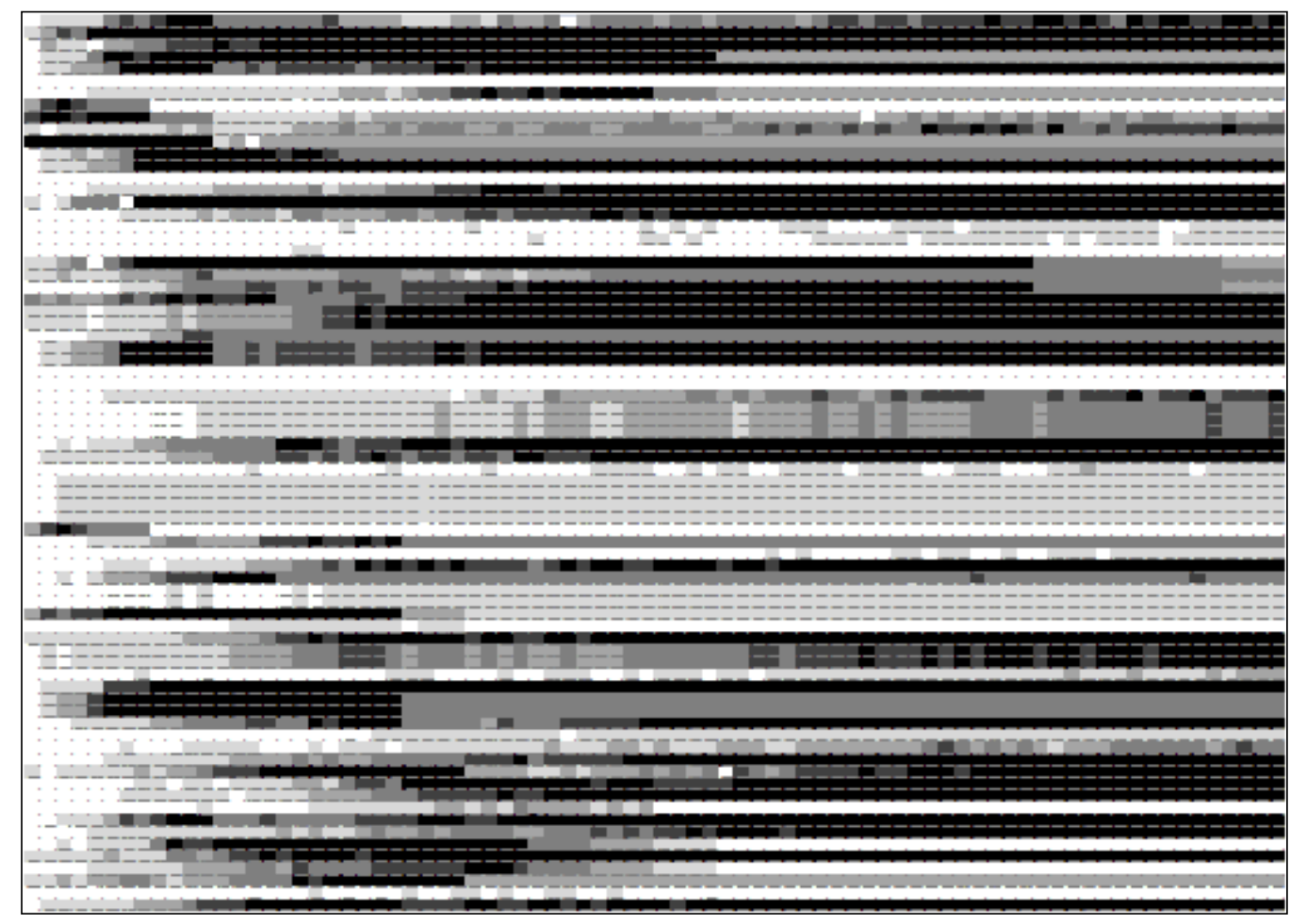

Figure 12: Sales for 74 HDFs Randomly Picked from the Total Population of 121 (Each HDF's sales are listed on a separate row, with each of the 80 1-quarter time steps of the simulation shown as a separate column running left to right.

White cells indicate zero sales; black cells represent demand equal to or exceeding the $73 \mathrm{~kg} /$ day of the HDFs. Different shades of grey represent sales in the ranges $0-20,20-40,40-60$, and $60-73 \mathrm{~kg} /$ day.)

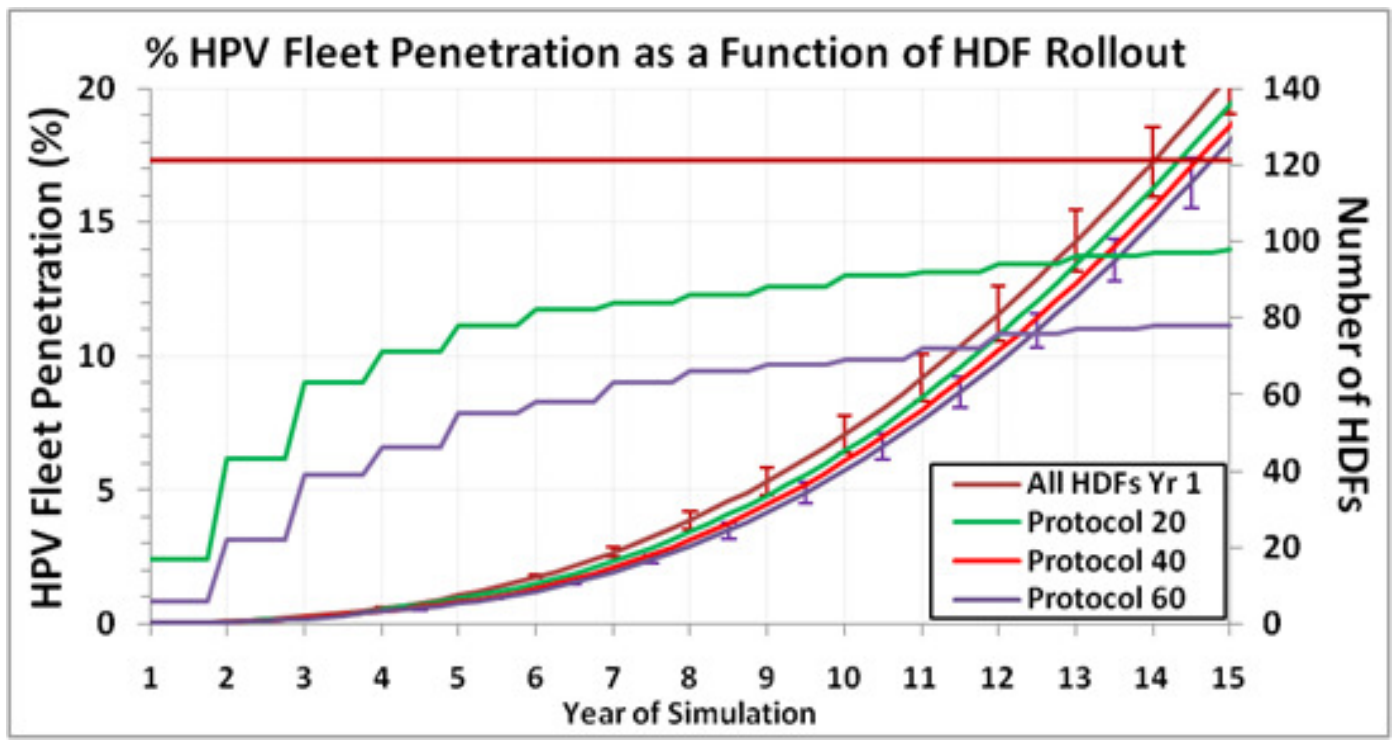

Figure 13: Penetration of HPVs into the Fleet for (a) All 121 HDFs Brought On-line in the First Year (brown curves), and (b) Smaller Numbers of HDFs Brought On-line in

Accordance with the Protocols (The stepped curves are the number of HDFs corresponding to each protocol [right scale].) 


\subsubsection{Increased Capacity of HDFs}

Even with all HDFs brought on-line in the first year, many of them sell out of hydrogen. Because driver agents do not count on HDFs having fuel, this does not cause a major inconvenience to them. However, it does discourage their purchasing fuel from HDFs to the extent that they would like to patronize the HDFs but find they cannot. ${ }^{7}$ Placing more HDFs is not necessarily the answer to this problem, as many of the original HDFs operate significantly below capacity. In this example, the capacity of all HDFs is increased from 73 to $150 \mathrm{~kg} / \mathrm{day}$. (Obviously, one would want to increase the capacity only of HDFs that were already selling out; however, in the current simulation all HDFs have the same capacity, and increasing the capacity of belowcapacity HDFs does not affect the outcome of the simulation.) Figures 14 and 15 compare the results. By roughly doubling the capacity of the HDFs, the number of run-outs is cut significantly, especially in the middle years of the transition. The penetration of HPVs into the fleet is encouraged (although the effect is less pronounced in the early years [Figure 15]), thus increasing the rate of HPV penetration such that a slow rollout of $81 \mathrm{HDFs}$ is equivalent to the year-1 deployment of 121 HDFs.

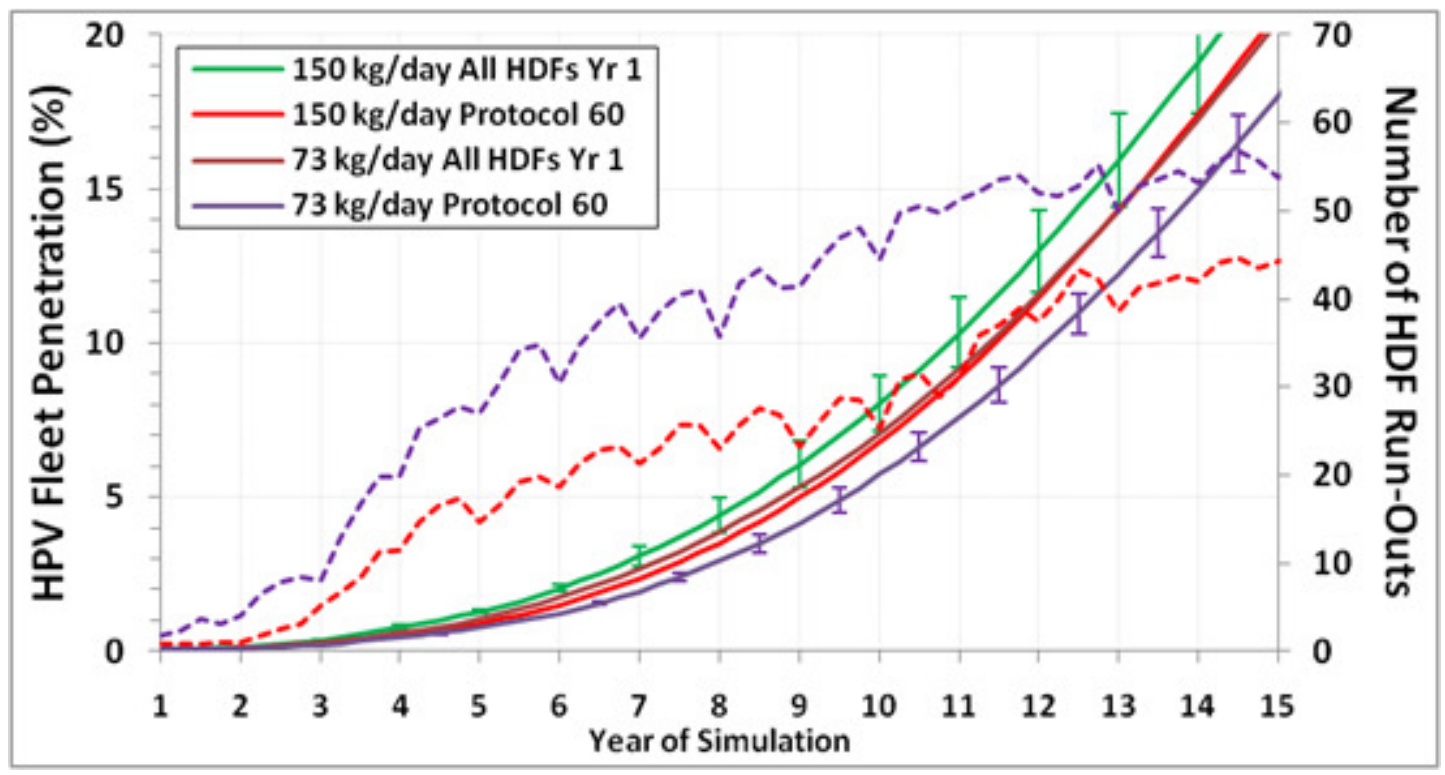

Figure 14: Effect of Increasing the Capacity of HDFs from 73 to $150 \mathrm{~kg} /$ day (The jagged curves are the number of "run-outs" each quarter. Because the model runs on a 1quarter time step, the absolute number is not meaningful.)

7 It is expected that HDFs will have a real-time internet connection and will signal when they do not have sufficient fuel on hand. The only time an HDF's running out of fuel would be a real inconvenience to a driver agent would be when he starts a detour expecting the station to have fuel, and the fuel runs out before he gets there. A quick calculation shows this to be a rare occurrence, and it is easily mitigated. Recall that even if an HDF does not have fuel, the driver agent is assured of having enough fuel on-board to reach his originally planned HFS. 


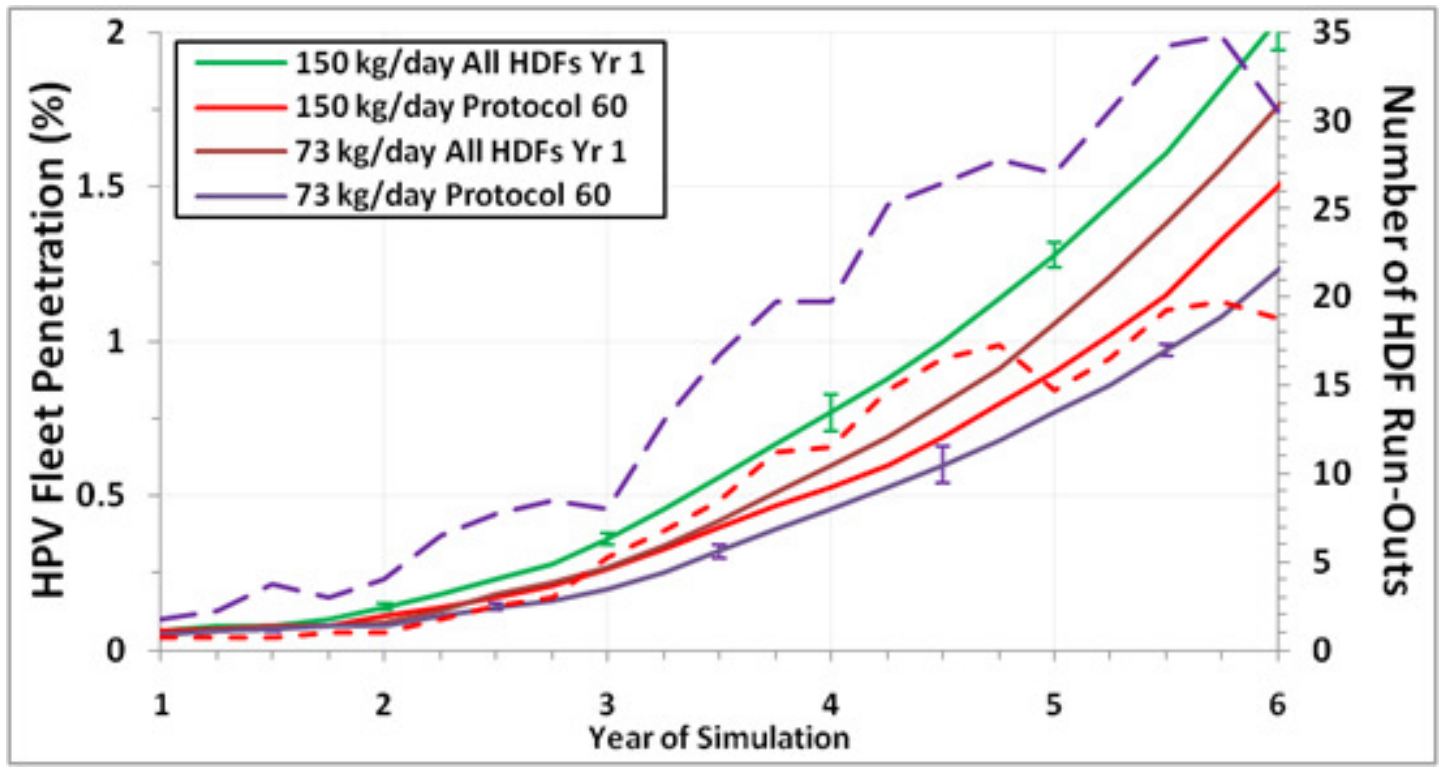

Figure 15: Effect of Increasing the Capacity of HDFs from 73 to $150 \mathrm{~kg} / \mathrm{day}$ with Expanded Scales for Early Years (The jagged curves are the number of "run-outs" each quarter. Because the model runs on a 1-quarter time step, the absolute number is not meaningful.)

\subsubsection{Early Shutdown of HDFs}

While previous examples have kept HDFs open from the time they are brought on-line until the end of the simulation, this is not necessary for a successful transition to hydrogen. The HDFs serve their purpose in the first few years of the transition and then are no longer needed, because sufficient HFSs have been built to take their place. This is illustrated in Figure 16, where HDFs are rolled out according to Protocol 20, but only through years 2 or 5 . At the end of that year, all HDFs are removed, and no new ones are added. Removing HDFs at the end of year 2 is too early, and the transition suffers; however, removing them at the end of year 5 causes virtually no slowdown in the penetration rate. 


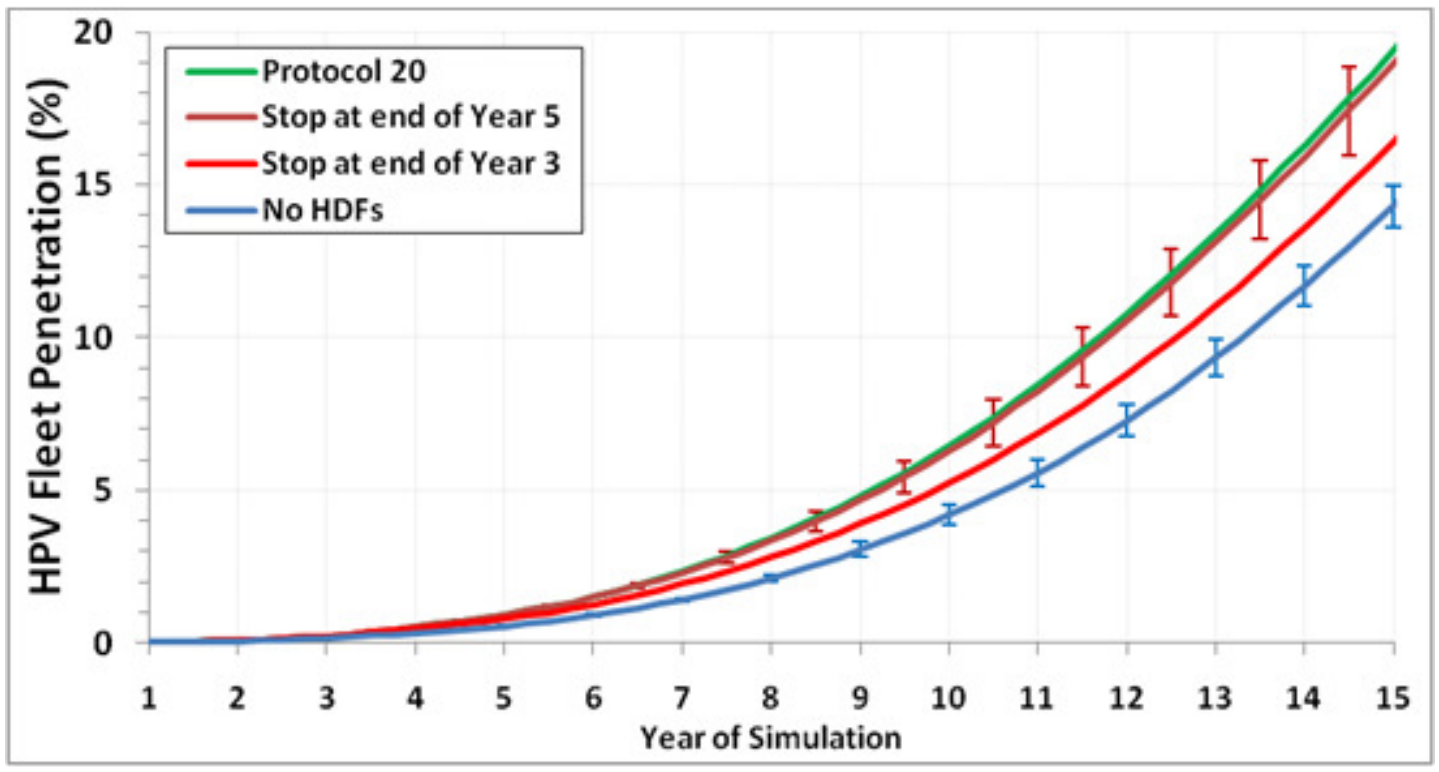

Figure 16: Effect on HPV Penetration of Removing All HDFs at the End of Years 3 and 5

\subsubsection{Effect of Drivers' Weighting of Worry and Inconvenience}

As explained earlier, each driver agent's vehicle purchase decision is based on several factors. Some of these are related to the availability of HFSs, while others (such as the purchase price of the vehicle) are not. The factors that have the most direct connection to the availability of fuel are the worry and inconvenience events. In our base case, the events that have the greatest impact on the average driver agent's decision are having to make a special trip solely for the purpose of purchasing fuel and not being able to make a desired trip by HPV. Early in the simulation when the refueling infrastructure is sparse, these events frequently occur and thus weigh heavily on the average driver agent's decision. As the refueling infrastructure grows, the frequency of these events quickly drops off. The agent also weighs the events of having to purchase fuel earlier or later than desired. These events are likewise more frequent in the beginning of the simulation, but as the refueling infrastructure grows, their frequency does not drop as sharply. Even when fuel stations are abundant, it is not always possible to refuel at exactly the desired time. (These events are less troublesome to the driver the closer he refuels to the desired time.) The cost of each event is assigned exogenously, with the "special trip" and "no drive" events typically having the highest single-incident costs.

Another type of inconvenience is experienced when refueling at a crowded HFS (one that is selling more than its production capacity via trucked-in supplies). For the average driver, this event is of least concern during the earliest stages of the transition, but as time goes on, it increases somewhat and then stabilizes. Recall that the investor agent finds success in keeping the refueling infrastructure operating near or even slightly above capacity, so this event is experienced consistently throughout most of a typical simulation. 
By running many variations on the importance drivers place on experiencing these events, we find, in general, that the more weight these events carry in the driver agent's vehicle purchase decision, the greater impact of HDFs on the success of the transition. Naturally, the base case transition suffers when driver agents are more averse to worry and inconvenience events. However, while the absolute rate of HPV fleet penetration in such situations is considerably lower, the introduction of HDFs selling at a competitive price can lead to as much as a doubling of the penetration rate after 20 years in some cases. When these events weigh heavily on a driver agent's decision, it is all the more essential to the success of the transition that they be relieved through the introduction of more refueling infrastructure, including HDFs. Although a real driver's perceived cost of experiencing these types of events may not be readily influenced by government policy, it is useful to be aware of this relationship when researching consumer attitudes.

\subsubsection{Vehicle Range}

Our base case assumes that HPVs have a range of 300 miles (6-kg tank capacity at $20 \mathrm{~g} / \mathrm{mi})$. We find that as this range changes, so does the impact of having HDFs available. The nature of this effect and its causes are closely related to the reasons discussed above regarding worry and inconvenience events. As the range of the vehicles increases or decreases, the probability of triggering worry and inconvenience events likewise increases or decreases. For example, the driver of a longer range vehicle would less frequently experience the need to make a special trip to buy fuel; because he is covering more distance between fill-ups, he is more likely to encounter a fuel station during his primary travel, and even in the worst-case scenario (he relies solely on special trips for refueling), he would still be able to wait a longer time between the special trips. Because these events occur less frequently in this case, there is a diminished opportunity to improve the driver agent's opinion of owning an HPV through the expansion of hydrogen refueling infrastructure, including HDFs. Worry and inconvenience are factored into the vehicle choice function in terms of both frequency and intensity. In the previous section, we described varying the intensities (cost) of the events; here, we describe varying the frequency. Naturally, the effects are compounded when varying both frequency and intensity.

\subsubsection{Driver Discounting of Past Experience}

In the simulation, driver agents "remember" their past experience each year when they restock their millicar vehicle fleets. They recall all past experience (e.g., worry, inconvenience) since the beginning of the simulation, but discount it exponentially. That is, the current quarter has no discount, the previous quarter's experience is discounted by a factor $D$; the quarter before that is discounted by factor $D^{2}$; and so on. Relating this factor to real life is beyond the scope of this project; indeed, it may be impossible to do so because a real driver's "discount" depends on so many factors. Nevertheless, it is important and choosing either 1 (all past experience counts equally) or 0 (driver remembers nothing of past experience) is clearly wrong. In the results reported here, we have normally used a value of 0.75 ; thus, the previous quarter's experience counts 75 percent as much as the current quarter's, experience from a year ago counts $0.75^{4}=32$ percent as much, and so on. Figure 17 compares this situation to one in which the discount 
parameter is 0.5 (previous quarter has weight of 50 percent; a year ago has a weight of 6 percent). As the figure shows, the adoption rate of HPVs is much greater in later years as drivers "forget" their earlier, less hydrogen-favorable driving experience. Of course, changing the rate at which real drivers forget the past is, at best, difficult, but the results indicate the advantages that can accrue if drivers can be given confidence that a transition is on its way and that previous, possibly negative, experiences are gone forever.

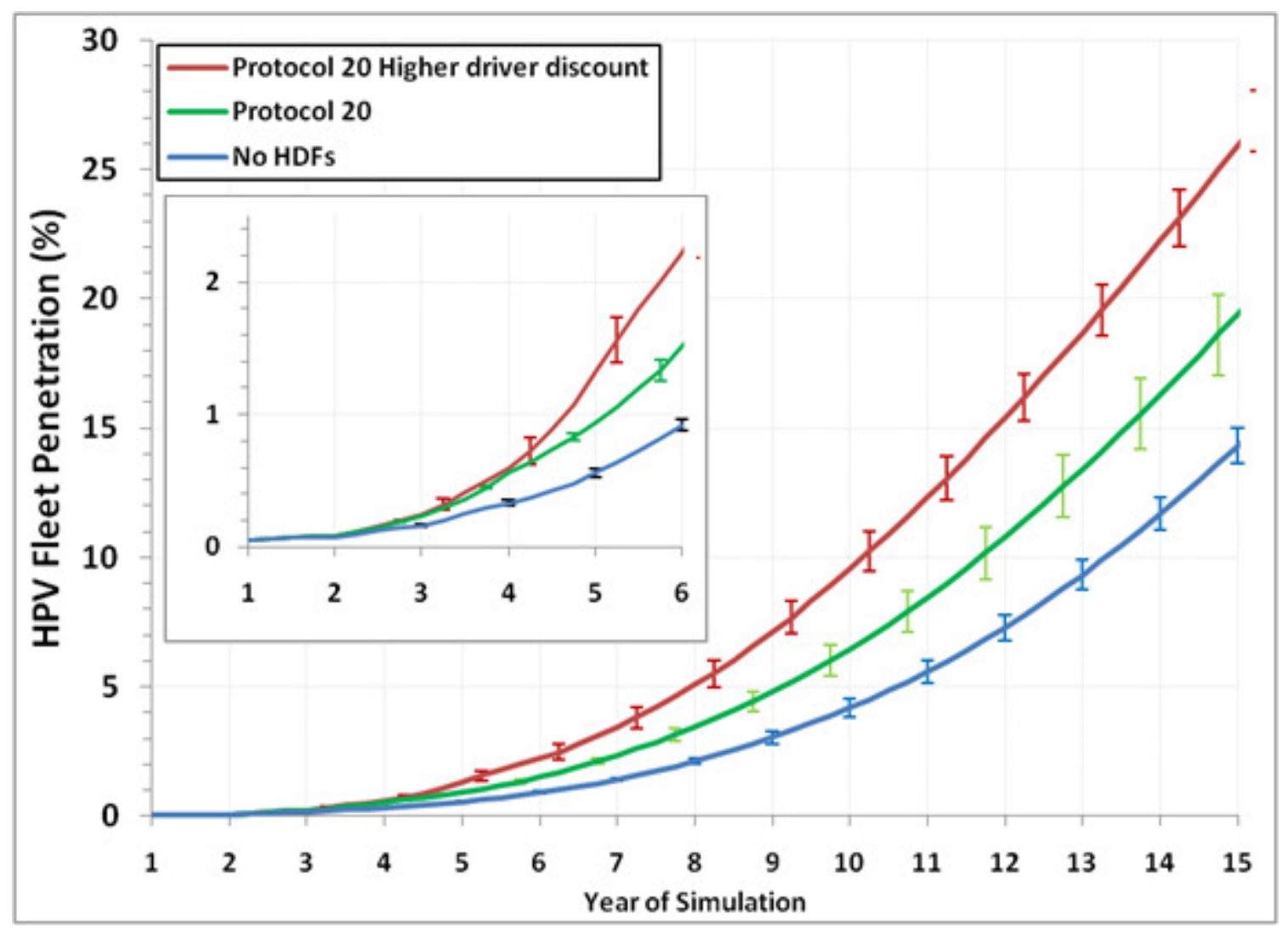

Figure 17: HPV Adoption Rate When Drivers Discount Past Experience More Heavily (Inset: Expanded vertical scale for early years showing little difference until years 4-5)

\subsubsection{HFS Investor Returns}

Because HDFs are directly competing with HFSs - at least, as much as they can with their limited capacity - one might expect that they might do so at the expense of the returns on HFS investments. This turns out not to be true. Figure 18 shows both the HFS investor agent's internal rate of return (IRR) and the net present value of his investment under two scenarios, one in which there are no HDFs, and another in which HDFs are sited under the reasonably aggressive Protocol 20. In both cases, the investor agent's IRR drops rapidly in the first few years as he rapidly builds new HFSs, then begins to increase after year 4 (the third year of investing). The IRR for the case in which HDFs are present remains higher thereafter for the full course of the 20-year simulation. The corresponding net present value (NPV), while marginally lower for the 
first 10 years, shows an even more dramatic difference in the later years, reaching a value almost twice that of the no-HDF case at the end of 20 years. This results from the more rapid penetration of HPVs caused by the HDFs, allowing the investor to build more HFSs, as shown in Figure 19.

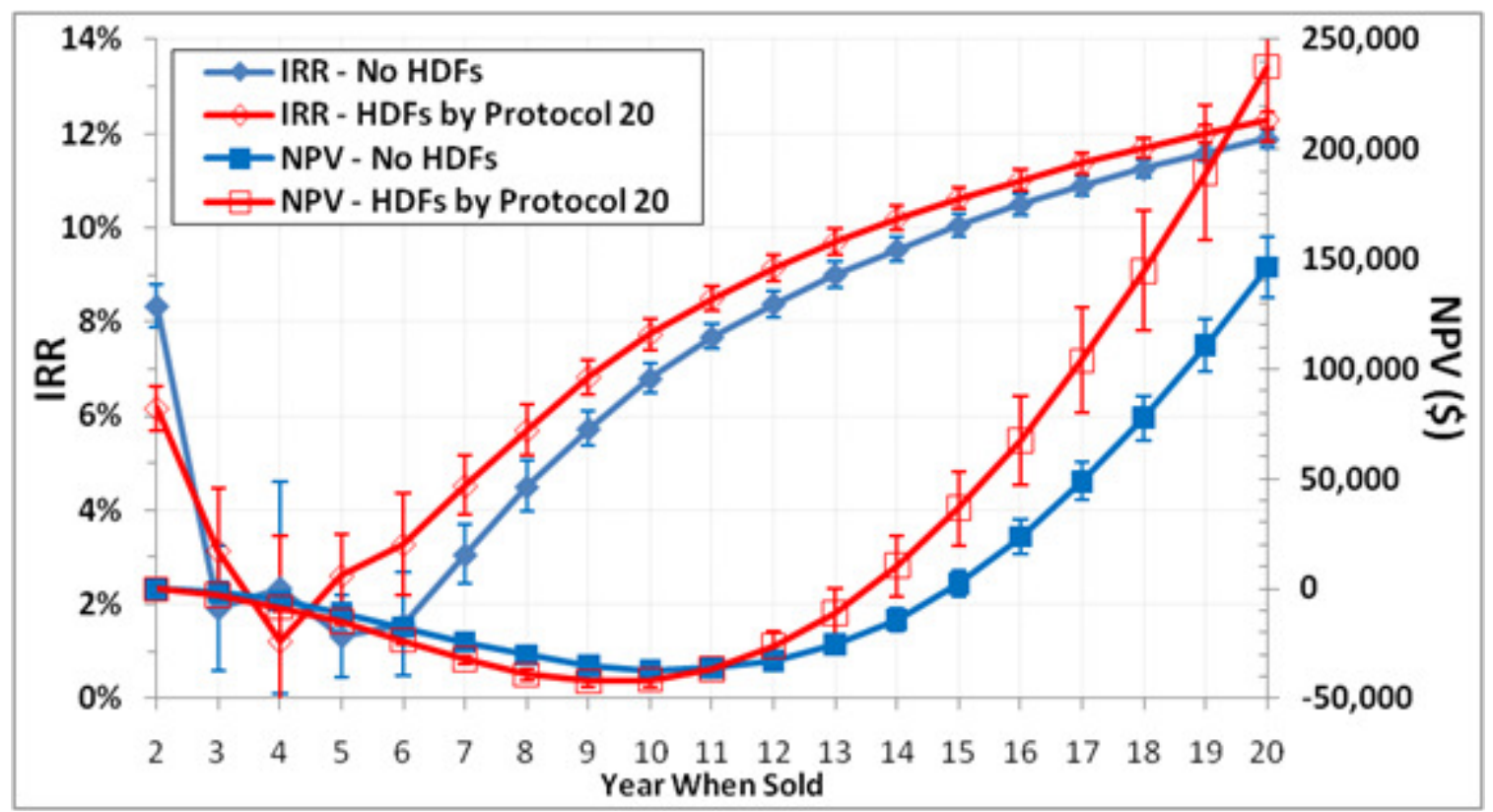

Figure 18: Investor IRRs and NPVs under Scenarios of No HDFs and "Protocol 20" Rollout of HDFs

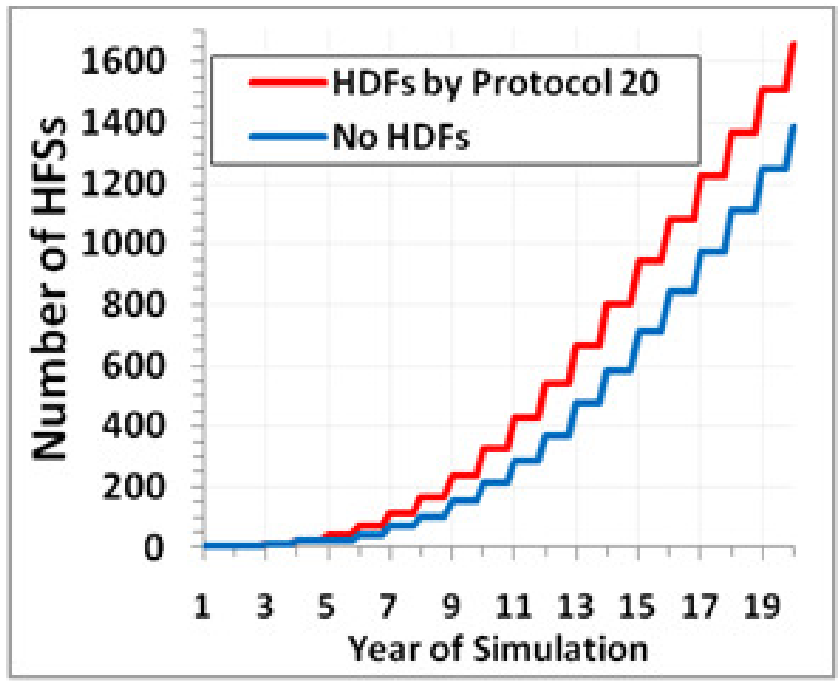

Figure 19: Number of Investor-built HFSs under the Two Scenarios in Figure 18 


\subsubsection{Geographic Results}

The observations we have presented thus far have focused primarily on the aggregate outcomes. When the ABMS technique is used, it is important to remember that these high-level results are produced by many low-level interactions among agents and the environment. The results of the model can also be investigated on an agent or geographic basis. In this instance, we start with the base case and compare a transition without any HDFs to one in which 75 HDFs come online over a course of 7 years, each having a capacity of $73 \mathrm{~kg}$ /day and selling fuel at a price of $\$ 3.00 / \mathrm{kg}$. In Figure 20, we plot the geographic distribution of per-capita hydrogen fuel consumption 2 years after the last of the HDFs has come on-line. Each cell is colored according to the difference in fuel purchases between the two cases from driver agents living in those cells. The grey indicates no change; the more intense green indicates higher fuel sales when HDFs are present; and the more intense red indicates lower sales. The cells outlined in yellow indicate the locations of HDFs.

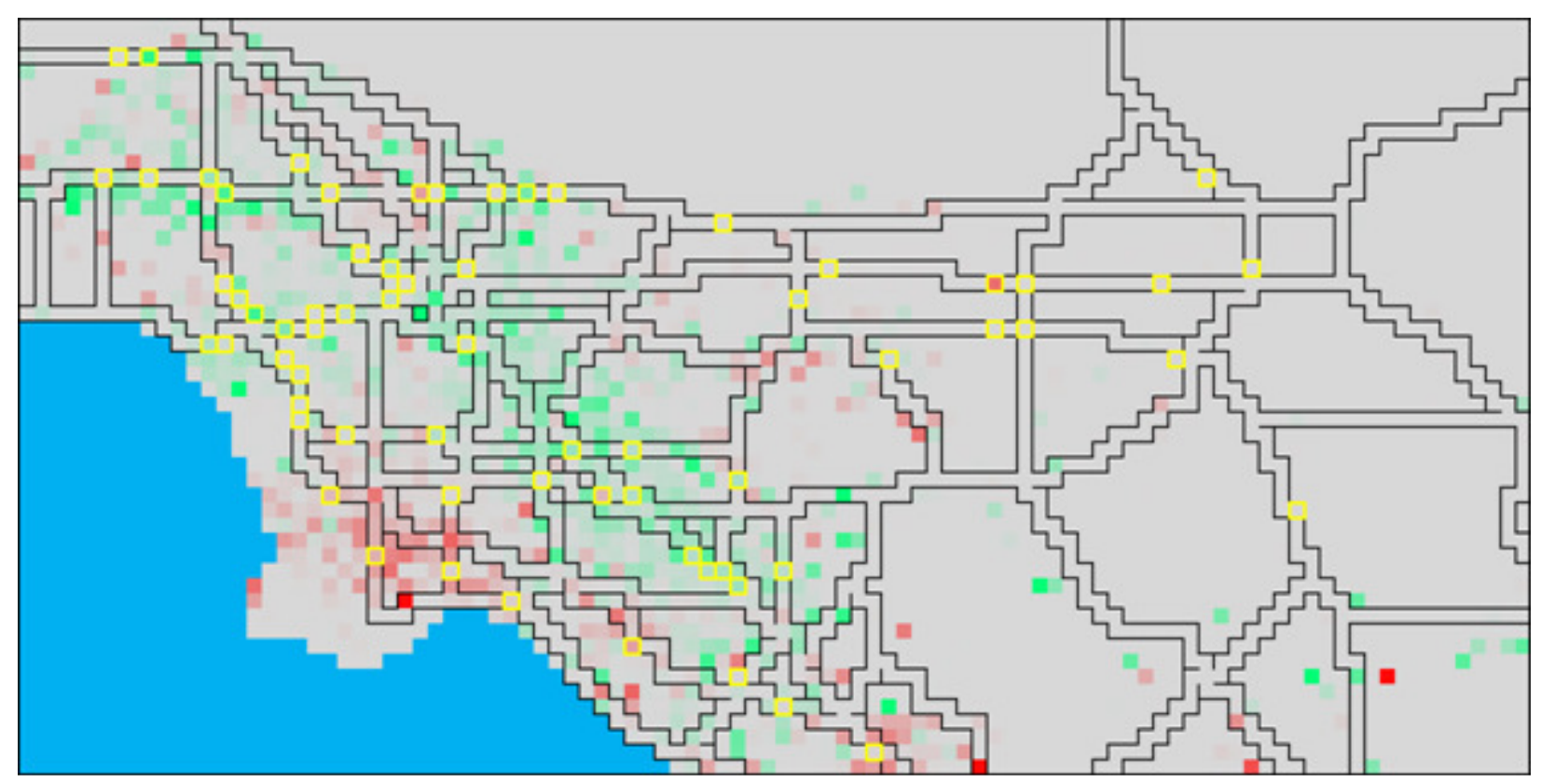

Figure 20: Difference in Household Hydrogen Fuel Consumption between a Case with and without HDFs

In general, we observe a good correlation between the presence of HDFs and an increase in fuel sales to driver agents living nearby, especially along the I-5 corridor (the major diagonal running northwest-southeast) and in the northwestern area of the grid. With some exceptions, the HDFs in the eastern area of the grid do not seem to have much of an effect on the driver agents there. In the Long Beach (southwestern) area, we interestingly see a relative decrease in fuel sales, even though there are HDFs present in that area. Those HDFs, however, are among the last to be established. Of course, the HDFs alone are not responsible for the differences we see between these two cases. The availability of HFSs primarily explains the differences. In the case with HDFs, the areas with a strong correlation of HDFs to increased fuel sales are among the first 
areas to have established HDFs. The presence of those HDFs leads drivers living in those areas to adopt HPVs more rapidly, and so the investor agent unsurprisingly focuses early development efforts in those areas, which leads to a positive feedback loop with more drivers adopting and more infrastructure developing. By the end, the HFS capacity difference in the Long Beach area reaches 22 percent between the two cases. 
Analysis of CHHP as a Bridge to a Hydrogen Transition

This page intentionally blank 


\section{Conclusions}

On the basis of the results presented in Section 5, we have identified a number of general conclusions about the efficacy of HDFs in aiding a transition from petroleum- to hydrogen-based transportation.

HDFs can indeed be helpful in accelerating a petroleum-to-hydrogen transition. Placed in areas where investors might not be willing to install large for-profit HFSs, HDFs can serve as a bridge until demand for hydrogen increases to the point at which larger stations are viable. For drivers to use them, however, HDFs must sell hydrogen at a price competitive with that of the larger HFSs. Because the marginal cost of producing hydrogen is expected to be higher for small capacities, HDFs may be uncompetitive without (possibly substantial) subsidies or other means to allow them to sell hydrogen at a price below the actual cost to produce and distribute it. For example, the loss could be taken on by the CHHP owners as a premium to be paid to obtain approval to install a CHHP facility. Fortunately, HDFs do not need to be subsidized for very long. In our model, installing HDFs only as demand for hydrogen increased was effective; then closing down all HDFs after 5 years into the transition made virtually no difference to the resultant accelerated outcome. Even installing much smaller numbers of HDFs in the second and third years only and then closing them at the end of the third year was helpful.

HDFs cannot do the job alone, of course. Their role in accelerating a transition is to get the ball rolling early on. Once the process is started, the rise in demand for both HPVs and hydrogen fuel will likely bring a number of positive feedbacks:

- The rise in fuel demand encourages investors to build more HFSs.

- The rise in sales volume allows OEMs to build and sell HPVs for a lower cost.

- Social networking influences have a greater positive effect on most drivers, encouraging them to purchase HPVs.

The importance of HDFs can vary with the circumstances under which they operate. In general, HDFs are most important when hydrogen adoption otherwise would be slow; if adoption is rapid without them, adding HDFs has little effect. Also, we find that HDFs have more effect when driver agents weight inconvenience and worry more heavily in their vehicle purchase decisions. In this situation HDFs are fulfilling exactly the purpose for which they were employed, namely reducing these negative factors by providing drivers more locations at which to refuel.

Our model also showed that low-capacity HDFs (73 kg/day) are effective, but many of them run out after selling all their available hydrogen. In such cases, increasing the capacity of the HDFs can be helpful.

Persuading drivers that the transition to hydrogen is "for real," such that they are willing to ignore past years' experience when hydrogen may not have been readily available, appears also to be quite effective in promoting a successful transition. 
Finally, an important conclusion is that HDFs, selling hydrogen at a subsidized price if necessary to be competitive with HFSs, do not "steal" sales from these privately-owned stations, at least over the long term. By allowing drivers to purchase hydrogen at places where HFSs are not available, HDFs increase the rate of HPV adoption so that private investors actually make more money over their investment horizon. 


\section{References}

1. RCF Economic and Financial Consulting, Inc. and Argonne National Laboratory, 2010, Hydrogen Production and Delivery Infrastructure as a Complex Adaptive System, Report Number DE-FG36-05GO15034.

2. Stephan, C.H., M. Mahalik, and T. Veselka, 2007, "Modeling the Transition to a Hydrogenbased Personal Transportation System," Proceedings of the Conference Frontiers in Transportation; Social Interactions, Amsterdam, Netherlands, October 14-16.

3. Mahalik, M., G. Conzelmann, C. Stephan, M. Mintz, and T. Veselka, 2007, "Transition to Hydrogen-based Transportation," Proceedings of the AGENT 2007 Conference on Complex Interaction and Social Emergence, Evanston, IL, November 15-17.

4. Mahalik, M., C. Stephan, G. Conzelmann, M. Mintz, G. Tolley, and D. Jones, 2009, "Modeling Investment Strategies in the Transition to a Hydrogen Transportation Economy," Proceedings of the NHA Conference and Hydrogen Expo, Columbia, SC, March 30-April 3.

5. Steward, D., M. Penev, G. Saur, and J. Zuboy, 2009, "Modeling Electricity, Heat, and Hydrogen Generation from Fuel Cell-Based Distributed Energy Systems," National Renewable Energy Laboratory, November, pp. 64-66.

6. Stephan, C.H., and J. Sullivan, 2004a, An Agent-based Hydrogen Vehicle/Infrastructure Model, Conference on Evolutionary Computation, Portland, OR, June 19-23.

7. Stephan, C.H., and J. Sullivan, 2004b, Growth of a Hydrogen Transportation Infrastructure, Agent 2004, Chicago, October 7-9.

8. Schelling, T.C., 1971, "Dynamic Models of Segregation," Journal of Mathematical Sociology 1, 143.

9. Schelling, T.C., 1978, Micromotives and Macrobehavior, Norton, New York, NY.

10. Schwoon, M., 2007, "A Tool to Optimize the Initial Distribution of Hydrogen Filling Stations," Transportation Research D 12, 70.

11. Keles, D., M. Wietschel, D. Möst, and O. Rentz, 2008, "Market Penetration of Fuel Cell Vehicles - Analysis Based on Agent Behaviour," International Journal of Hydrogen Energy $33,4444$.

12. McGuire, T., T. Roche, and A. Weinberger, 2008, "Analyzing Test Data from a Worldwide Fleet of Fuel Cell Vehicles at Daimler AG," The Mathworks News and Notes, October.

13. Davis, S., et al., 2010, Transportation Energy Data Book, ORNL-6985, Oak Ridge National Laboratory, Oak Ridge, TN. (Data are for 2001 MY vehicles.) 
14. Synovate Motoresearch, July 9, 2007 (private communication), See www.synovate.com/motoresearch/.

15. Oak Ridge National Laboratory, 2001, National Household Travel Survey, available at http://nhts.ornl.gov/.

16. DOE (U.S. Department of Energy), 2008, "Future (2025) Natural Gas Steam Reformer at Forecourt 1,500 kg/day," May 27, H2A 02D_Future_Forecourt_Hydrogen_Production_ from_Natural_Gas_(1,500_kg_per_day)_version_2.1.1-1.xlsb, spreadsheet, obtained from DOE H2A Production Analysis, available at www.hydrogen.energy.gov $/ \mathrm{h} 2 \mathrm{a}$

production.html, accessed February 17, 2009 (This document uses H2A default assumptions. Costs are in 2005 dollars.)

17. DOE, 2009, DOE H2A Standard Economic Assumptions, available at www.hydrogen.energy.gov/h2a_analysis.html, accessed February 17, 2009.

18. National Research Council, 2008, Transitions to Alternative Transportation Technologies: A Focus on Hydrogen, Committee on Assessment of Resource Needs for Fuel Cell and Hydrogen Technologies, ISBN 978-0-309-12101-9 (pdf).

19. Santini, D.J., and A.D. Vyas, 2005, Hybrid and Diesel Vehicle Introduction Influences in the Technology Adoption Life Cycle, 84th Annual Meeting of the Transportation Research Board, Paper No. 05-2434.

20. The National Hydrogen Association, 2010, Hydrogen Fueling Station Database, available at http://www.hydrogenassociation.org/general/fuelingSearch.asp, accessed October 2010. 


\section{Appendix A: Fit of Driver Agent Trip Length Distribution to NHTS Data}

This appendix outlines how driver agent trips are assigned. Driver agents are given a portfolio of 20 random trips, in addition to their single commute trip. Routes from their homes to these destinations are calculated in a pre-run routine. During the running of the simulation, driver agents choose randomly from this portfolio to make up their megatrip.

\section{A.1 Matching of Driver Trip Lengths to Survey Data}

It is impossible, within the present boundaries of the simulation, to have the driver agents' trips exactly match those of real drivers. The reasons for this are discussed in the following sections. Thus, the objective must be to provide the agents with trips such that their exposure to the hydrogen refueling infrastructure mirrors as closely as possible that of real drivers.

In the following discussion, we use the National Household Travel Survey (NHTS) [15], a national survey of household members, to determine all trips they took on a single day by any mode of transportation. The NHTS defines a "trip" as a movement from one point to another. However, in this discussion, we use the term "trip segment" for this movement, and "trip" or "round trip" to denote a movement from home to one or more destinations and a return home. Thus, a typical person's travel day might consist of a trip from home to destination A, from there to destination $\mathrm{B}$, then to destination $\mathrm{C}$, and back to home; then later, a trip from home to destination $\mathrm{D}$ and back, for a total of two trips comprising six trip segments.

To be usable for this simulation, the survey data required extensive manipulation. To obtain the travel of the household car(s), rather than the household members, we excluded all travel by other than personal vehicles (primarily cars, vans, SUVs, light trucks) and travel in which the household member was not the driver of the vehicle.

Next, the distances from all trip segments for a given vehicle were added to determine the total distance each vehicle drove during the day. From this total, the frequency distribution of daily mileages was obtained (Figure A.1). The NHTS provides weighting data reflecting the statistical significance of each trip. We found that including or excluding the weights had negligible influence on the mileage distributions.

While it is straightforward in the simulation to match the mileage distribution obtained by the method described above, limitations on both the current methodology and the NHTS data prevent exactly matching the travel characteristics. In the first place, trips by the simulation agents are always exactly two segments: from home to a single destination and back, whereas the NHTS data show trips varying from 1 to 22 segments (Figure A.2). 


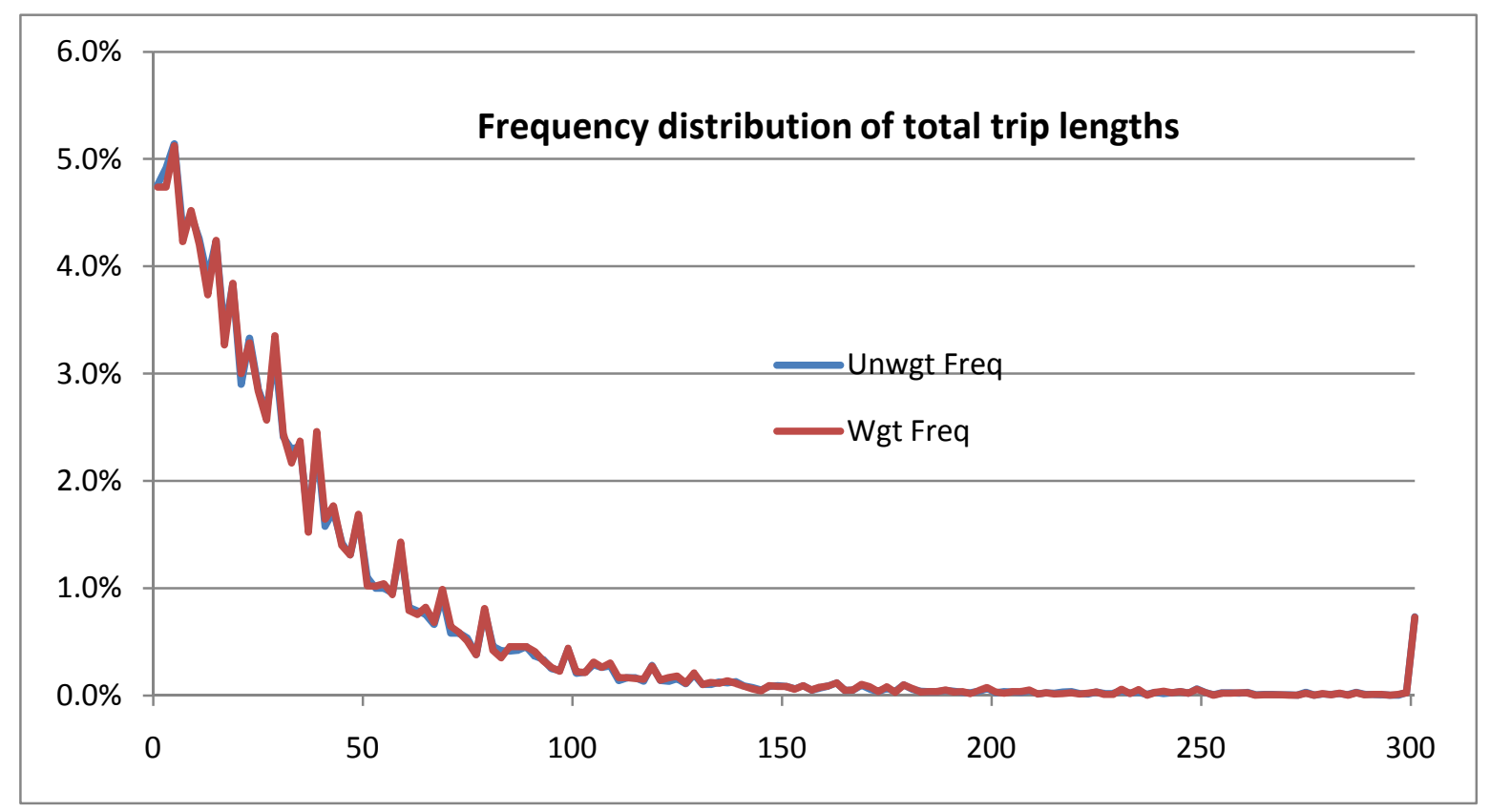

Figure A.1: NHTS Trip Length Frequency Distribution

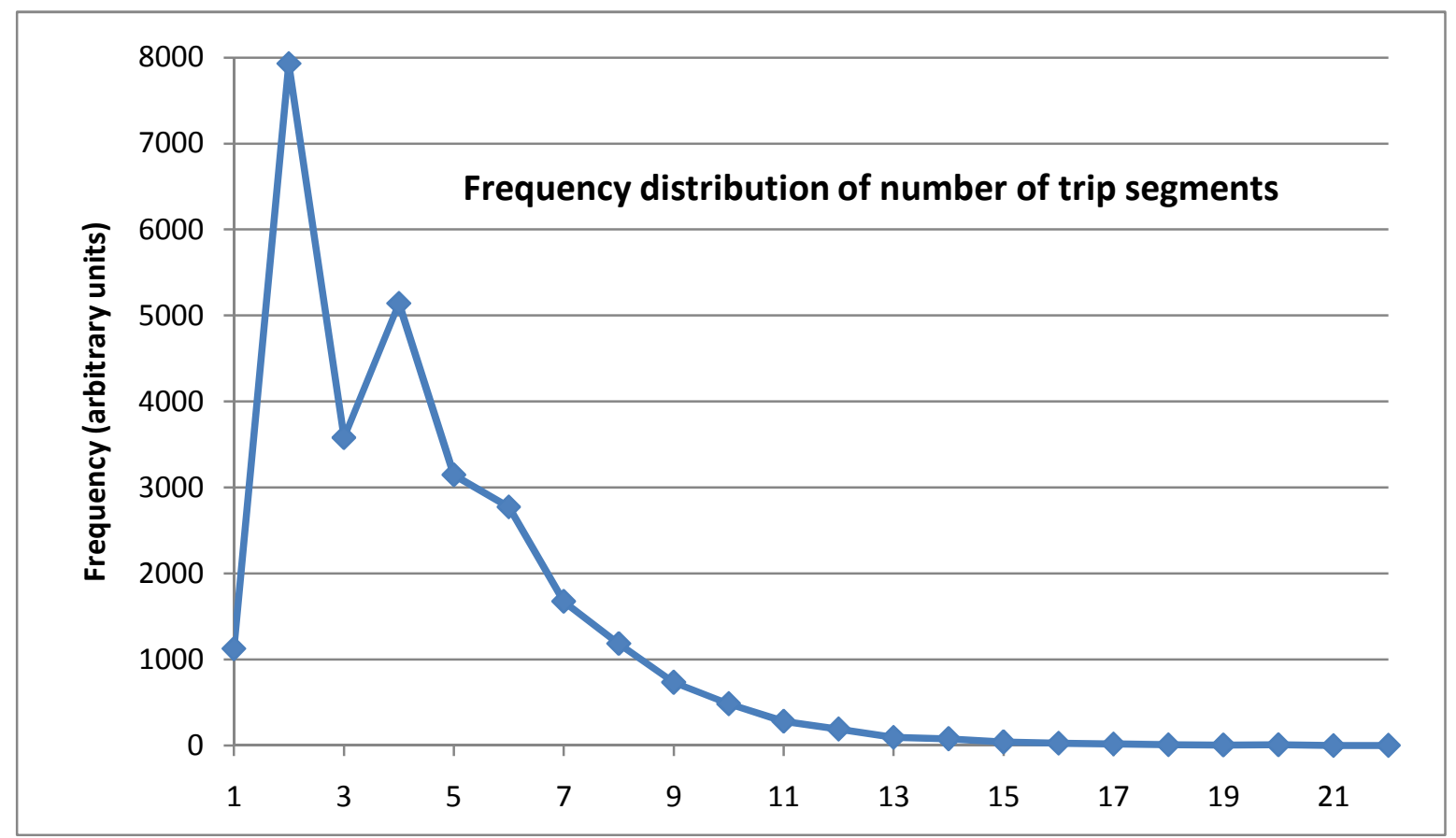

Figure A.2: NHTS Trip Segment Frequency Distribution

Further, the NHTS data do not include locational data, specifically, whether one or more of the destinations during the day was home. For example, for a one-segment trip, either the starting point or the ending point might be expected to be home; for a two- or three-segment trip, the starting point and final destination were very likely home. For a four- and higher-segment trip, 
there would be some probability that one or more of the intermediate destinations was home; that is, that the driver returned home after one trip and then he or another household member took the car out on a second or third trip later in the day.

Because of these limitations, agent drive characteristics cannot be fully matched to those of the survey. However, it is important that the most salient characteristics be matched as closely as possible. One such characteristic is the furthest point the driver reaches from home during the day. This is important because it determines how broad an area a single HFS can serve. If drivers stay close to home (driving a given number of miles in numerous short trips or longer ones composed of many segments to destinations near home), the presence of HFSs far from their homes will be of no benefit to them. Conversely, if drivers drive the same mileage in a single two-segment trip, they will more likely be able to take advantage of HFSs far from home. Of course, short trips can work to a driver's advantage, too - if there is an HFS near the driver's home and he never ventures far from home, he will never need to worry about the unavailability of fuel.

If our driver agents' trip lengths were calibrated to the calculated NHTS daily mileage, they would range much further from home in their two-segment trips than real drivers. Conversely, if their one-way-trip lengths matched the distribution of trip segment distances of the survey, they would clearly stay much closer to home than is realistic. The best approximation lies somewhere between these two extremes. In the absence of good data, estimates and simplifications were made. These are described in the sections that follow.

\section{A.1.1 Ratio of Trips to Trip Segments}

Here, two cases were examined. The first assumed that each vehicle in the survey made only a single trip from home and back, no matter how many segments it drove. The second assumed that all vehicles that drove four segments or fewer did so in a single trip, but vehicles that drove five segments or more made exactly two trips. In this case, we stipulated that one trip would comprise roughly two thirds of the trip segments ( 3 of 5, 4 of 6 or 7, 5 of 8, 6 of 9, etc.) and exactly two thirds of the total mileage, and the other trip would comprise the remainder. Only the second case is described here.

\section{A.1.2 Furthest Distance from Home as a Function of Number of Trip Segments}

In the case of a two-segment trip from home, the furthest distance from home is simply one half the total trip mileage (subject to a correction to be discussed). For multi-segment trips, that distance can range from one half the maximum single-segment distance to one half the total trip mileage. Multi-segment trips are considered to consist of a mixture of 90 percent closed random walks (starting point and ending point coincident) and 10 percent open random walks (ending at an arbitrary point generally different from the starting point). The reasoning behind this choice is as follows. From the NHTS data, we found that about 4 percent of all vehicles made a single one-segment trip, where (by definition) the trip was not "closed." Two- and three-segment trips were made by 40 percent of the vehicles, and we assume that almost all of these were closed. 
The closed or open nature of the remaining 56 percent of four- or more-segment trips cannot be determined. Thus, of the trips whose nature can be determined or estimated, roughly 10 percent of them were open.

To transform these results to ones that could be used in the simulation, we calculated the average straight-line distance from the starting point to the furthest point for closed and open random walks of unit distance and 2 to 22 segments. A random walk probably underestimates the furthest point distance, as drivers may attempt to order their intermediate stops to minimize the total driving distance (known in the literature as the traveling salesman problem), although in many cases this would not be possible. A typical example is a parent who drops a child off at day care on a trip in to work: while it might be shorter to drive first to the job location and then to the day care center, this obviously would not be feasible. To account for this partial ordering, furthest point distances, as calculated by the random walk routine, were increased by 10 percent for trips of four or more segments.

It should be noted that the furthest point, as calculated, is a straight-line distance. Driving on real roads to reach it would result in a longer driving distance. However, the ratio of driving to straight-line distances is probably roughly the same for the simulation and for real life, so no correction was made in either case. (For the simulation, the ratio is about 1.26:1.) Resulting data are shown in Figure A.3.

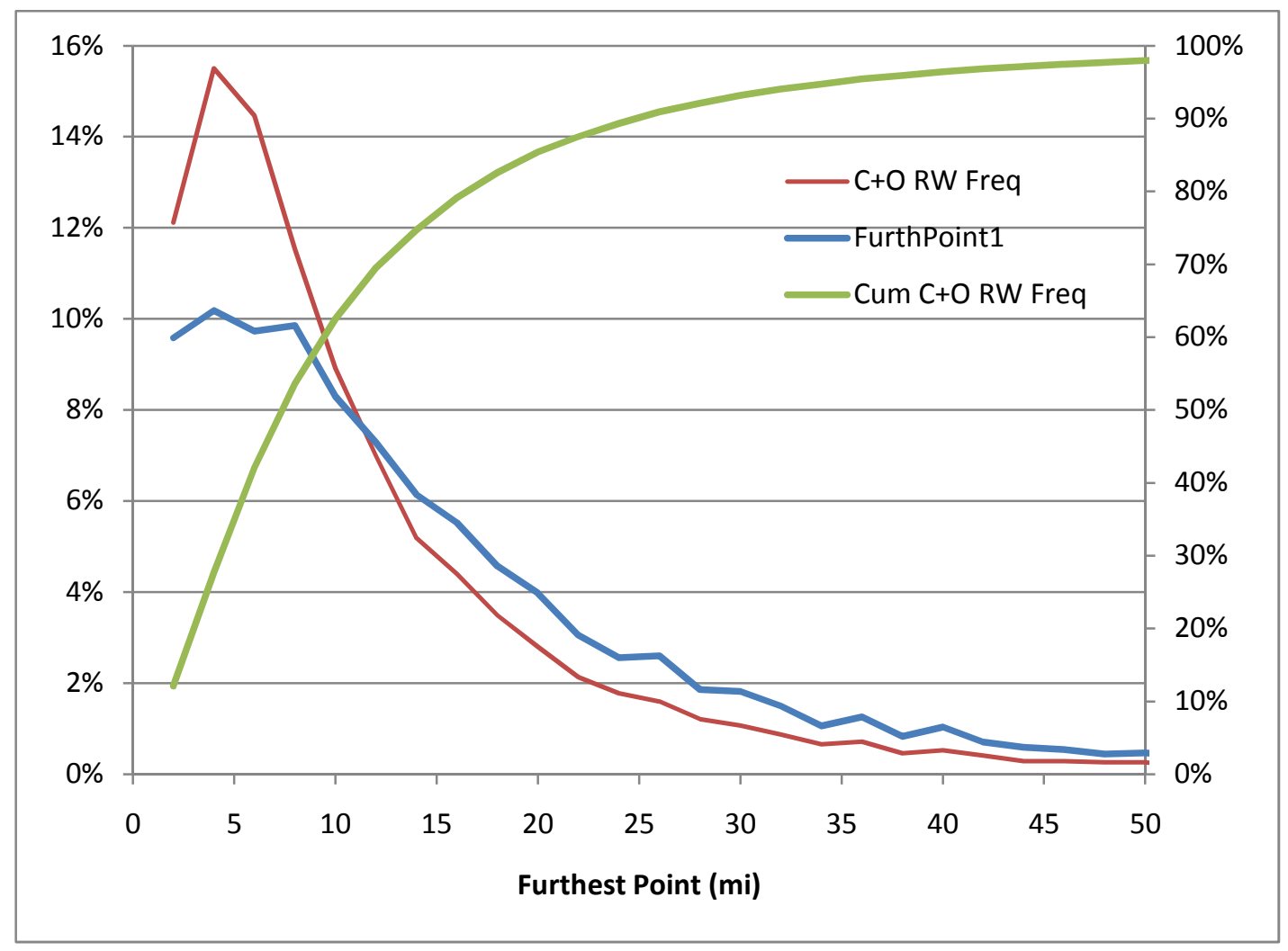

Figure A.3: Furthest Point from Home Frequency Distribution (Blue: Case 1 [single trip]. Red and Green: Case 2 [1 or two trips]) 


\section{A.2 Driver Trip Assignment}

On the basis of the calculations described in Section A.1, one objective in assigning driver trip destinations is to match the straight-line distances from the driver agents' homes to the straightline furthest point distribution calculated from the NHTS survey. There are additional objectives, to be discussed, for commute and random trips. Because of the simplifications made in the model, the multiple objectives are not necessarily mutually consistent. Thus, some tolerance had to be allowed in making trip assignments.

\section{A.2.1 Assignment of Job Locations}

A pre-run program makes the trip assignments. Driver agents are assigned home locations on the basis of cell population density, cell income level, and driver income level. The furthest-point frequency distribution described in Section A.1, aggregated into 2-mile bins from 2 to 150 miles, is read in from a file, as are the job density and salary density for each cell. Based on the driver agent population $(7,600)$, the frequency distribution is converted to a number distribution, and each furthest-point bin is allotted an appropriate number of trips. A lottery is constructed for each salary level, with each cell represented in the lottery as many times as it has jobs of a given salary level. (In general, a cell is represented with different frequencies in all three lotteries.) Drivers are chosen at random, with a salary-level weighting to be described later. A cell is chosen at random from the appropriate salary lottery, and the straight-line distance from the agent's home is calculated. The furthest-point bin containing that distance is checked, and if there is an allotment left, that cell is designated as the agent's work location, and the furthestpoint bin allotment is decremented by 1 . If the bin has no allotment left, another cell is chosen. The process is continued until a cell fitting the criteria is found. If no suitable cell is found after all cells of the appropriate salary level have been evaluated, the cell is chosen that results in the lowest furthest-point bin overfill ratio. The overfill ratio is defined as the ratio of the number of trips in a given furthest-point bin to the initial allotment of trips to that bin.

Because, statistically, a random cell choice is likely to result in a large straight-line distance from the agent's home and the frequency of long trips is low, the high furthest-point bins will be the first to be depleted. Thus, drivers chosen early in the lottery will be more likely to have long commutes than those chosen later. Because high-income workers in general might be expected to have longer commutes than low-income workers, the lottery is biased to favor high-income agents early in the draw and low-income ones later in the draw. Results are shown in Figure A.4. 


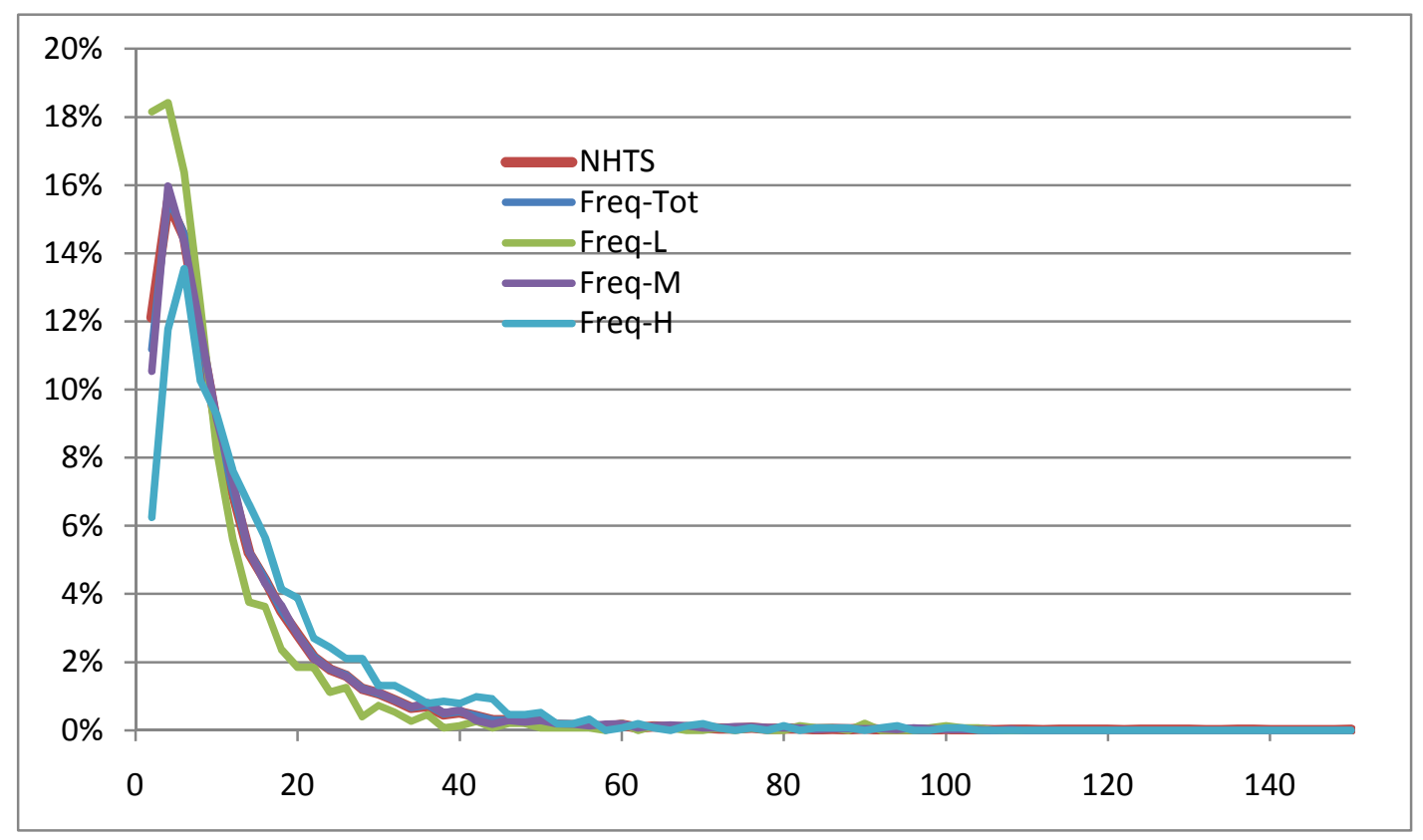

Figure A.4: Frequency Distribution of Distances of Agents' Work Locations from their Homes, Shown in Total and Separately for Agents of Low, Middle, and High Income, Compared to Calculated NHTS Furthest Point from Home Data

\section{A.2.2 Assignment of Random Trips}

In this procedure, an additional objective is to make the total number of trips to a given cell proportional to the population of that cell. The assumption here is that people make trips primarily to interact with other people or to visit institutions (shops, schools) whose density is proportional to population density. This is not entirely true. A shopping district or industrial center may attract many visitors (shoppers or workers) while having few residents. However, averaged over a 1-square-mile area, this is not a bad approximation. Most trips are to random cells chosen in this manner; some, however, are to predefined "attractor" cells that contain attractions such as sports stadiums, airports, concert venues, and theme parks.

A problem with performing the trip assignments as described is that the frequency of long trips is low, so with only 20 trips in each driver's portfolio, many drivers could end up with no long trips at all, a circumstance that would not be realistic. To overcome this challenge, we applied an equalization factor to the frequency-versus-trip-length distribution. The frequency of longdistance trips was increased to increase the likelihood that agents would be assigned at least one such trip. The factor by which the frequency of trips of that length was multiplied was stored along with the destination. During the simulation, random trips are chosen randomly, but with the probability of choosing a trip of a given distance weighted by the inverse factor. ${ }^{8} \mathrm{~A}$ procedure

8 An analogous equalization was applied by the Recording Industry Association of America to LP records, whereby the low-frequency components of the sound were deemphasized and the high-frequency components emphasized to mitigate the problem of high-amplitude excursions produced by low frequencies. An inverse equalization to recover the original sound was applied when the records were played back on a phonograph. 
similar to that used for commuter trips is used to assign random trips to agents. The 2-mile bins for furthest-point distance distribution (as equalized above) are populated based on 20 rather than 1 trip per driver. The total number of trips to be assigned (7,600 agents times 20 trips per agent less trips assigned to attractor cells) is calculated and distributed among cells both in the grid and in the extended area in proportion to cell population. In principle, then, each cell would be represented in a lottery in proportion to its population. In practice, however, the number of cells is so large (15,000 less zero-population cells) and the range of population densities so great ( 2 to $\sim 45,000$ per square mile) that a single lottery would not be practical. Instead, 16 population bins are assigned geometrically by powers of 2 (i.e., $2,4,8,16, \ldots 65536$ per square mile) and each cell is assigned to its appropriate bin. The bins (rather than the cells) are represented in a lottery according to the total population they represent. For example, for the main grid, the 265 cells with population $\leq 2$ (bin 2) constitute 0.004 percent of the population; the 496 cells with population between 8,192 and 16,384 (bin 14) constitute 37.9 percent; and the 11 cells with population between 32,768 and 65,536 (bin 16) constitute 16.6 percent (Figure A.5).

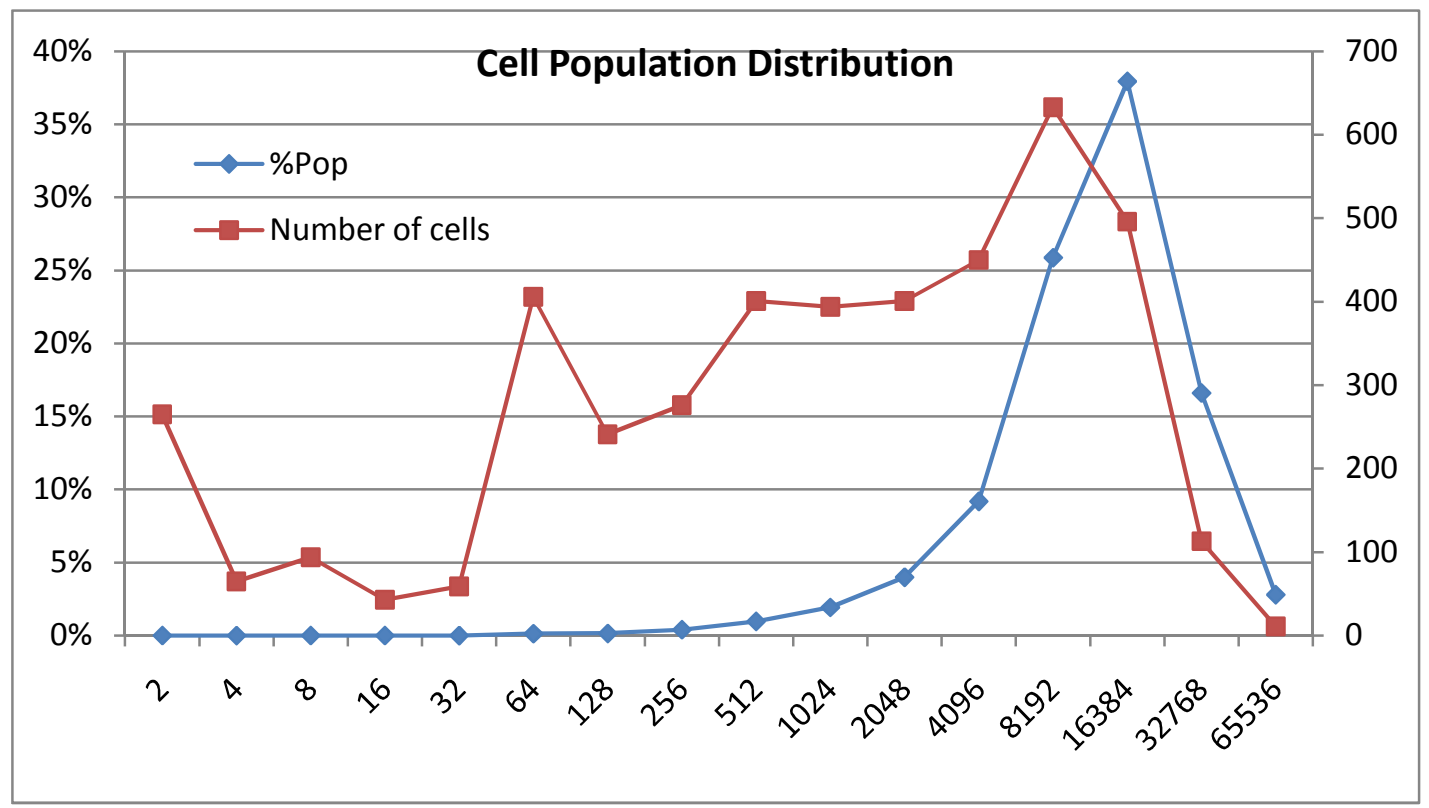

Figure A.5: Number of Cells of Different Population Densities (1 to 2 per square mile, 2+ to 4 per square mile, 4+ to 8 per square mile, etc.) and Contributions to Total Population

As an estimate for the population densities in the "extended region" bordering the modeled region, the average population density of the bordering cells (with non-zero-population density) on each of the north, south, east, and west edges of the grid is used for all "accessible" cells of the corresponding extended region.

In the assignment procedure, drivers are chosen randomly and assigned "attractor" destinations. Next, drivers are again picked randomly and given trip destinations of 1-2 miles and 2-4 miles until these distance bins are filled. For the rest of the trip assignments, the cell population lottery 
is used. A driver is picked randomly; a population bin is chosen randomly based on its representation in the lottery;, and then a cell in that bin is again picked (completely) randomly. As with the commuter trips, the distance from the agent's home is computed and checked against the allocation for that bin. If the bin is not depleted, that cell is assigned as the trip destination, and the bin is decremented by one. If the bin is depleted, another cell is chosen at random based on its population representation in the lottery, and its distance is again computed. The process is continued until a suitable cell is found. If no cell has been found after 5,000 tries, the cell producing the lowest furthest-point bin overflow ratio (as defined above) is chosen. The process continues until all agents have been assigned their full complement of 20 trips. Results are shown in Figure A.6.

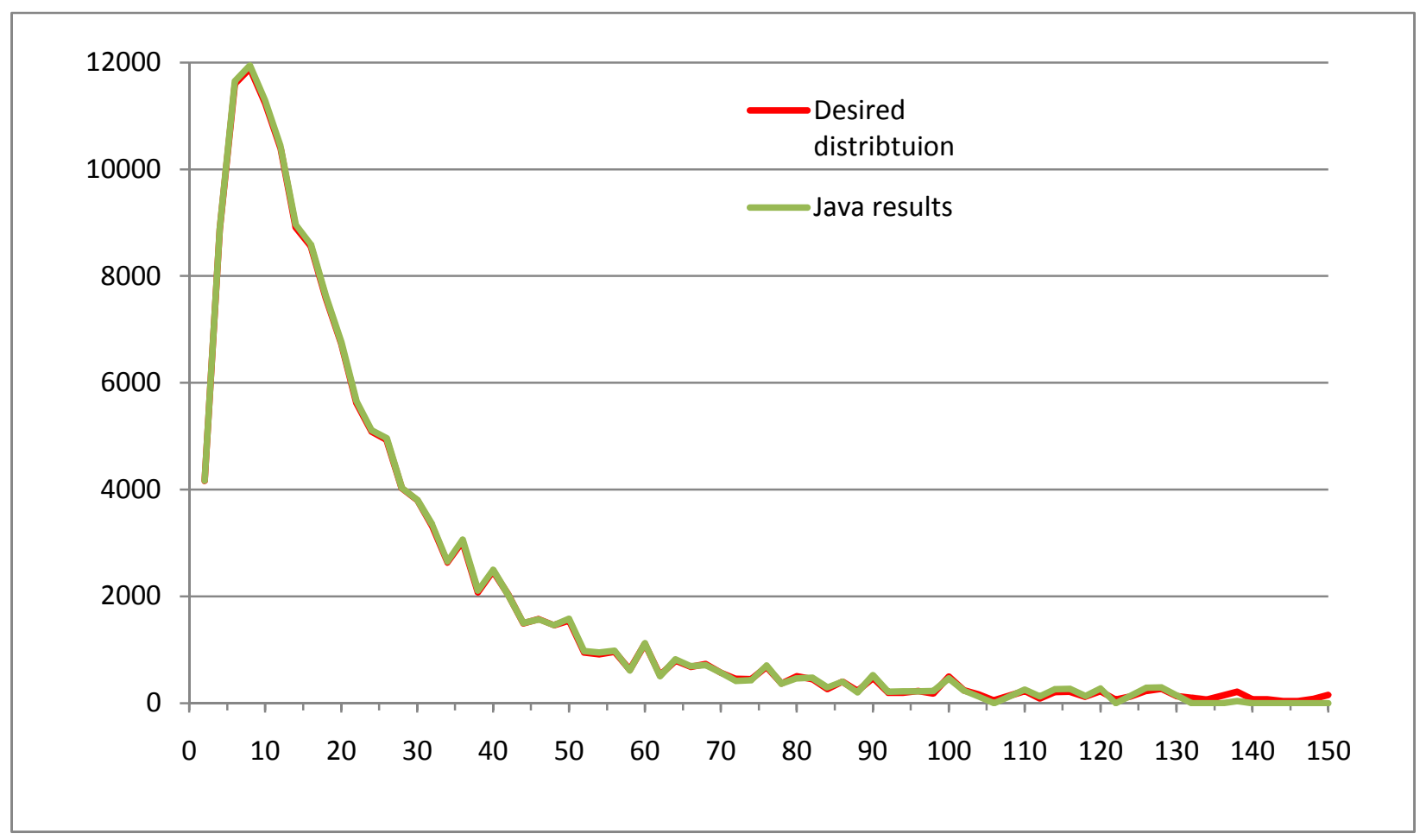

Figure A.6: Final Numbers of Random Trip Straight-Line, One-Way Distances (including Trips to Attractors) Average distance is $23.5 \mathrm{mi}$ (c.f. $36.7 \mathrm{mi}$ for the case where all daily driving was considered to be a single trip). 


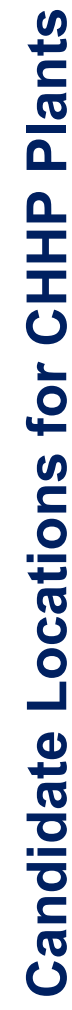

>

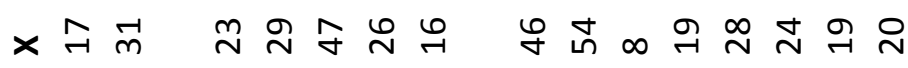
o

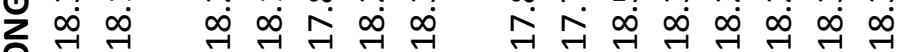

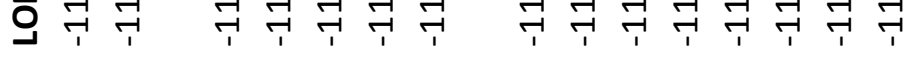

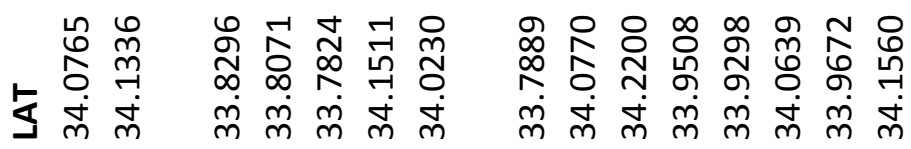

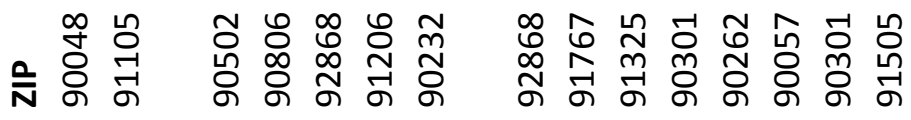

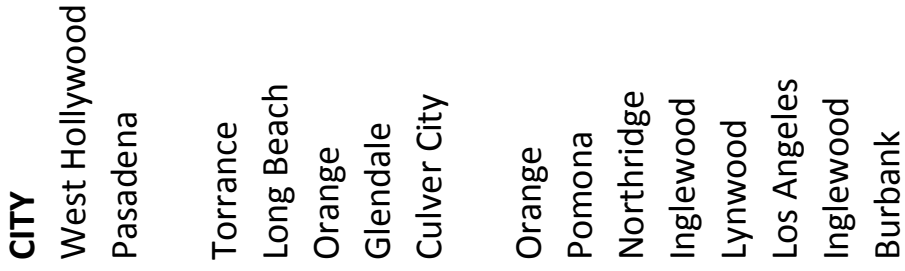

do

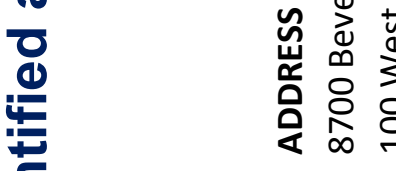

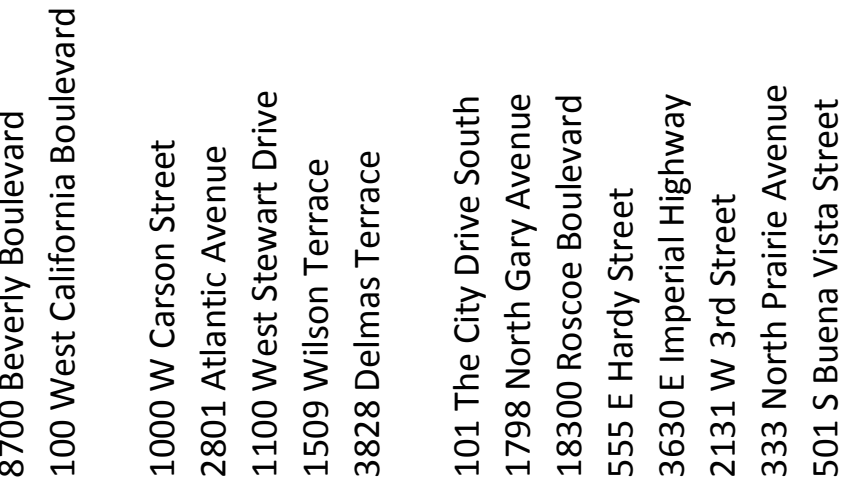

$\frac{10}{0}$

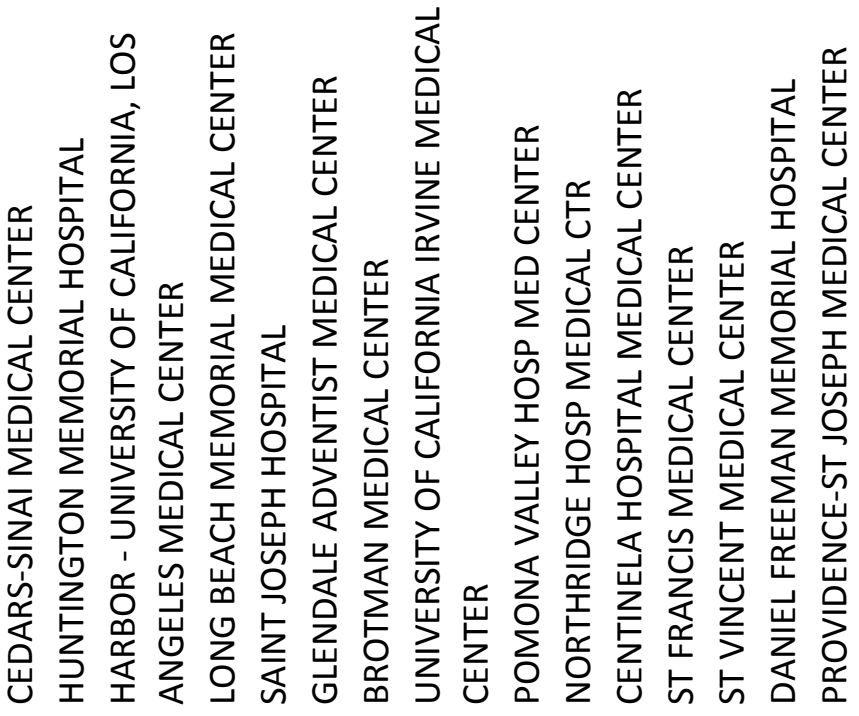


$>\stackrel{\infty}{\sim} \stackrel{\infty}{\sim}$ 곽 ×

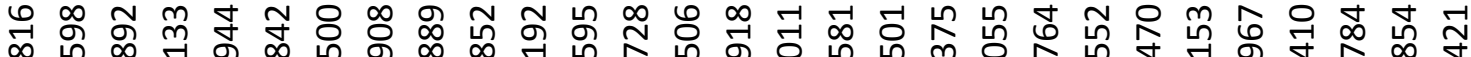
ט

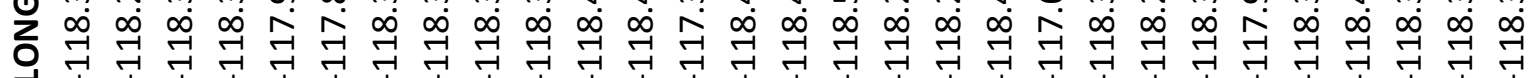

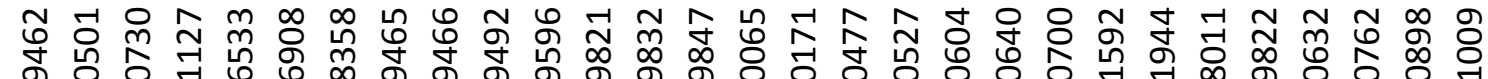

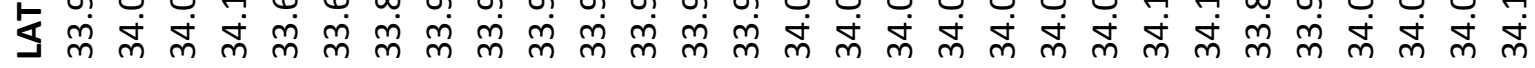

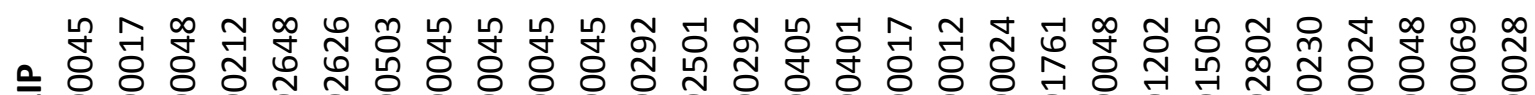

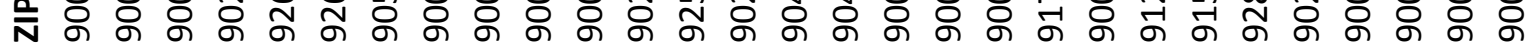
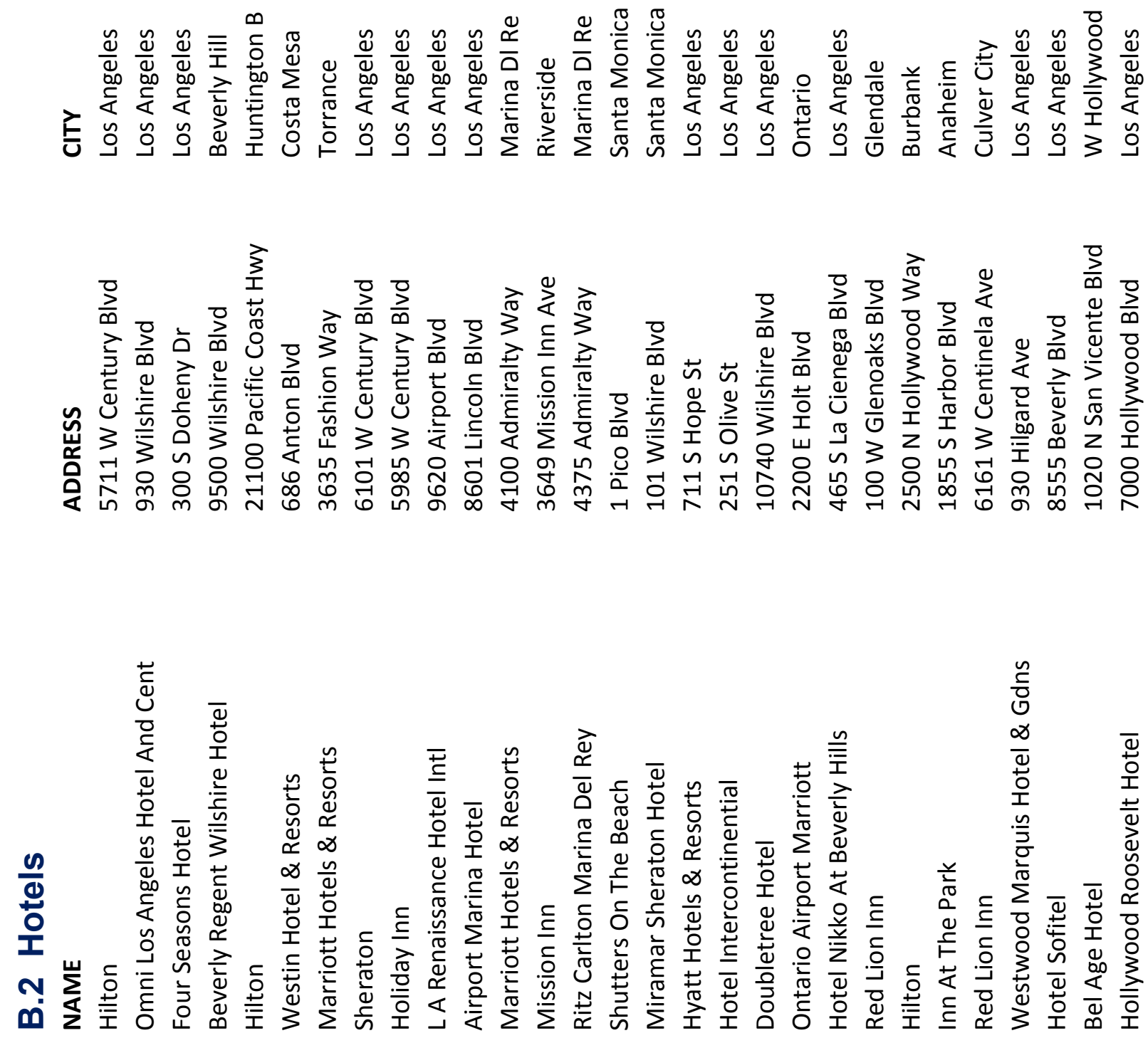


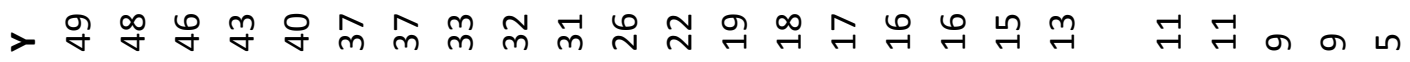

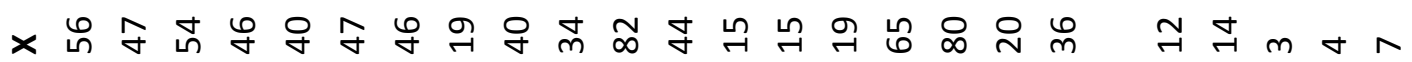

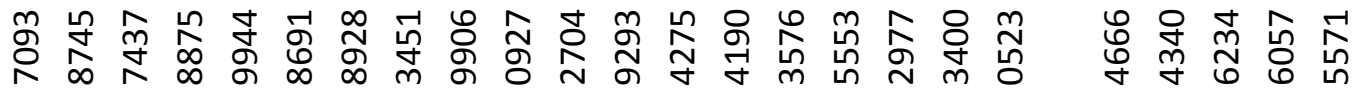

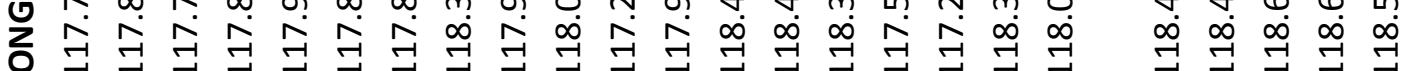

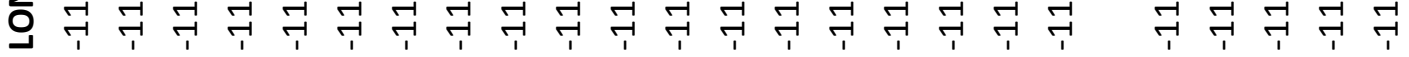

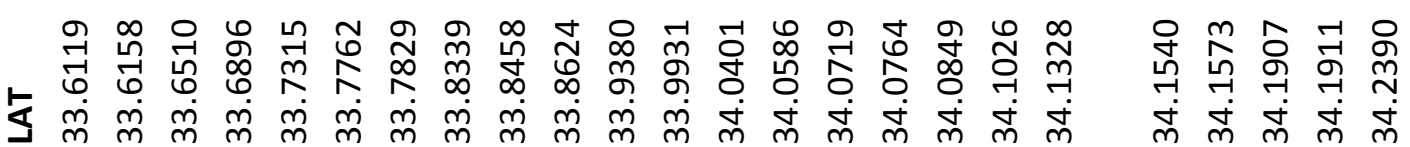

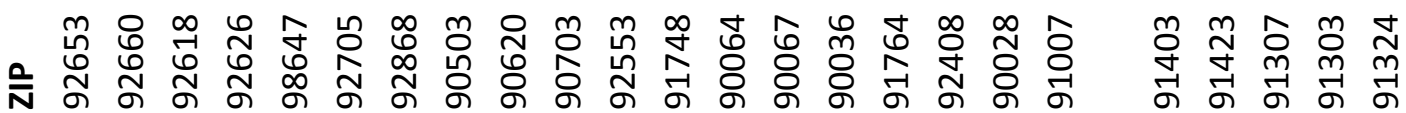

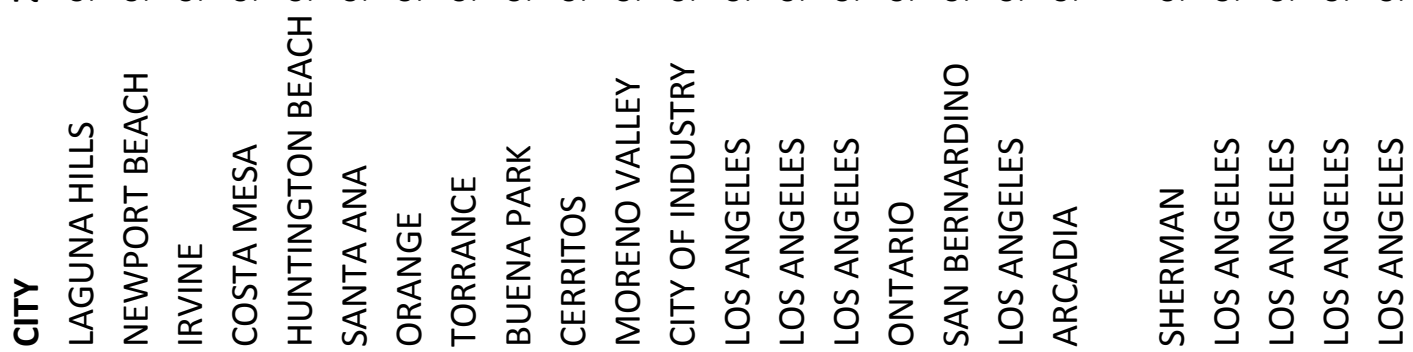

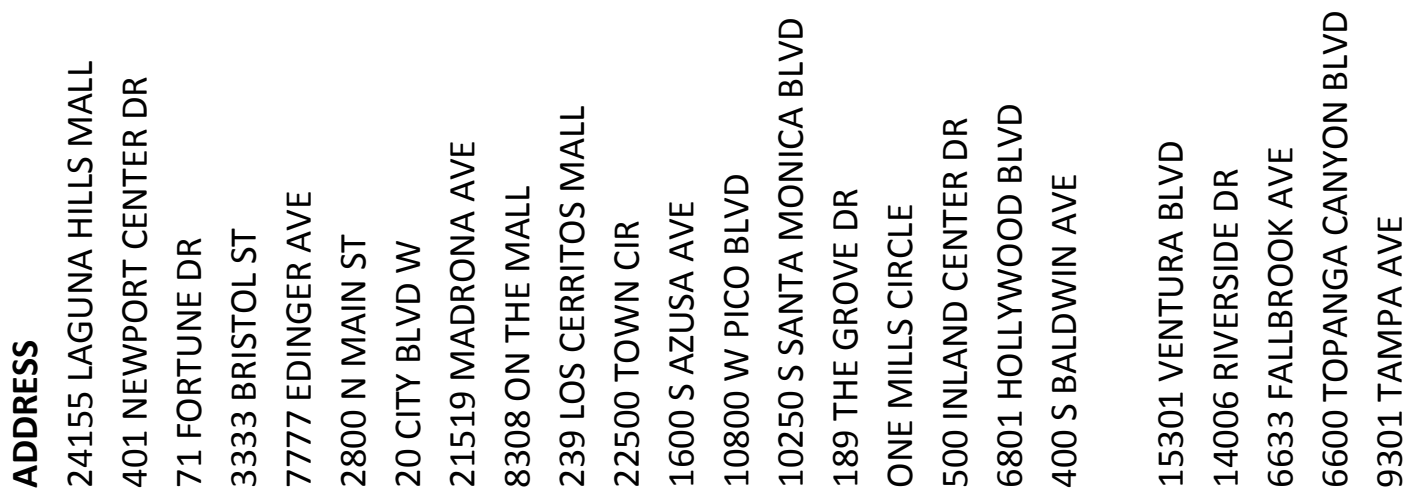

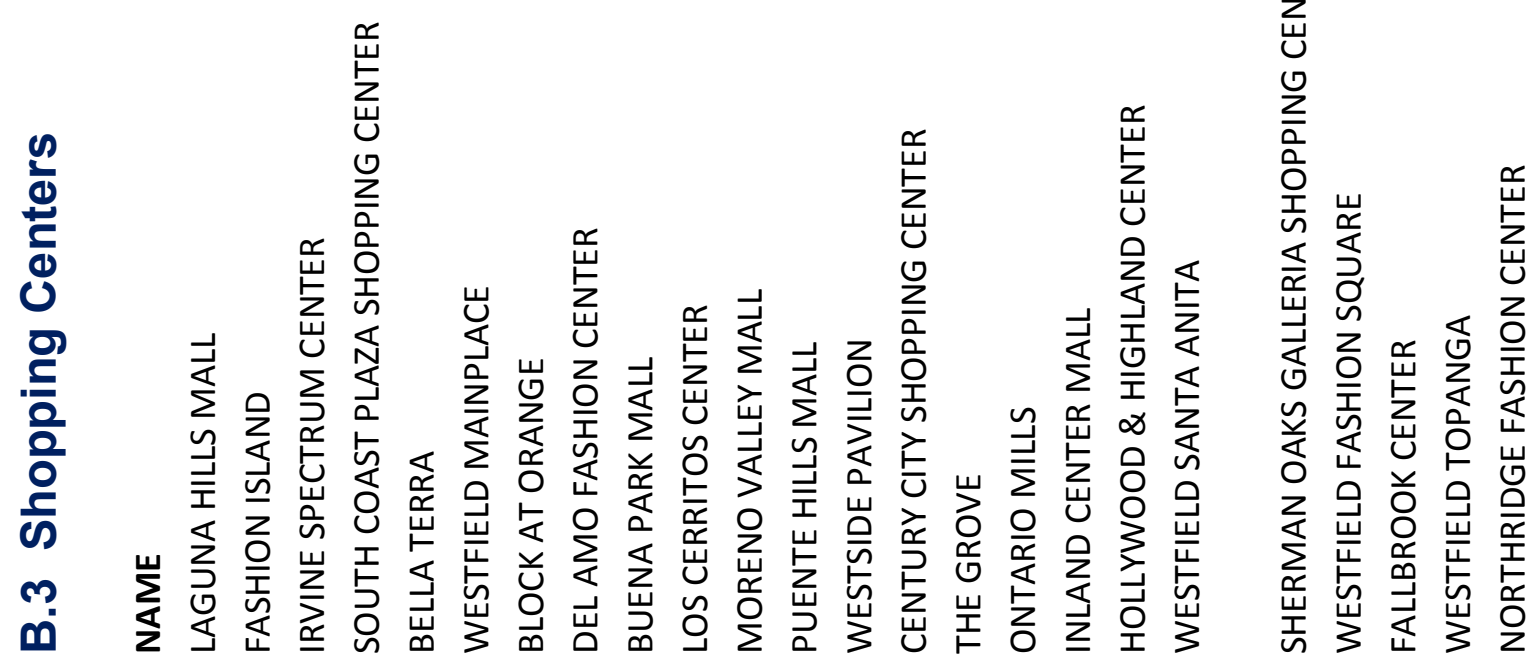


> × $⿻$ 寸

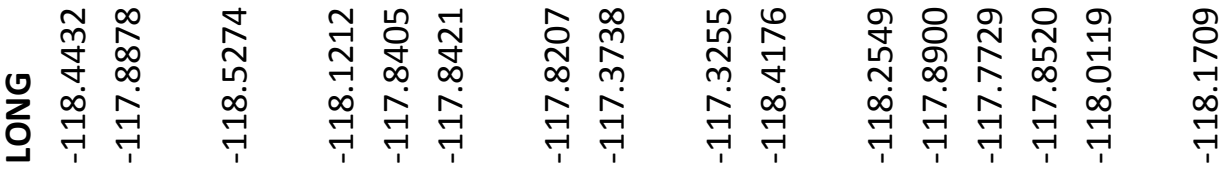

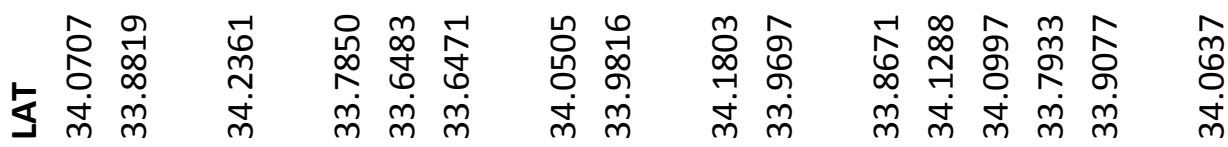

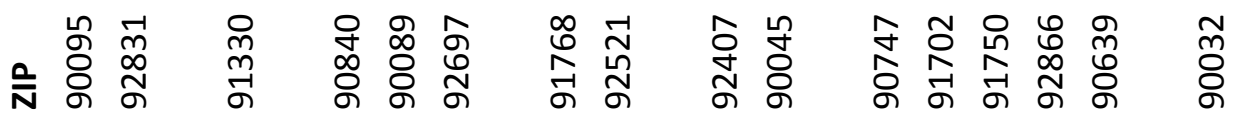

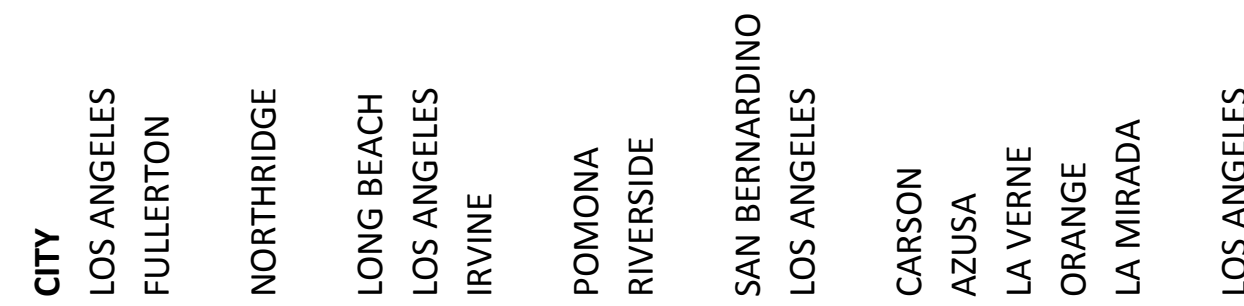

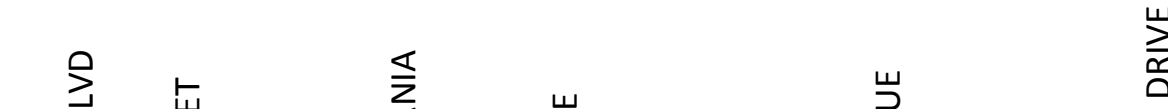

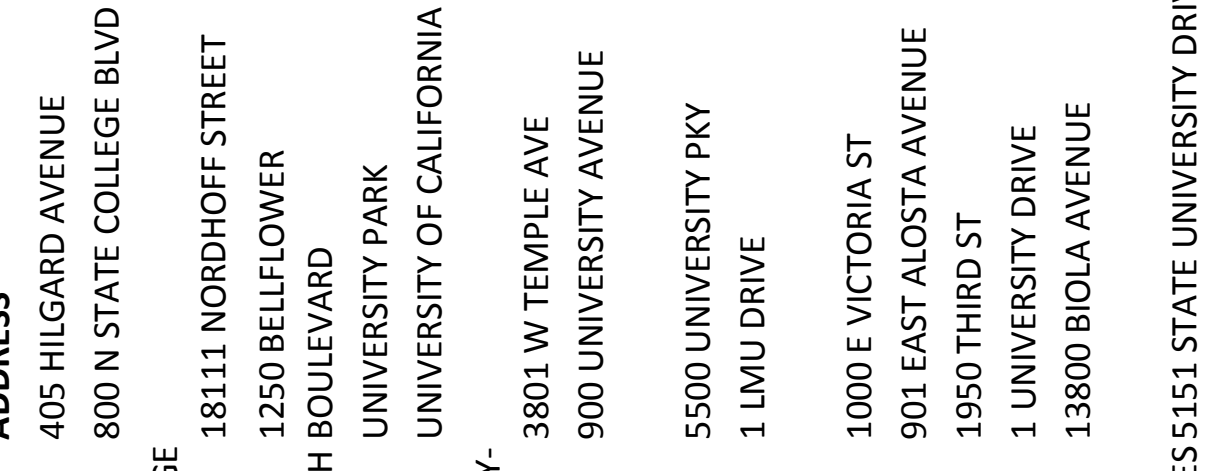

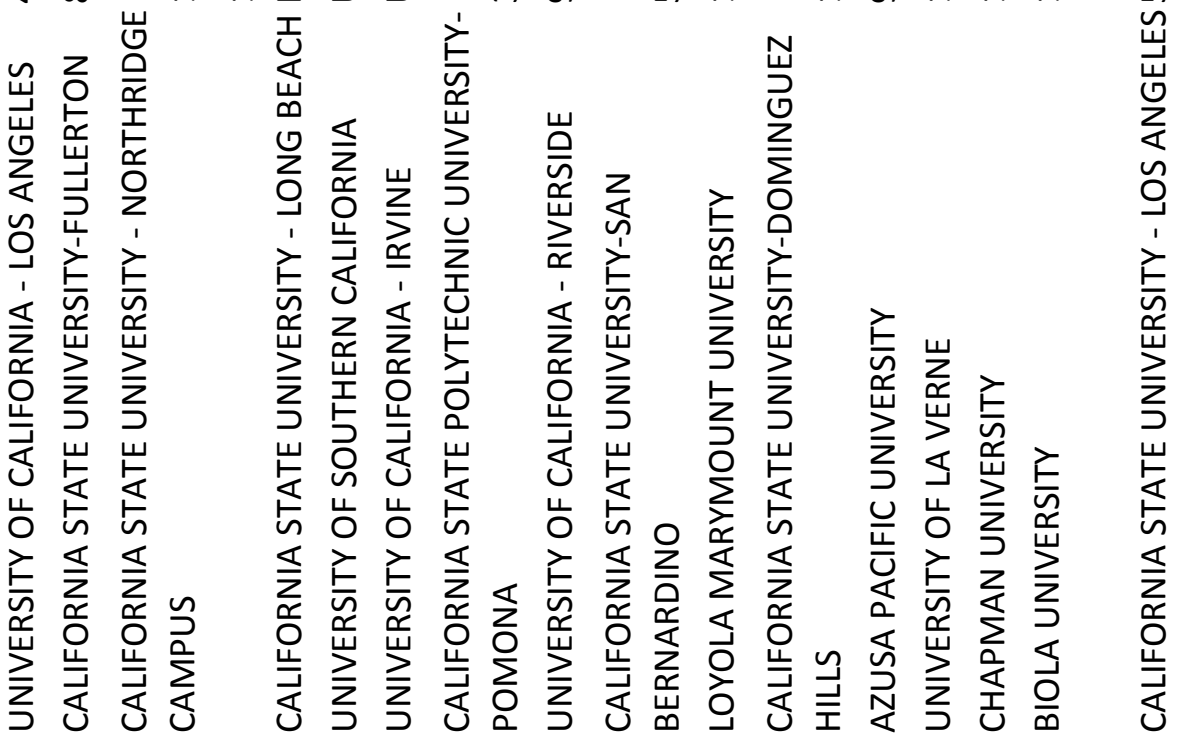




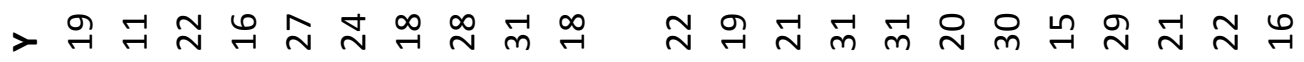

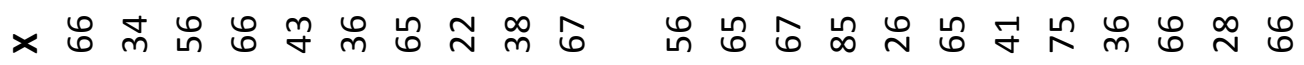

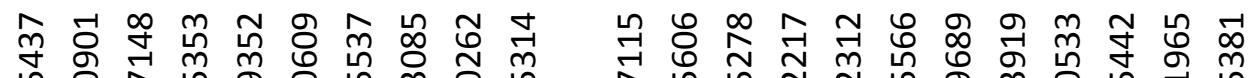

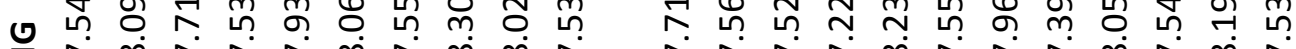

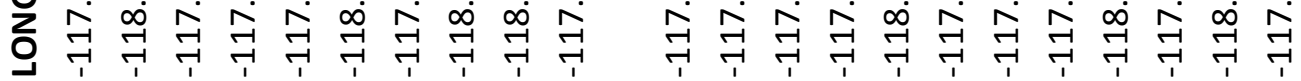

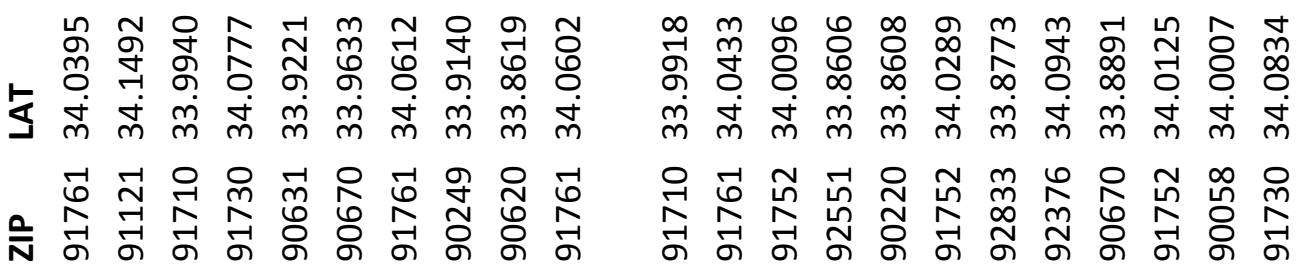

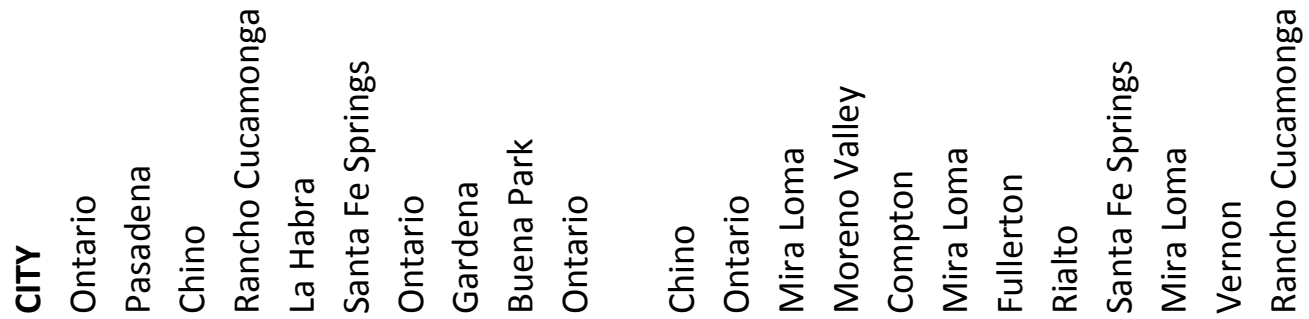

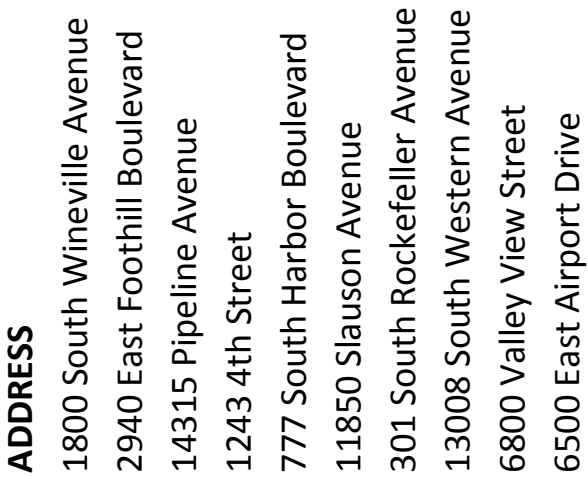
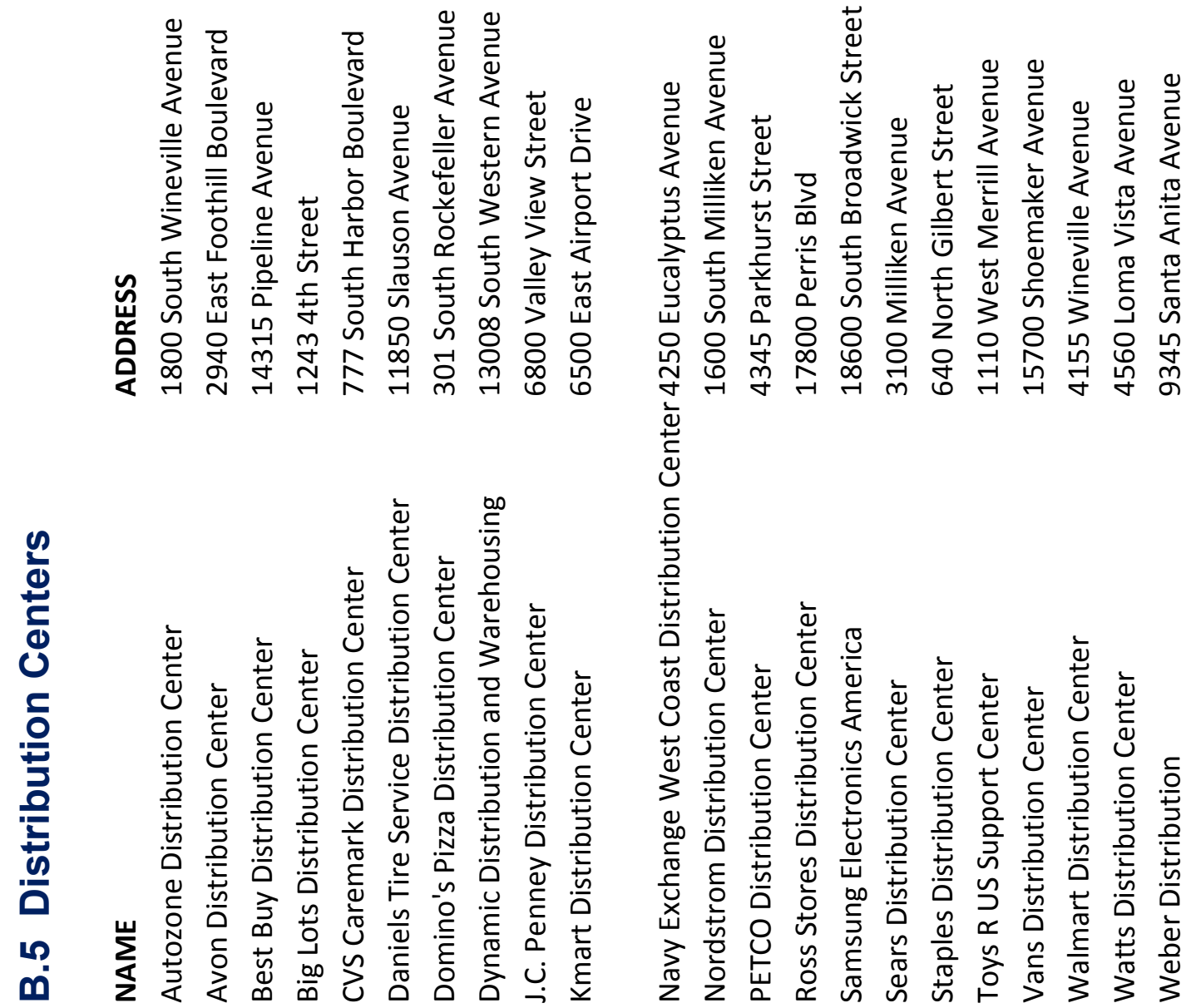


\section{Argonne}

Decision and Information Sciences Division

Argonne National Laboratory

9700 South Cass Avenue, Bldg. 221

Argonne, IL 60439-4844

www.anl.gov

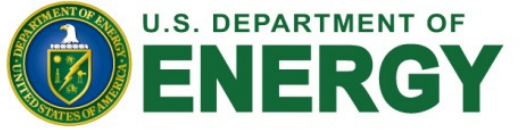

Argonne National Laboratory is a U.S. Department of Energy

laboratory managed by UChicago Argonne, LLC 